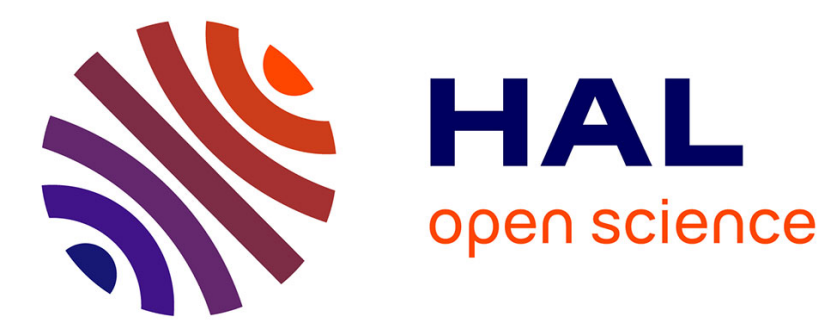

\title{
Dimensions of triangulated categories
}

Raphaël Rouquier

\section{- To cite this version:}

Raphaël Rouquier. Dimensions of triangulated categories. 2004. hal-00000698v3

\section{HAL Id: hal-00000698 \\ https://hal.science/hal-00000698v3}

Preprint submitted on 16 Sep 2004

HAL is a multi-disciplinary open access archive for the deposit and dissemination of scientific research documents, whether they are published or not. The documents may come from teaching and research institutions in France or abroad, or from public or private research centers.
L'archive ouverte pluridisciplinaire HAL, est destinée au dépôt et à la diffusion de documents scientifiques de niveau recherche, publiés ou non, émanant des établissements d'enseignement et de recherche français ou étrangers, des laboratoires publics ou privés. 


\title{
DIMENSIONS OF TRIANGULATED CATEGORIES
}

\author{
RAPHAËL ROUQUIER
}

\begin{abstract}
We define a dimension for a triangulated category. We prove a representability Theorem for a class of functors on finite dimensional triangulated categories. We study the dimension of the bounded derived category of an algebra or a scheme and we show in particular that the bounded derived category of coherent sheaves over a variety has a finite dimension. For a self-injective algebra, a lower bound for Auslander's representation dimension is given by the dimension of the stable category. We use this to compute the representation dimension of exterior algebras. This provides the first known examples of representation dimension $>3$. We deduce that the Loewy length of the group algebra over $\mathbf{F}_{2}$ of a finite group is strictly bounded below by the 2-rank of the group (a conjecture of Benson).
\end{abstract}

\section{CONTEnts}

1. Introduction 2

2. Notations and terminology 5

3. Dimension 5

$\begin{array}{lll}3.1 . & \text { Dimension for triangulated categories } & 5\end{array}$

$\begin{array}{lll}3.2 . & \text { Remarks on generation } & 7\end{array}$

\begin{tabular}{lll}
\hline 3.3. & Compact objects & 8
\end{tabular}

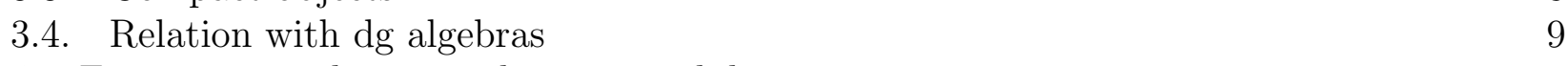

\begin{tabular}{llr}
\hline $4 . \quad$ Finiteness conditions and representability & 10
\end{tabular}

\begin{tabular}{|ll}
\hline $4.1 . \quad$ Finiteness for cohomological functors & 10
\end{tabular}

\begin{tabular}{|lll}
\hline 4.2. & Locally finitely presented functors & 15
\end{tabular}

\begin{tabular}{llr}
\hline 4.3. & Representability & 18
\end{tabular}

\begin{tabular}{lll}
\hline 4.4. & Finiteness for objects & 20
\end{tabular}

\begin{tabular}{lrl}
\hline $5 . \quad$ Localization & 21 \\
\hline 5.1. & 20 .
\end{tabular}

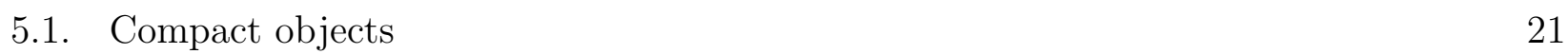

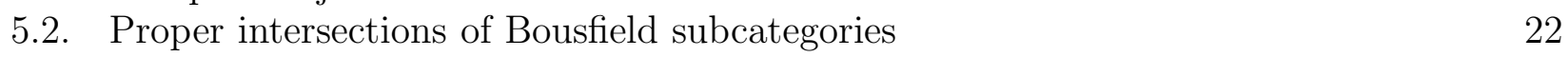

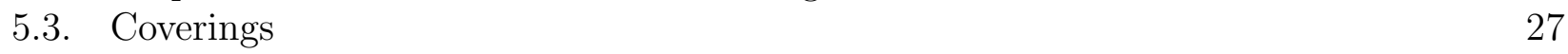

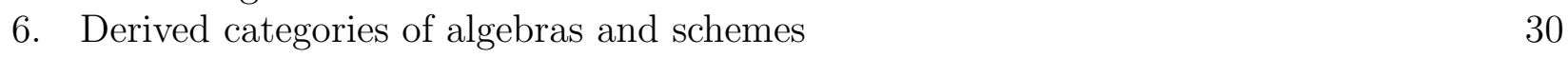

$\begin{array}{lll}6.1 . & \text { Algebras } & 30\end{array}$

$\begin{array}{lll}6.2 . & \text { Schemes } & 31\end{array}$

6.3. Compact objects in bounded derived categories 36

7. Dimension for derived categories of rings and schemes 37

\begin{tabular}{lll}
\hline 7.1. & Resolution of the diagonal & 37
\end{tabular}

\begin{tabular}{lll}
\hline 7.2. & Finite global dimension & 41
\end{tabular}

$\begin{array}{lll}7.3 . & \text { Nilpotent ideals } & 45\end{array}$

\begin{tabular}{lll}
\hline 7.4. & Finiteness for derived categories of coherent sheaves & 45
\end{tabular}

Date: Septembre 2004. 
8. Applications to finite dimensional algebras 49

8.1. Auslander's representation dimension 49

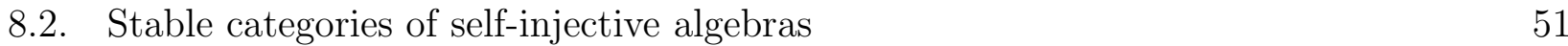

$\begin{array}{ll}\text { References } & 55\end{array}$

\section{INTRODUCTION}

In his 1971 Queen Mary College notes [A], Auslander introduced an invariant of finite dimensional algebras, the representation dimension. It was meant to measure how far an algebra is to having only finitely many classes of indecomposable modules. Whereas many upper bounds have been found for the representation dimension, lower bounds were missing. In particular, it wasn't known whether the representation dimension could be greater than 3 . A proof that all algebras have representation dimension at most 3 would have led for example to a solution of the finitistic dimension conjecture [IgTo].

We prove here that the representation dimension of the exterior algebra of a finite dimensional vector space is one plus the dimension of that vector space - in particular, the representation dimension can be arbitrarily large. Thus, the representation dimension is a useful invariant of finite dimensional algebras of infinite representation type, confirming the hope of Auslander. The case of algebras with infinite global dimension is particularly interesting.

As a consequence of our results, we prove the characteristic $p=2$ case of a conjecture of Benson asserting that the $p$-rank of a finite group is less than the Loewy length of its group algebra over a field of characteristic $p$.

Our approach to these problems is to define and study a "dimension" for triangulated categories. This is inspired by Bondal and Van den Bergh's work BoVdB and we generalize some of their main results. This leads us to look more generally at finiteness conditions for triangulated categories and their meaning in algebraic and geometric examples.

Our results also shed some light on properties of dg algebras related to geometry and might be viewed as requirements for non-commutative geometry. Let us give two examples.

- Given a projective scheme $X$ over a field, it is a classical fact that there exists a dg algebra $A$ with $D(A) \simeq D(X)$ : going to the dg world, $X$ "becomes affine". Given any such dg algebra $A$, we show that $\operatorname{dg}$ - $A$-modules with finite dimensional total cohomology admit "resolutions" (Remark 6.14), a strong condition on a dg algebra.

- Given a quasi-projective scheme $X$ over a perfect field, we show that there is a dg algebra $A$ with $A$-perf $\simeq D^{b}(X$-coh). Furthermore, for any such $A, A$-perf has finite dimension, a property which might be viewed as some kind of homological regularity for $A$ (when $A=A_{0}$ is noetherian and the differential vanishes, then $\operatorname{dim} A$-perf $<\infty$ if and only if gldim $A<\infty)$ : going to the $\operatorname{dg}$ world, $X$ "becomes regular". Note that this notion is weaker that smoothness of a $\operatorname{dg}$ algebra $A=$ perfection of $A$ as an $(A, A)$-dg bimodule.

More generally, let $\mathcal{T}$ be the bounded derived category of finitely generated modules over an artinian ring or over a noetherian ring with finite global dimension, or the bounded derived category of coherent sheaves over a separated scheme of finite type over a perfect field, or a 
quotient of any of these categories. Then, $\operatorname{dim} \mathcal{T}<\infty$, i.e., $\mathcal{T}$ is equivalent to $A$-perf, where $A$ is a dg algebra which is "homologically regular".

Let us review the content of the chapters. Chapters $\oint$ B 5 deal with "abstract" triangulated categories, whereas chapters $\$ 6$-8 deal with derived categories of rings and schemes (quasicompact, quasi-separated), and stable categories of self-injective finite dimensional algebras.

In a first part $\$ 3$, we review various types of generation of triangulated categories and we define a dimension for triangulated categories. This is the minimum number of cones needed to build any object (up to a summand) from finite sums of shifts of a given object. Note that we introduce and use later the notion of compactness of objects for triangulated categories that do not admit arbitrary direct sums.

We consider various finiteness conditions for cohomological functors on a triangulated category in $\$ 4.1$ and we derive some stability properties of these classes of functors. We define in particular locally finitely presented functors. On an Ext-finite triangulated category, they include locally finite functors. The crucial property of locally finitely presented functors is that they can be "approximated" by representable functors $(\$ 4.2$ and in particular Proposition 4.15). This leads, in $\$ 4.3$, to representability Theorems for locally finitely presented functors, generalizing Brown-Neeman's representability Theorem for "big" triangulated categories (cocomplete, generated by a set of compact objects) as well as Bondal-Van den Bergh's Theorem for "small" triangulated categories (Ext-finite, with finite dimension).

In $\$ 4.4$, we consider properties of objects $C$ related to properties of the functor $\operatorname{Hom}(-, C)$ restricted to compact objects. Later (Corollary 6.4 and Proposition 6.12), we determine the corresponding categories for derived categories of noetherian algebras or schemes : the cohomologically locally finitely presented objects are the complexes with bounded and finite type cohomology.

Part $\$$ 国 develops a formalism for coverings of triangulated categories mimicking the coverings of schemes by open subschemes. More precisely, we consider Bousfield subcategories and we introduce a notion of proper intersection of two Bousfield subcategories ( $\$ 5.2 .3)$ and we study properties of families of Bousfield subcategories intersecting properly. We obtain for example Mayer-Vietoris triangles (Proposition 5.10). The main part is $\$ \mathbf{5 . 3}$, where we consider compactness. We show that compactness is a "local" property (Corollary 5.12) - this sheds some light on the compact $=$ perfect property for derived categories of schemes. We also explain how to construct a generating set of compact objects from local data (Theorem 5.15). It is fairly quick to prove the existence of a compact generator for the derived categories of schemes from this (cf Theorem 6.8 for a version with supports).

Part $\S 6$ is a study of various classes of objects in derived categories of algebras and schemes. The main section $\$ 6.2$ considers complexes of $\mathcal{O}$-modules with quasi-coherent cohomology on a quasi-compact and quasi-separated scheme. We show how the length of the cohomology (sheaves) of a complex is related to the non-zero shifted groups of morphisms from a fixed compact generator to the complex (Proposition 6.9). We give a characterization of pseudocoherent complexes in triangulated terms (Proposition 6.10) : they are the objects whose cohomology can be "approximated" by compact objects — such a result is classical in the presence of an ample family of line bundles. 
In $\$ 6.3$, we show that for noetherian rings or noetherian separated schemes, the compact objects of the bounded derived category are the objects with finite type cohomology. This gives a descent principle.

In $\S$, we analyze the dimension of derived categories in algebra and geometry. In $\$ 7.1$, we use resolution of the diagonal methods. We show that for $A$ a finite dimensional algebra or a commutative algebra essentially of finite type over a perfect field, then $\operatorname{dim} D^{b}(A$-mod $) \leq$ gldim $A$ (Proposition 7.4). For a smooth quasi-projective scheme $X$ over a field, we have $\operatorname{dim} D^{b}(X-c o h) \leq 2 \operatorname{dim} X$ (Proposition 7.9). We show (Proposition 7.14) that the residue field of a commutative local noetherian algebra $A$ over a field cannot be obtained by less than $\operatorname{Krulldim}(A)$ cones from sums of $A$ and its shifts. This is the key result to get lower bounds : we deduce (Proposition 7.17) that for $X$ a reduced separated scheme of finite type over a field, then $\operatorname{dim} D^{b}(X$-coh $) \geq \operatorname{dim} X$ and there is equality $\operatorname{dim} D^{b}(X$-coh $)=\operatorname{dim} X$ when $X$ is in addition smooth and affine (Theorem 7.18).

In $\S 7.2$, we investigate rings with finite global dimension and regular schemes. As noted by Van den Bergh, a noetherian ring is regular if and only $\operatorname{dim} A$-perf $<\infty$ (Proposition 7.26). Analogously, the category of perfect complexes for a quasi-projective scheme $X$ over a field has finite dimension if and only if $X$ is regular (Proposition 7.35). For an artinian ring $A$, then $\operatorname{dim} D^{b}(A$-mod) is less than the Loewy length (Proposition 7.38).

The main result of $\S 7.4$ is a proof that the derived category of coherent sheaves $D^{b}(X$-coh $)$ has finite dimension, for a separated scheme $X$ of finite type over a perfect field (Theorem 7.39). This is rather surprising and it is a rare instance where $D^{b}(X$-coh) is better behaved than $X$-perf. As a consequence, the stable derived category $D^{b}(X$-coh $) / X$-perf has finite dimension as well. In the smooth case (only), one has a stronger result about the structure sheaf of the diagonal, due to Kontsevich. There are very few cases where we can determine the exact dimension of $D^{b}(X$-coh) for a smooth $X$, and these are cases where it coincides with $\operatorname{dim} X$ ( $X$ affine or $X$ a projective space for example). We conclude the chapter (Corollary 7.50) by a determination of locally finite cohomological functors on $X$-perf and $D^{b}(X \text {-coh })^{\circ}$, for $X$ a projective scheme over a perfect field $k$ (the first case is due to Bondal and Van den Bergh) : they are represented by an object of $D^{b}(X$-coh) in the first case and an object of $X$-perf in the second case - this exhibits some "perfect pairing" $\operatorname{Hom}(-,-): X$-perf $\times D^{b}(X$-coh $) \rightarrow k$-mod.

Finally, in $\$ 8$, we study the dimension of the stable category of a self-injective algebra, in relation with Auslander's representation dimension. Via Koszul duality, we compute these dimensions for the exterior algebra of a finite dimensional vector space : $\operatorname{dim} \Lambda\left(k^{n}\right)$-stab $=n-1$ and the representation dimension of $\Lambda\left(k^{n}\right)$ is $n+1$ (Theorem 8.12). This enables us to settle the characteristic 2 case of a conjecture of Benson (Theorem 8.17).

Preliminary results have been obtained and exposed at the conference "Twenty years of tilting theory" in Fraueninsel in November 2002. I wish to thank the organizers for giving me the opportunity to report on these early results and the participants for many useful discussions, particularly Thorsten Holm for introducing me to Auslander's work.

The geometric part of this work was motivated by lectures given by A. A. Beilinson at the University of Chicago and by discussions with A. Bondal. 


\section{Notations And TERminology}

For $\mathcal{C}$ an additive category and $\mathcal{I}$ a subcategory of $\mathcal{C}$, we denote by $\operatorname{add}(\mathcal{I})(\operatorname{resp} . \overline{\operatorname{add}}(\mathcal{I}))$ the smallest additive full subcategory of $\mathcal{C}$ containing $\mathcal{I}$ and closed under taking direct summands (resp. and closed under direct summands and direct sums). We say that $\mathcal{I}$ is dense if every object of $\mathcal{C}$ is isomorphic to a direct summand of an object of $\mathcal{I}$.

We denote by $\mathcal{C}^{\circ}$ the category opposite to $\mathcal{C}$. We identify a set of objects of $\mathcal{C}$ with the full subcategory with the corresponding set of objects.

Let $\mathcal{T}$ be a triangulated category. A thick subcategory $\mathcal{I}$ of $\mathcal{T}$ is a full triangulated subcategory such that given $M, N \in \mathcal{T}$ with $M \oplus N \in \mathcal{I}$, then $M, N \in \mathcal{I}$. Whenever we consider the quotient $\mathcal{T} / \mathcal{I}$, it will be assumed that this has small Hom-sets.

Given $X \stackrel{f}{\longrightarrow} Y \stackrel{g}{\longrightarrow} Z \rightsquigarrow$ a distinguished triangle, then $Z$ is called a cone of $f$ and $X$ a cocone of $g$.

Given $\mathcal{A}$ an abelian category, we denote by $D(\mathcal{A})$ the derived category of $\mathcal{A}$ and we denote by $D^{\leq a}(\mathcal{A})$ the full subcategory of objects with cohomology vanishing in degrees $>a$.

Let $A$ be a differential graded (=dg) algebra. We denote by $D(A)$ the derived category of dg $A$-modules and by $A$-perf the category of perfect complexes, i.e., the smallest thick subcategory of $D(A)$ containing $A$.

Let $A$ be a ring. We denote by $A$-Mod the category of left $A$-modules, by $A$-mod the category of finitely generated left $A$-modules, by $A$-Proj the category of projective $A$-modules and by $A$-proj the category of finitely generated projective $A$-modules. We denote by gldim $A$ the global dimension of $A$. For $M$ an $A$-module, we denote by $\operatorname{pdim}_{A} M$ the projective dimension of $M$. We denote by $A^{\circ}$ the opposite ring to $A$. For $A$ an algebra over a commutative ring $k$, we put $A^{\text {en }}=A \otimes_{k} A^{\circ}$.

Let $X$ be a scheme. We denote by $X$-coh (resp. $X$-qcoh) the category of coherent (resp. quasi-coherent) sheaves on $X$. We denote by $D(X)$ the full subcategory of the derived category of sheaves of $\mathcal{O}_{X}$-modules consisting of complexes with quasi-coherent cohomology. A complex of sheaves of $\mathcal{O}_{X}$-modules is perfect if it is locally quasi-isomorphic to a bounded complex of vector bundles (=locally free sheaves of finite rank). We denote by $X$-perf the full subcategory of perfect complexes of $D(X)$. Given a complex of sheaves $C$, the notation $H^{i}(C)$ will always refer to the cohomology sheaves, not to the (hyper)cohomology groups.

Let $C$ be a complex of objects of an additive category and $i \in \mathbf{Z}$. We put $\sigma^{\leq i} C=\cdots \rightarrow$ $C^{i-1} \rightarrow C^{i} \rightarrow 0$ and $\sigma^{\geq i} C=0 \rightarrow C^{i} \rightarrow C^{i+1} \rightarrow \cdots$. Let now $C$ be a complex of objects of an abelian category. We put $\tau^{\geq i} C=0 \rightarrow C^{i} / \mathrm{im} d^{i-1} \rightarrow C^{i+1} \rightarrow C^{i+2} \rightarrow \cdots$ and $\tau^{\leq i} C=\cdots \rightarrow$ $C^{i-2} \rightarrow C^{i-1} \rightarrow \operatorname{ker} d^{i} \rightarrow 0$.

\section{Dimension}

\subsection{Dimension for triangulated categories.}

3.1.1. We review here various types of generation of triangulated categories, including the crucial "strong generation" due to Bondal and Van den Bergh.

Let $\mathcal{T}$ be a triangulated category. 
Let $\mathcal{I}_{1}$ and $\mathcal{I}_{2}$ be two subcategories of $\mathcal{T}$. We denote by $\mathcal{I}_{1} * \mathcal{I}_{2}$ the full subcategory of $\mathcal{T}$ consisting of objects $M$ such that there is a distinguished triangle $M_{1} \rightarrow M \rightarrow M_{2} \rightsquigarrow$ with $M_{i} \in \mathcal{I}_{i}$.

Let $\mathcal{I}$ be a subcategory of $\mathcal{T}$. We denote by $\langle\mathcal{I}\rangle$ the smallest full subcategory of $\mathcal{C}$ containing $\mathcal{I}$ and closed under finite direct sums, direct summands and shifts. We denote by $\overline{\mathcal{I}}$ the smallest full subcategory of $\mathcal{C}$ containing $\mathcal{I}$ and closed under direct sums and shifts.

We put $\mathcal{I}_{1} \diamond \mathcal{I}_{2}=\left\langle\mathcal{I}_{1} * \mathcal{I}_{2}\right\rangle$.

We put $\langle\mathcal{I}\rangle_{0}=0$ and we define by induction $\langle\mathcal{I}\rangle_{i}=\langle\mathcal{I}\rangle_{i-1} \diamond\langle\mathcal{I}\rangle$ for $i \geq 1$. We put $\langle\mathcal{I}\rangle_{\infty}=$ $\bigcup_{i>0}\langle\mathcal{I}\rangle_{i}$. We define also $\mathcal{I}^{* i}=\mathcal{I}^{*(i-1)} * \mathcal{I}$.

The objects of $\langle\mathcal{I}\rangle_{i}$ are the direct summands of the objects obtained by taking an $i$-fold extension of finite direct sums of shifts of objects of $\mathcal{I}$.

We will also write $\langle\mathcal{I}\rangle_{\mathcal{T}, i}$ when there is some ambiguity about $\mathcal{T}$.

We say that

- $\mathcal{I}$ generates $\mathcal{T}$ if given $C \in \mathcal{T}$ with $\operatorname{Hom}_{\mathcal{C}}(D[i], C)=0$ for all $D \in \mathcal{I}$ and all $i \in \mathbf{Z}$, then $C=0$

- $\mathcal{I}$ is a d-step generator of $\mathcal{T}$ if $\mathcal{T}=\langle\mathcal{I}\rangle_{d}$ (where $d \in \mathbf{N} \cup\{\infty\}$ )

- $\mathcal{I}$ is a complete d-step generator of $\mathcal{T}$ if $\mathcal{T}=\langle\overline{\mathcal{I}}\rangle_{d}$ (where $d \in \mathbf{N} \cup\{\infty\}$ ).

We say that $\mathcal{T}$ is

- finitely generated if there exists $C \in \mathcal{T}$ which generates $\mathcal{T}$ (such a $C$ is called a generator)

- classically finitely (completely) generated if there exists $C \in \mathcal{T}$ which is a (complete) $\infty$-step generator of $\mathcal{T}$ (such a $C$ is called a classical (complete) generator)

- strongly finitely (completely) generated if there exists $C \in \mathcal{T}$ which is a (complete) $d$-step generator of $\mathcal{T}$ for some $d \in \mathbf{N}$ (such a $C$ is called a strong (complete) generator).

Note that $C$ is a classical generator of $\mathcal{T}$ if and only if $\mathcal{T}$ is the smallest thick subcategory of $\mathcal{T}$ containing $C$. Note also that if $\mathcal{T}$ is strongly finitely generated, then every classical generator is a strong generator.

It will also be useful to allow only certain infinite direct sums. We define $\tilde{\mathcal{I}}$ to be the smallest full subcategory of $\mathcal{T}$ closed under finite direct sums and shifts and containing multiples of objects of $\mathcal{I}$ (i.e., for $X \in \mathcal{I}$ and $E$ a set such that $X^{(E)}$ exists in $\mathcal{T}$, then $X^{(E)} \in \widetilde{\mathcal{I}}$ ).

3.1.2. We now define a dimension for a triangulated category.

Definition 3.1. The dimension of $\mathcal{T}$, denoted by $\operatorname{dim} \mathcal{T}$, is the minimal integer $d \geq 0$ such that there is $M$ in $\mathcal{T}$ with $\mathcal{T}=\langle M\rangle_{d+1}$.

We define the dimension to be $\infty$ when there is no such $M$.

The following Lemmas are clear.

Lemma 3.2. Let $\mathcal{T}^{\prime}$ be a dense full triangulated subcategory of $\mathcal{T}$. Then, $\operatorname{dim} \mathcal{T}=\operatorname{dim} \mathcal{T}^{\prime}$.

Lemma 3.3. Let $F: \mathcal{T} \rightarrow \mathcal{T}^{\prime}$ be a triangulated functor with dense image. If $\mathcal{T}=\langle\mathcal{I}\rangle_{d}$, then $\mathcal{T}^{\prime}=\langle F(\mathcal{I})\rangle_{d}$. So, $\operatorname{dim} \mathcal{T}^{\prime} \leq \operatorname{dim} \mathcal{T}$.

In particular, let $\mathcal{I}$ be a thick subcategory of $\mathcal{T}$. Then, $\operatorname{dim} \mathcal{T} / \mathcal{I} \leq \operatorname{dim} \mathcal{T}$.

Lemma 3.4. Let $\mathcal{T}_{1}$ and $\mathcal{T}_{2}$ be two triangulated subcategories of $\mathcal{T}$ such that $\mathcal{T}=\mathcal{T}_{1} \diamond \mathcal{T}_{2}$. Then, $\operatorname{dim} \mathcal{T} \leq 1+\operatorname{dim} \mathcal{T}_{1}+\operatorname{dim} \mathcal{T}_{2}$ 
Lemma 3.5. The property of generation, strong generation, etc... for $\mathcal{T}$ is equivalent to the corresponding property for $\mathcal{T}^{\circ}$. We have $\operatorname{dim} \mathcal{T}^{\circ}=\operatorname{dim} \mathcal{T}$.

\subsection{Remarks on generation.}

\subsection{1.}

Remark 3.6. One can strengthen the notion of generation of $\mathcal{T}$ by $\mathcal{I}$ by requiring that $\mathcal{T}$ is the smallest triangulated subcategory containing $\mathcal{I}$ and closed under direct sums. Cf Theorem 4.22 for a case where both notions coincide.

Remark 3.7. Let $\langle\mathcal{I}\rangle^{\prime}$ be the smallest full subcategory of $\mathcal{T}$ containing $\mathcal{I}$ and closed under finite direct sums and shifts. Define similarly $\langle\mathcal{I}\rangle_{d}^{\prime}$. Then, $\mathcal{I}$ is a classical generator of $\mathcal{T}$ if and only if the triangulated subcategory $\langle\mathcal{I}\rangle_{\infty}^{\prime}$ of $\mathcal{T}$ is dense. By Thomason's characterization of dense subcategories (Theorem 5.1 below), if $\mathcal{I}$ classically generates $\mathcal{T}$ and the classes of objects of $\mathcal{I}$ generate the abelian group $K_{0}(\mathcal{T})$, then $\mathcal{T}=\langle\mathcal{I}\rangle_{\infty}^{\prime}$.

A similar statement does not hold in general for $d$-step generation, $d \in \mathbf{N}$ : take $\mathcal{T}=$ $D^{b}((k \times k)$-mod $)$, where $k$ is a field. Let $\mathcal{I}$ be the full subcategory containing $k \times k$ and $k \times 0$ (viewed as complexes concentrated in degree 0 ). Then, $\mathcal{T}=\langle\mathcal{I}\rangle$ and $K_{0}(\mathcal{T})=\mathbf{Z} \times \mathbf{Z}$ is generated by the classes of objects of $\mathcal{I}$, but $\langle\mathcal{I}\rangle^{\prime}$ is not a triangulated subcategory of $\mathcal{T}$.

Note the necessity of allowing direct summands when $K_{0}(\mathcal{T})$ is not a finitely generated group (e.g., when $\mathcal{T}=D^{b}(X$-coh) and $X$ is an elliptic curve).

Remark 3.8. It would be interesting to study the "Krull dimension" as well. We say that a thick subcategory $\mathcal{I}$ of $\mathcal{T}$ is irreducible if given two thick subcategories $\mathcal{I}_{1}$ and $\mathcal{I}_{2}$ of $\mathcal{I}$ such that $\mathcal{I}$ is classically generated by $\mathcal{I}_{1} * \mathcal{I}_{2}$, then $\mathcal{I}_{1}=\mathcal{I}$ or $\mathcal{I}_{2}=\mathcal{I}$. We define the Krull dimension of $\mathcal{I}$ as the maximal integer $n$ such that there is a chain of thick irreducible subcategories $0 \neq \mathcal{I}_{0} \subset \mathcal{I}_{1} \subset \cdots \subset \mathcal{I}_{n}=\mathcal{T}$ with $\mathcal{I}_{i} \neq \mathcal{I}_{i+1}$.

By Hopkins-Neeman's Theorem [Nee1, given a commutative noetherian ring $A$, the Krull dimension of the category of perfect complexes of $A$-modules is the Krull dimension of $A$.

By [BeCaRi], given a finite $p$-group $P$, the Krull dimension of the stable category of finite dimensional representations of $P$ over a field of characteristic $p$ is the $p$-rank of $P$ minus 1 .

Another approach would be to study the maximal possible value for the transcendence degree of the field of fractions of the center of $\bigoplus_{i \in \mathbf{Z}} \operatorname{Hom}\left(\mathrm{id}_{\mathcal{T} / \mathcal{I}}, \mathrm{id}_{\mathcal{T} / \mathcal{I}}[i]\right)$, where $\mathcal{I}$ runs over finitely generated thick subcategories of $\mathcal{T}$.

Remark 3.9. When $\mathcal{T}$ has finite dimension, every classical generator is a strong generator. It would be interesting to study the supremum, over all classical generators $M$ of $\mathcal{T}$, of $\min \{d \mid \mathcal{T}=$ $\left.\langle M\rangle_{1+d}\right\}$.

Remark 3.10. One can study also, as a dimension, the minimal integer $d \geq 0$ such that there is $M$ in $\mathcal{T}$ with $\mathcal{T}=\langle\bar{M}\rangle_{d+1}$ or $\mathcal{T}=\langle\widetilde{M}\rangle_{d+1}$ This is of interest for $D(A)$ and $D(X)$ or $D^{b}(A)$ and $D^{b}(X)$.

3.2.2. We often obtain dévissages of objects in the following functorial way (yet another notion of dimension...) :

Assume there are triangulated functors $F_{i}: \mathcal{T} \rightarrow \mathcal{T}$ with image in $\langle\mathcal{I}\rangle$ for $1 \leq i \leq d$, triangulated functors $G_{i}: \mathcal{T} \rightarrow \mathcal{T}$ for $0 \leq i \leq d$ with $G_{0}=\mathrm{id}, G_{d}=0$ and distinguished triangles $F_{i} \rightarrow G_{i} \rightarrow G_{i-1} \rightsquigarrow$ for $1 \leq i \leq d$. Then, $\mathcal{T}=\langle\mathcal{I}\rangle_{d}$. 


\subsection{Compact objects.}

3.3.1. Let $\mathcal{C}$ be an additive category. We say that $\mathcal{C}$ is cocomplete if arbitrary direct sums exist in $\mathcal{C}$.

An object $C \in \mathcal{C}$ is compact if for every set $\mathcal{F}$ of objects of $\mathcal{C}$ such that $\bigoplus_{F \in \mathcal{F}} F$ exists, then the canonical map $\bigoplus_{F \in \mathcal{F}} \operatorname{Hom}(C, F) \rightarrow \operatorname{Hom}\left(C, \bigoplus_{F \in \mathcal{F}} F\right)$ is an isomorphism. We denote by $\mathcal{C}^{c}$ the set of compact objects of $\mathcal{C}$.

A triangulated category $\mathcal{T}$ is compactly generated if it generated by a set of compact objects. We say that a full triangulated subcategory $\mathcal{I}$ of $\mathcal{T}$ is compactly generated in $\mathcal{T}$ if it is generated by a set of objects of $\mathcal{I} \cap \mathcal{T}^{c}$.

3.3.2. Let $\mathcal{T}$ be a triangulated category. Then, $\mathcal{T}^{c}$ is a thick subcategory of $\mathcal{T}$.

Let $X_{0} \stackrel{s_{0}}{\longrightarrow} X_{1} \stackrel{s_{1}}{\longrightarrow} \cdots$ be a sequence of objects and maps of $\mathcal{T}$. If $\bigoplus_{i \geq 0} X_{i}$ exists, then the homotopy colimit of the sequence, denoted by hocolim $X_{i}$, is a cone of the morphism $\sum_{i} \operatorname{id}_{X_{i}}-s_{i}: \bigoplus_{i \geq 0} X_{i} \rightarrow \bigoplus_{i \geq 0} X_{i}$

We have a canonical map

$$
\operatorname{colim}_{\operatorname{Hom}}\left(Y, X_{i}\right) \rightarrow \operatorname{Hom}_{\mathcal{T}}\left(Y, \operatorname{hocolim} X_{i}\right)
$$

that makes the following diagram commutative

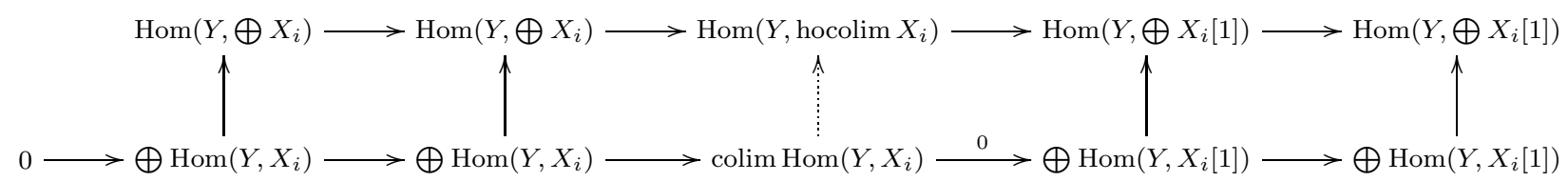

Since the horizontal sequences of the diagram above are exact, we deduce (cf e.g. Nee2, Lemma 1.5]) :

Lemma 3.11. The canonical map colim $\operatorname{Hom}_{\mathcal{T}}\left(Y, X_{i}\right) \rightarrow \operatorname{Hom}_{\mathcal{T}}\left(Y\right.$, hocolim $\left.X_{i}\right)$ is an isomorphism if $Y$ is compact.

We now combine the commutation of $\operatorname{Hom}(Y,-)$ with colimits and with direct sums in the following result (making more precise a classical result [Nee2, Lemma 2.3]) :

Proposition 3.12. Let $0=X_{0} \rightarrow X_{1} \rightarrow X_{2} \rightarrow \cdots$ be a directed system in $\mathcal{T}$, let $\mathcal{F}_{i}$ be a set of compact objects such that $\bigoplus_{C \in \mathcal{F}_{i}} C$ exists and let $X_{i-1} \rightarrow X_{i} \rightarrow \bigoplus_{C \in \mathcal{F}_{i}} C \rightsquigarrow$ be a distinguished triangle, for $i \geq 1$.

Let $Y$ be a compact object and $f: Y \rightarrow$ hocolim $X_{i}$. Then, there is an integer $d \geq 1$, a finite subset $\mathcal{F}_{i}^{\prime}$ of $\mathcal{F}_{i}$ for $1 \leq i \leq d$ and a commutative diagram

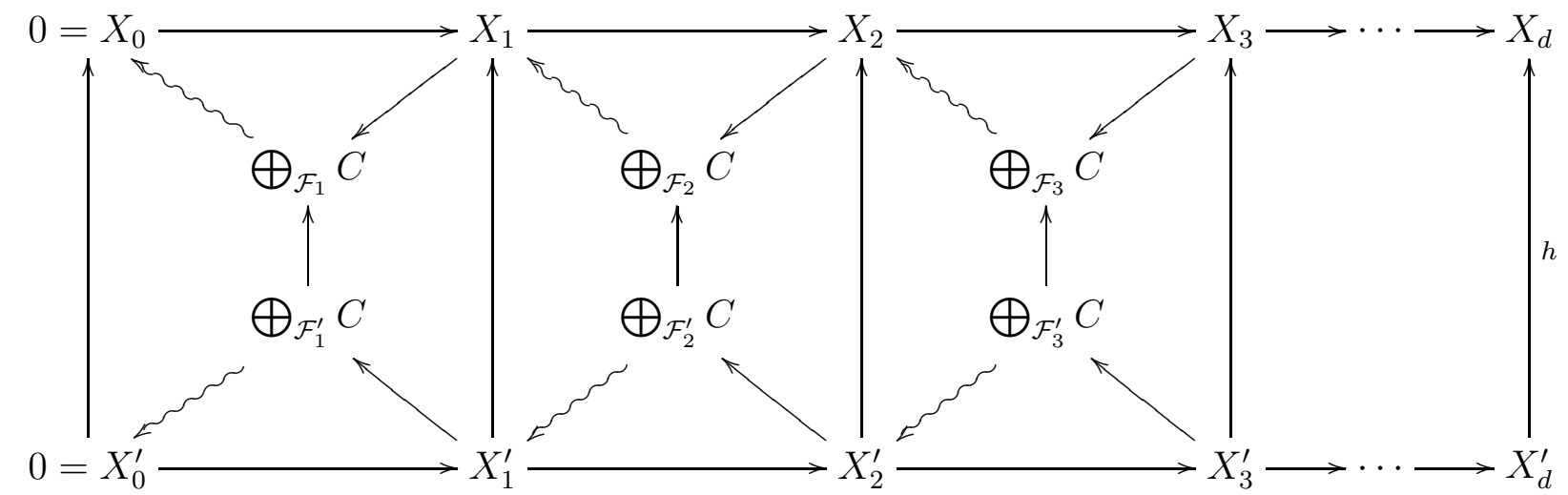


such that $f$ factors through $X_{d}^{\prime} \stackrel{h}{\longrightarrow} X_{d} \stackrel{\text { can }}{\longrightarrow}$ hocolim $X_{i}$.

Proof. By Lemma 3.11, there is $d \geq 1$ such that $f$ factors through the canonical map $X_{d} \rightarrow$ hocolim $X_{i}$. We proceed now by induction on $d$. The composite map $Y \rightarrow X_{d} \rightarrow \bigoplus_{C \in \mathcal{F}_{d}} C$ factors through the sum indexed by a finite subset $\mathcal{F}_{d}^{\prime}$ of $\mathcal{F}_{d}$. Let $Z$ be the cocone of the corresponding map $Y \rightarrow \bigoplus_{C \in \mathcal{F}_{d}^{\prime}} C$ and $X_{d}^{\prime \prime}$ the cocone of the composite map $\bigoplus_{C \in \mathcal{F}_{d}^{\prime}} C \rightarrow$ $\bigoplus_{C \in \mathcal{F}_{d}} C \rightarrow X_{d-1}[1]$. The composite map $X_{d}^{\prime \prime} \rightarrow \bigoplus_{C \in \mathcal{F}_{d}^{\prime}} C \rightarrow \bigoplus_{C \in \mathcal{F}_{d}} C$ factors through $X_{d}$. The map $Y \rightarrow X_{d}$ factors through $X_{d}^{\prime \prime}$ and the composite map $Z \rightarrow Y \rightarrow X_{d}^{\prime \prime}$ factors through $X_{d-1}$. Summarizing, we have a commutative diagram

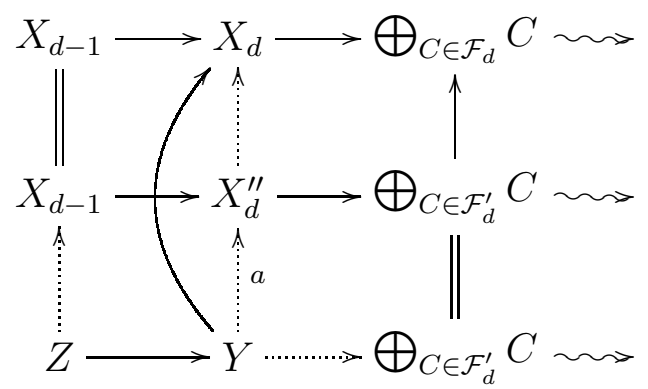

By induction, we have already a commutative diagram as in the proposition for the corresponding map $Z \rightarrow X_{d-1}$. We define now $X_{d}^{\prime}$ to be the cocone of the composite map $\bigoplus_{C \in \mathcal{F}_{d}^{\prime}} C \rightarrow Z[1] \rightarrow X_{d-1}^{\prime}[1]$. There is a commutative diagram

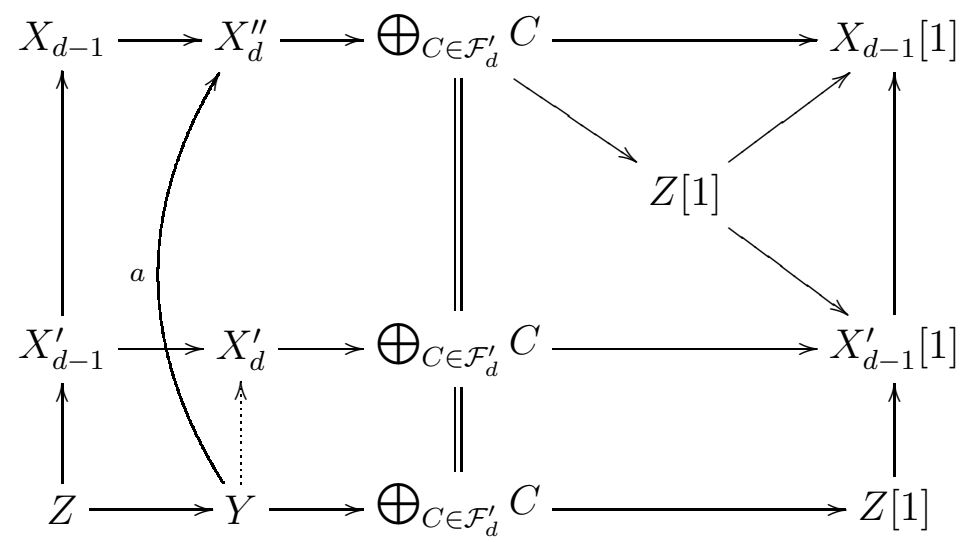

The composite map $Z \rightarrow Y \rightarrow X_{d}^{\prime \prime}$ factors through $X_{d-1}$, hence through $X_{d-1}^{\prime}$. It follows that $a$ factors through $X_{d}^{\prime}$ and we are done.

We deduce the following descent result $[\mathrm{BoVdB}$, Proposition 2.2.4] :

Corollary 3.13. Let $\mathcal{I}$ be a subcategory of $\mathcal{T}^{c}$ and let $d \in \mathbf{N} \cup\{\infty\}$. Then, $\mathcal{T}^{c} \cap\langle\overline{\mathcal{I}}\rangle_{d}=\langle\mathcal{I}\rangle_{d}$.

Proof. Let $Y$ be a compact object and $f: Y \rightarrow X_{d}$ be a split injection where $X_{d}$ is obtained by taking a $d$-fold extension of objects of $\langle\overline{\mathcal{I}}\rangle$. Proposition 3.12 shows that $f$ factors through an object $X_{d}^{\prime} \in\langle\mathcal{I}\rangle_{d}$ and we obtain a split injection $Y \rightarrow X_{d}^{\prime}$.

3.4. Relation with dg algebras. Following Keller, we say that a triangulated category $\mathcal{T}$ is algebraic if it is the stable category of a Frobenius exact category [GeMa, Chapter 5, §2.6] (for example, $\mathcal{T}$ can be the derived category of an abelian category). 
Recall the construction of $[\mathrm{Ke}, \S 4.3]$. Let $\mathcal{T}=\mathcal{E}$-stab be the stable category of a Frobenius exact category $\mathcal{E}$. Let $\mathcal{E}^{\prime}$ be the category of acyclic complexes of projective objects of $\mathcal{E}$ and $Z^{0}: \mathcal{E}^{\prime} \rightarrow \mathcal{E}$-stab be the functor that sends $C$ to coker $d_{C}^{-1}$.

Given $X$ and $Y$ two complexes of objects of $\mathcal{E}$, we denote by $\operatorname{Hom}^{\bullet}(X, Y)$ the total Hom complex (i.e., $\operatorname{Hom}^{\bullet}(X, Y)^{i}=\prod_{j \in \mathbf{Z}} \operatorname{Hom}_{\mathcal{E}}\left(X^{j}, Y^{i+j}\right)$ ).

Let $M \in \mathcal{E}$-stab and $M^{\prime} \in \mathcal{E}^{\prime}$ with $Z^{0}\left(M^{\prime}\right) \stackrel{\sim}{\rightarrow} M$. Let $A=\operatorname{End}^{\bullet}\left(M^{\prime}\right)$ be the dg algebra of endomorphisms of $M^{\prime}$. The functor $\operatorname{Hom} \bullet\left(M^{\prime},-\right): \mathcal{E}^{\prime} \rightarrow D(A)$ factors through $Z^{0}$ and induces a triangulated functor $R \operatorname{Hom}^{\bullet}(M,-): \mathcal{E}$-stab $\rightarrow D(A)$. That functor restricts to an equivalence $\langle M\rangle_{\infty} \stackrel{\sim}{\rightarrow} A$-perf. In particular, if $M$ is a classical generator of $\mathcal{T}$, then we get the equivalence $\mathcal{T} \stackrel{\sim}{\rightarrow} A$-perf.

So,

Proposition 3.14. Let $\mathcal{T}$ be an algebraic triangulated category. Then, $\mathcal{T}$ is classically finitely generated if and only if it is equivalent to the category of perfect complexes over a dg algebra.

This should be compared with the following result.

Assume now $\mathcal{E}$ is a cocomplete Frobenius category (i.e., all direct sums exist and are exact). If $M$ is compact, then $R \operatorname{Hom}^{\bullet}(M,-)$ restricts to an equivalence between the smallest full triangulated subcategory of $\mathcal{T}$ containing $M$ and closed under direct sums and $D(A)$ (cf Theorem 4.22 (2) and Corollary 6.1 below). So, using Theorem 4.22 (2) below, we deduce $\mathbb{K}$, Theorem 4.3] :

Theorem 3.15. Let $\mathcal{E}$ be a cocomplete Frobenius category and $\mathcal{T}=\mathcal{E}$-stab. Then, $\mathcal{T}$ has a compact generator if and only if it is equivalent to the derived category of a dg algebra.

\section{Finiteness CONDitions AND REPRESEntabiLity}

4.1. Finiteness for cohomological functors. We introduce a class of "locally finitely presented" cohomological functors that includes the representable functors, inspired by Brown's representability Theorem. It extends the class of locally finite functors, of interest only for Ext-finite triangulated categories.

\subsubsection{Let $k$ be a commutative ring.}

Let $\mathcal{T}$ be a $k$-linear triangulated category. Let $H: \mathcal{T}^{\circ} \rightarrow k$-Mod be a ( $k$-linear) functor. We say that $H$ is cohomological if for every distinguished triangle $X \stackrel{f}{\longrightarrow} Y \stackrel{g}{\longrightarrow} Z \rightsquigarrow$, then the associated sequence $H(Z) \stackrel{H(g)}{\longrightarrow} H(Y) \stackrel{H(f)}{\longrightarrow} H(X)$ is exact.

For $C \in \mathcal{T}$, we denote by $h_{C}$ the cohomological functor $\operatorname{Hom}_{\mathcal{T}}(-, C): \mathcal{T}^{\circ} \rightarrow k$-Mod.

We will repeatedly use Yoneda's Lemma :

Lemma 4.1. Let $X \in \mathcal{T}$ and $H: \mathcal{T}^{\circ} \rightarrow k$-Mod a functor. Then, the canonical map $\operatorname{Hom}\left(h_{C}, H\right) \rightarrow H(C), f \mapsto f(C)\left(\operatorname{id}_{C}\right)$ is an isomorphism.

Let $H: \mathcal{T}^{\circ} \rightarrow k$-Mod be a functor. We say that $H$ is

- locally bounded (resp. bounded above, resp. bounded below) if for every $X \in \mathcal{T}$, we have $H(X[i])=0$ for $|i| \gg 0$ (resp. for $i \ll 0$, resp. for $i \gg 0$ )

- locally finitely generated if for every $X \in \mathcal{T}$, there is $D \in \mathcal{T}$ and $\alpha: h_{D} \rightarrow H$ such that $\alpha(X[i])$ is surjective for all $i$. 
- locally finitely presented if it is locally finitely generated and the kernel of any map $h_{E} \rightarrow H$ is locally finitely generated.

Let $X \in \mathcal{T}$. We introduce two conditions :

(a) there is $D \in \mathcal{T}$ and $\alpha: h_{D} \rightarrow H$ such that $\alpha(X[i])$ is surjective for all $i$

(b) for every $\beta: h_{E} \rightarrow H$, there is $f: F \rightarrow E$ such that $\beta h_{f}=0$ and $h_{F}(X[i]) \stackrel{h_{f}}{\longrightarrow}$ $h_{E}(X[i]) \stackrel{\beta}{\longrightarrow} H(X[i])$ is an exact sequence for all $i$.

Note that $H$ is locally finitely presented if and only if for every $X \in \mathcal{T}$, then conditions (a) and (b) are fulfilled.

Lemma 4.2. For $C \in \mathcal{T}$, then $h_{C}$ is locally finitely presented.

Proof. We take $D=C$ and $\alpha=$ id for condition (a). For (b), a map $\beta: h_{E} \rightarrow h_{C}$ comes from a map $g: E \rightarrow C$ and we pick a distinguished triangle $F \stackrel{f}{\longrightarrow} E \stackrel{g}{\longrightarrow} C \rightsquigarrow$.

Proposition 4.3. Let $H_{0} \rightarrow H_{1} \rightarrow H \rightarrow H_{2} \rightarrow H_{3}$ be an exact sequence of functors $\mathcal{T}^{\circ} \rightarrow$ $k$-Mod.

If $H_{1}$ and $H_{2}$ are locally finitely generated and $H_{3}$ is locally finitely presented, then $H$ is locally finitely generated.

If $H_{0}$ is locally finitely generated and $H_{1}, H_{2}$ and $H_{3}$ are locally finitely presented, then $H$ is locally finitely presented.

Proof. Let us name the maps : $H_{0} \stackrel{t_{0}}{\longrightarrow} H_{1} \stackrel{t_{1}}{\longrightarrow} H \stackrel{t_{2}}{\longrightarrow} H_{2} \stackrel{t_{3}}{\longrightarrow} H_{3}$. Let $X \in \mathcal{T}$.

Let $\alpha_{2}: h_{D_{2}} \rightarrow H_{2}$ as in (a). Let $\beta_{3}=t_{3} \alpha_{2}: h_{D_{2}} \rightarrow H_{3}$. Let $f_{3}: E \rightarrow D_{2}$ as in (b). Since $H(E) \rightarrow H_{2}(E) \rightarrow H_{3}(E)$ is exact, the composite map $\alpha_{2} h_{f_{3}}: h_{E} \rightarrow H_{2}$ factors as $h_{E} \stackrel{\gamma}{\longrightarrow} H \stackrel{t_{2}}{\longrightarrow} H_{2}$. Let $\alpha_{1}: h_{D_{1}} \rightarrow H_{1}$ as in (a).

Let $a: h_{X} \rightarrow H$. The composite $t_{2} a: h_{X} \rightarrow H_{2}$ factors as $t_{2} a: h_{X} \stackrel{b}{\longrightarrow} h_{D_{2}} \stackrel{\alpha_{2}}{\longrightarrow} H_{2}$. The composition $t_{3}\left(t_{2} a\right): h_{X} \rightarrow H_{3}$ is zero, hence $b$ factors as $b: h_{X} \stackrel{c}{\longrightarrow} h_{E} \stackrel{h_{f_{3}}}{\longrightarrow} h_{D_{2}}$. Now, we have $t_{2} \gamma c=\alpha_{2} h_{f_{3}} c=\alpha_{2} b=t_{2} a$. Since the composite $t_{2}(a-\gamma c): h_{X} \rightarrow H_{2}$ is zero, it follows that $a-\gamma c$ factors as $h_{X} \stackrel{a_{1}}{\longrightarrow} H_{1} \stackrel{t_{1}}{\longrightarrow} H$. Now, $a_{1}$ factors through $\alpha_{1}$. So, we have shown that $a$ factors through $\gamma+t_{1} \alpha_{1}: h_{E} \oplus h_{D_{1}} \rightarrow H$, hence $H$ satisfies (a).

Let $\alpha_{0}: h_{D_{0}} \rightarrow H_{0}$ as in (a). Let $\beta^{\prime}: h_{E} \rightarrow \operatorname{ker} t_{2}$. Then, there is $\beta_{1}: h_{E} \rightarrow H_{1}$ such that $\beta^{\prime}=t_{1} \beta_{1}$. Since $H_{1}$ is locally finitely presented, there are $u: h_{F} \rightarrow h_{D_{0}}$ and $v: h_{F} \rightarrow h_{E}$ such that $\left(\beta_{1}+t_{0} \alpha_{0}\right)(u-v)=0$ and $h_{F}(X[i]) \stackrel{u-v}{\longrightarrow} h_{E}(X[i]) \oplus h_{D_{0}}(X[i]) \stackrel{\beta_{1}+t_{0} \alpha_{0}}{\longrightarrow} H_{1}(X[i])$ is exact for every $i$. Summarizing, we have a commutative diagram

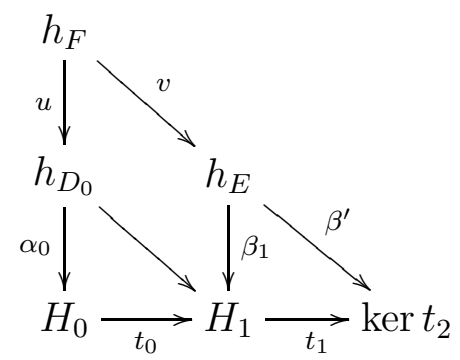

It follows that $\beta v=0$ and $h_{F}(X[i]) \stackrel{v}{\longrightarrow} h_{E}(X[i]) \stackrel{\beta}{\longrightarrow}\left(\operatorname{ker} t_{2}\right)(X[i])$ is exact for every $i$, hence ker $t_{2}$ satisfies (b). 
Let now $\beta: h_{E} \rightarrow H$. Let $G=\operatorname{ker} \beta$ and $G_{2}=\operatorname{ker}\left(t_{2} \beta\right)$. Now, we have exact sequences $0 \rightarrow G \rightarrow G_{2} \rightarrow$ ker $t_{2}$ and $0 \rightarrow G_{2} \rightarrow h_{E} \rightarrow H_{2}$. The first part of the Proposition together with Lemma 4.2 shows that $G$ is finitely generated. Consequently, $H$ is locally finitely generated.

4.1.2. We will now study conditions (a) and (b) in the definition of locally finitely presented functors.

Lemma 4.4. Let $H: \mathcal{T}^{\circ} \rightarrow k$-Mod be a $k$-linear functor and $X \in \mathcal{T}$.

- Let $\beta_{r}: h_{E_{r}} \rightarrow H$ for $r \in\{1,2\}$ such that (b) holds for $\beta=\beta_{1}+\beta_{2}: h_{E_{1} \oplus E_{2}} \rightarrow H$. Then, (b) holds for $\beta_{1}$ and $\beta_{2}$.

- Assume (a) holds. If (b) holds for those $\beta: h_{E} \rightarrow H$ such that $\beta(X[i])$ is surjective for all $i$, then (b) holds for all $\beta$.

Proof. Let $E=E_{1} \oplus E_{2}$. Denote by $i_{r}: E_{r} \rightarrow E$ and $p_{r}: E \rightarrow E_{r}$ the injections and projections.

There is $f: F \rightarrow E$ such that $\beta h_{f}=0$ and $h_{F}(X[i]) \stackrel{h_{f}}{\longrightarrow} h_{E}(X[i]) \stackrel{\beta}{\longrightarrow} H(X[i])$ is an exact sequence for all $i$.

Fix a distinguished triangle $F_{1} \stackrel{f_{1}^{\prime}}{\longrightarrow} F \stackrel{p_{2} f}{\longrightarrow} E_{2} \rightsquigarrow$ and let $f_{1}=p_{1} f f_{1}^{\prime}: F_{1} \rightarrow E_{1}$. We have $\beta_{1} h_{f_{1}}=0$ since $\beta_{1} h_{p_{1} f}=-\beta_{2} h_{p_{2} f}$.

For all $i$, the horizontal sequences and the middle vertical sequence in the following commutative diagram are exact

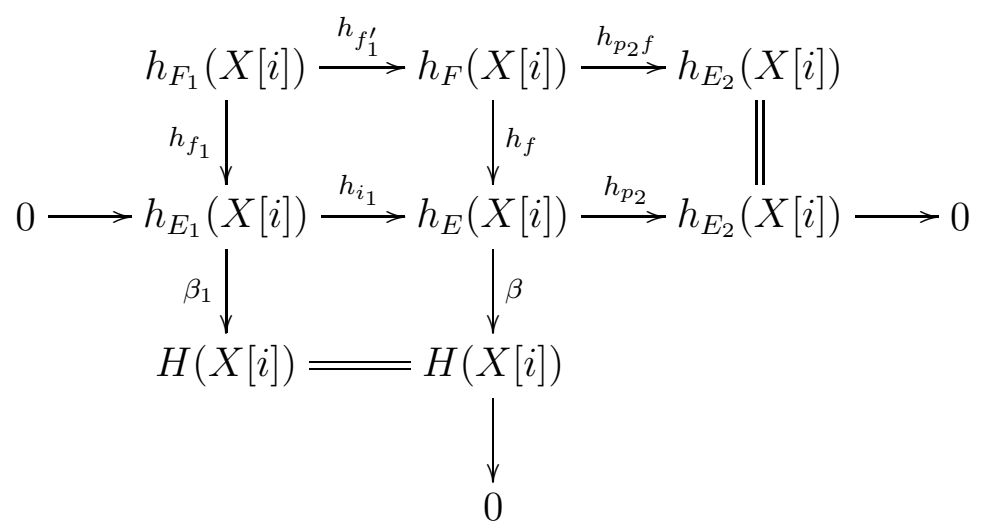

hence the left vertical sequence is exact as well.

Let us now prove the second part of the Lemma. Let $\beta: h_{E} \rightarrow H$. Since (a) holds, there is $D \in \mathcal{T}$ and $\alpha: h_{D} \rightarrow H$ such that $\alpha(X[i])$ is surjective for all $i$. Let $E^{\prime}=D \oplus E$ and $\beta^{\prime}=\alpha+\beta: h_{E^{\prime}} \rightarrow H$. Then, (b) holds for $\beta^{\prime}$, hence it holds for $\beta$ by the first part of the Lemma.

Remark 4.5. For the representability Theorem (cf Lemma 4.14), only the surjective case of (b) is needed, but the previous Lemma shows that this implies that (b) holds in general.

Lemma 4.6. Let $H: \mathcal{T}^{\circ} \rightarrow k$-Mod be a cohomological functor.

The full subcategory of $X$ in $\mathcal{T}$ such that (a) and (b) hold is a thick triangulated subcategory of $\mathcal{T}$.

In particular, if $X$ is a classical generator for $\mathcal{T}$ and (a), (b) hold, then $H$ is locally finitely presented. 
Proof. Let $\mathcal{I}$ be the full subcategory of those $X$ such that (a) and (b) hold. It is clear that $\mathcal{I}$ is closed under shifts and under taking direct summands. So, we are left with proving that $\mathcal{I}$ is stable under extensions.

Let $X_{1} \stackrel{u}{\longrightarrow} X \rightarrow X_{2} \rightsquigarrow$ be a distinguished triangle in $\mathcal{T}$ with $X_{1}, X_{2} \in \mathcal{I}$. Pick $D_{r} \in$ $\mathcal{T}$ and $\alpha_{r}: h_{D_{r}} \rightarrow H$ such that $\alpha_{r}\left(X_{r}[i]\right)$ is surjective for all $i$. Put $E=D_{1} \oplus D_{2}$ and $\beta=\alpha_{1}+\alpha_{2}: h_{E} \rightarrow H$. There is $F_{r} \in \mathcal{T}$ and $f_{r}: F_{r} \rightarrow E$ such that $\beta h_{f_{r}}=0$ and $h_{F_{r}}\left(X_{r}[i]\right) \stackrel{h_{f}}{\longrightarrow} h_{E}\left(X_{r}[i]\right) \stackrel{\beta}{\longrightarrow} H\left(X_{r}[i]\right)$ is an exact sequence for all $i$. Put $F=F_{1} \oplus F_{2}$ and $f=f_{1}+f_{2}: F \rightarrow E$. Let $F \stackrel{f}{\longrightarrow} E \stackrel{t}{\longrightarrow} E^{\prime} \rightsquigarrow$ be a distinguished triangle. We have an exact sequence $H\left(E^{\prime}\right) \rightarrow H(E) \rightarrow H(F)$. The image in $H(F)$ of the element of $H(E)$ corresponding to $\beta$ is 0 , since $\beta h_{f}=0$. Hence, $\beta$ factors as $h_{E} \stackrel{h_{t}}{\longrightarrow} h_{E^{\prime}} \stackrel{\gamma}{\longrightarrow} H$. Let $D=E \oplus E^{\prime}$ and $\alpha=\beta+\gamma: h_{D} \rightarrow H$.

Let $a: h_{X} \rightarrow H$. Then, there is a commutative diagram where the top horizontal sequence is exact

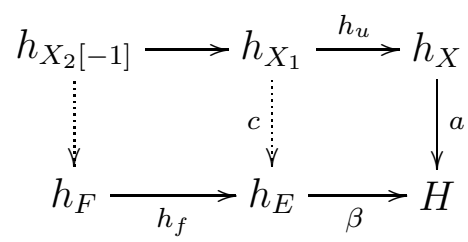

The composite $h_{X_{2}[-1]} \rightarrow h_{X_{1}} \stackrel{c}{\longrightarrow} h_{E} \stackrel{h_{t}}{\longrightarrow} h_{E^{\prime}}$ is zero, hence $h_{t} c: h_{X_{1}} \rightarrow h_{E^{\prime}}$ factors as $h_{X_{1}} \stackrel{h_{u}}{\longrightarrow} h_{X} \stackrel{b}{\longrightarrow} h_{E^{\prime}}$. We have $a h_{u}=\beta c=\gamma h_{t} c=\gamma b h_{u}$, hence the composite $h_{X_{1}} \rightarrow h_{X} \stackrel{a^{\prime}}{\longrightarrow} H$ is zero, where $a^{\prime}=a-\gamma b$. So, $a^{\prime}$ factors through a map $h_{X_{2}} \rightarrow H$. Such a map factors through $\beta$, hence $a^{\prime}$ factors through $\beta$ and $a$ factors through $\alpha$. The same conclusion holds for $a$ replaced by any map $h_{X[i]} \rightarrow H$ for some $i \in \mathbf{Z}$. So, every map $h_{X[i]} \rightarrow H$ factors through $\alpha$, i.e., (a) holds for $X$.

Consider now a map $\beta^{\prime}: h_{E^{\prime}} \rightarrow H$. Let $\beta^{\prime \prime}: h_{E^{\prime \prime}} \rightarrow H$ such that $\beta^{\prime \prime}\left(X_{1}[i]\right)$ is surjective for all $i$. Let $\beta=\beta^{\prime}+\beta^{\prime \prime}: h_{E} \rightarrow H$, where $E=E^{\prime} \oplus E^{\prime \prime}$. In order to prove that $\beta^{\prime}$ satisfies (b), it suffices to prove that $\beta$ satisfies (b), thanks to Lemma 4.4.

There is $F_{1} \in \mathcal{T}$ and $f_{1}: F_{1} \rightarrow E$ such that $\beta h_{f_{1}}=0$ and $h_{F_{1}}\left(X_{1}[i]\right) \stackrel{h_{f_{1}}}{\longrightarrow} h_{E}\left(X_{1}[i]\right) \stackrel{\beta}{\longrightarrow}$ $H\left(X_{1}[i]\right)$ is an exact sequence for all $i$. Let $E_{1}$ be the cone of $f_{1}$. As in the discussion above, $\beta$ factors through a map $\gamma: h_{E_{1}} \rightarrow H$. Let $F_{2} \in \mathcal{T}$ and $f_{2}: F_{2} \rightarrow E_{1}$ such that $\gamma h_{f_{2}}=0$ and $h_{F_{2}}\left(X_{2}[i]\right) \stackrel{h_{f_{2}}}{\longrightarrow} h_{E_{1}}\left(X_{2}[i]\right) \stackrel{\gamma}{\longrightarrow} H\left(X_{2}[i]\right)$ is an exact sequence for all $i$. Let $F$ be the cocone of the sum map $E \oplus F_{2} \rightarrow E_{1}$. The composition $h_{F} \rightarrow h_{E} \stackrel{\beta}{\longrightarrow} H$ is zero. We have a commutative diagram

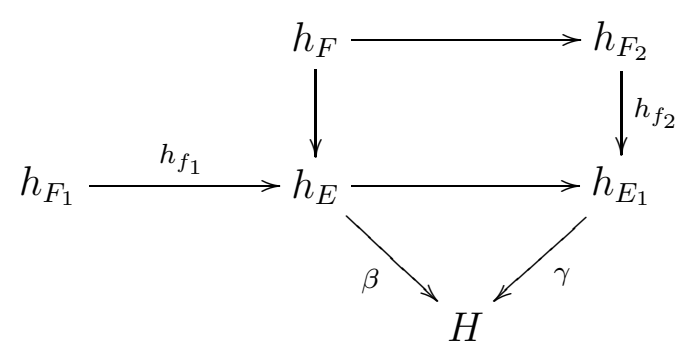


In the diagram, the square is homotopy cartesian, i.e., given $Y \in \mathcal{T}$ and $u: Y \rightarrow E, v: Y \rightarrow F_{2}$ such that the compositions $Y \stackrel{u}{\longrightarrow} E \rightarrow E_{1}$ and $Y \stackrel{v}{\longrightarrow} F_{2} \rightarrow E_{1}$ are equal, then there is $w: Y \rightarrow F$ such that $u$ is the composition $Y \stackrel{w}{\longrightarrow} F \rightarrow E$ and $v$ the composition $Y \stackrel{w}{\longrightarrow} F \rightarrow F_{2}$.

Let $a: h_{X} \rightarrow h_{E}$ such that $\beta a=0$. The composite $h_{X_{1}} \rightarrow h_{X} \stackrel{a}{\longrightarrow} h_{E}$ factors through $h_{F_{1}}$. It follows that the composition $h_{X_{1}} \rightarrow h_{X} \stackrel{a}{\longrightarrow} h_{E} \rightarrow h_{E_{1}}$ is zero. Hence, the composite $h_{X} \stackrel{a}{\longrightarrow} h_{E} \rightarrow h_{E_{1}}$ factors through a map $b: h_{X_{2}} \rightarrow h_{E_{1}}$. The composite $b^{\prime}: h_{X_{2}} \stackrel{b}{\longrightarrow} h_{E_{1}} \rightarrow H$ factors through a map $c: h_{X_{1}[1]} \rightarrow H$, since $h_{X} \rightarrow h_{X_{2}} \stackrel{b^{\prime}}{\longrightarrow} H$ is zero. Now, $c$ factors as $h_{X_{1}[1]} \stackrel{d}{\longrightarrow} h_{E_{1}} \stackrel{\gamma}{\longrightarrow} H$. Summarizing, we have a diagram all of whose squares and triangles but the one marked " $\neq$ " are commutative and where the horizontal sequences are exact

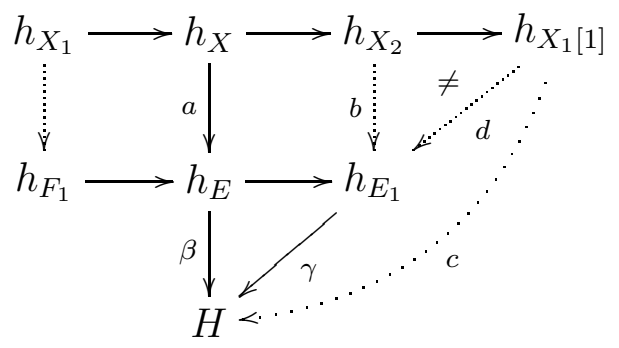

Let $d^{\prime}$ be the composition $h_{X_{2}} \rightarrow h_{X_{1}[1]} \stackrel{d}{\longrightarrow} h_{E_{1}}$. Then, the composition $h_{X_{2}} \stackrel{b-d^{\prime}}{\longrightarrow} h_{E_{1}} \stackrel{\gamma}{\longrightarrow} H$ is zero. The map $b-d^{\prime}$ factors as $h_{X_{2}} \stackrel{d^{\prime \prime}}{\longrightarrow} h_{F_{2}} \stackrel{h_{f_{2}}}{\longrightarrow} h_{E_{1}}$. It follows that $h_{X} \stackrel{a}{\longrightarrow} h_{E} \rightarrow h_{E_{1}}$ factors as $h_{X} \rightarrow h_{X_{2}} \stackrel{d^{\prime \prime}}{\longrightarrow} h_{F_{2}} \stackrel{h_{f_{2}}}{\longrightarrow} h_{E_{1}}$. Using the homotopy cartesian square above, we deduce that $a$ factors through $a^{\prime}: h_{X} \rightarrow h_{F}$. So, the sequence $h_{F}(X) \rightarrow h_{E}(X) \rightarrow H(X)$ is exact. The same holds for all $i$, hence (b) holds for $X$.

Remark 4.7. All the results concerning locally finitely generated and presented functors above remain valid if we replace the conditions "given $X \in \mathcal{T}$, a certain statement is true for all $i \in \mathbf{Z}$ " by "given $X \in \mathcal{T}$ and $a \in \mathbf{Z}$, a certain statement is true for $i \geq a$ " (or " $i \leq a$ " or " $i=a$ ") in (a) and (b). Cf for example Proposition 6.10.

4.1.3.

Proposition 4.8. Let $H: \mathcal{T}^{\circ} \rightarrow k$-Mod be a cohomological functor and $X$ be a classical generator for $\mathcal{T}$. Then, $H$ is locally finitely generated if and only if $\bigoplus_{i} H(X[i])$ is a finitely generated $\operatorname{End}^{*}(X)$-module.

Proof. Assume first $\bigoplus_{i} H(X[i])$ is a finitely generated $\operatorname{End}^{*}(X)$-module. Let $f_{r} \in \operatorname{Hom}\left(h_{X\left[n_{r}\right]}, H\right)$ be a finite set of elements such that the $f_{r}\left(X\left[n_{r}\right]\right)\left(\operatorname{id}_{X\left[n_{r}\right]}\right)$ generate $\bigoplus_{i} H(X[i])$ as an $\operatorname{End}^{*}(X)$ module. Let $D=\bigoplus_{r} X\left[n_{r}\right]$ and $f=\sum_{r} f_{r}: h_{D} \rightarrow H$. Then, $f(X[i])$ is surjective for every $i$, i.e., condition (a) is satisfied.

Conversely, assume (a) is satisfied.

4.1.4. Assume $k$ is noetherian. We say that $\mathcal{T}$ is Ext-finite if $\bigoplus_{i} \operatorname{Hom}(X, Y[i])$ is a finitely generated $k$-module, for every $X, Y \in \mathcal{T}$.

Assume now $\mathcal{T}$ is Ext-finite and let $H: \mathcal{T}^{\circ} \rightarrow k$-Mod be a functor. We say that $H$ is locally finite if for every $X \in \mathcal{T}$, the $k$-module $\bigoplus_{i} H(X[i])$ is finitely generated.

Proposition 4.9. Let $H$ be a locally finite functor. Then, $H$ is locally bounded and locally finitely presented. 
Proof. It is clear that $H$ is locally bounded. Let $X \in \mathcal{T}$. Let $I_{i}$ be a minimal (finite) family of generators of $H(X[i])$ as a $k$-module. We have $I_{i}=\emptyset$ for almost all $i$, since $H$ is locally bounded. Put $D=\bigoplus_{i} X[i] \otimes_{k} k^{I_{i}}$ and let $\alpha: h_{D} \rightarrow H$ be the canonical map. The map $\alpha(X[i])$ is surjective for all $i$. So, every locally finite functor is locally finitely generated.

Let now $\beta: h_{E} \rightarrow H$. Let $G=\operatorname{ker} \beta$. Since $\mathcal{T}$ is Ext-finite, $G$ is again locally finite, hence locally finitely generated.

The results on finitely generated and presented functors discussed above have counterparts for locally bounded functors, the proofs being trivial in this case.

Proposition 4.10. Let $H_{1} \rightarrow H \rightarrow H_{2}$ be an exact sequence of functors $\mathcal{T}^{\circ} \rightarrow k$-Mod. If $H_{1}$ and $H_{2}$ are locally bounded (resp. bounded above, resp. bounded below), then $H$ is locally bounded (resp. bounded above, resp. bounded below).

Let $H: \mathcal{T}^{\circ} \rightarrow k$-Mod be a cohomological functor. Then, the full subcategory of $X \in \mathcal{T}$ such that $H(X[i])=0$ for $|i| \gg 0$ (resp. $i \ll 0$, resp. $i \gg 0)$ is a thick subcategory.

\subsection{Locally finitely presented functors.}

4.2.1. Let us start with some remarks on cohomological functors.

Given $0 \rightarrow H_{1} \rightarrow H_{2} \rightarrow H_{3} \rightarrow 0$ an exact sequence of functors $\mathcal{T}^{\circ} \rightarrow k$-Mod, if two of the functors amongst the $H_{i}$ 's are cohomological, then the third one is cohomological as well. The category of cohomological functors $\mathcal{T}^{\circ} \rightarrow k$-Mod is closed under direct sums.

Given $H_{1} \rightarrow H_{2} \rightarrow \cdots$ a directed system of cohomological functors $\mathcal{T}^{\circ} \rightarrow k$-Mod, we have an exact sequence $0 \rightarrow \bigoplus H_{i} \rightarrow \bigoplus H_{i} \rightarrow \operatorname{colim} H_{i} \rightarrow 0$. This shows that colim $H_{i}$ is a cohomological functor.

Lemma 4.11. Let $H_{1}, \ldots, H_{n+1}$ be cohomological functors on $\mathcal{T}$ and $f_{i}: H_{i} \rightarrow H_{i+1}$ for $1 \leq i \leq n$. Let $\mathcal{I}_{i}$ be a subcategory of $\mathcal{T}$ on which $f_{i}$ vanishes. Then, $f_{n} \cdots f_{1}$ vanishes on $\mathcal{I}_{1} \diamond \cdots \diamond \mathcal{I}_{n}$.

Proof. Note first that if a morphism between cohomological functors vanishes on a subcategory $\mathcal{I}$, then it vanishes on $\langle\mathcal{I}\rangle$.

By induction, it is enough to prove the Lemma for $n=2$. Let $X_{1} \rightarrow X \rightarrow X_{2} \rightsquigarrow$ be a distinguished triangle with $X_{i} \in \mathcal{I}_{i}$. The map $f_{1}(X)$ factors through $H_{2}\left(X_{2}\right)$, i.e., we have a commutative diagram with exact horizontal sequences

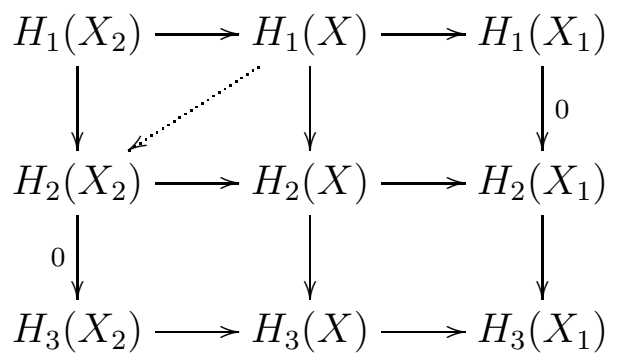

This shows that $f_{2} f_{1}(X)=0$.

Remark 4.12. Let $M \in \mathcal{T}$ be a complete classical generator. Let $f: \bigoplus_{i} \operatorname{Hom}\left(\mathrm{id}_{\mathcal{T}}, \mathrm{id}_{\mathcal{T}}[i]\right) \rightarrow$ $\bigoplus_{i} \operatorname{Hom}(M, M[i])$ be the canonical map. Let $\zeta \in \operatorname{ker} f$. It follows from Lemma 4.11 that $\zeta$ is locally nilpotent. If $\mathcal{T}=\langle\bar{M}\rangle_{d}$, then $(\operatorname{ker} f)^{d}=0$. 
4.2.2. In this part, we study convergence conditions on directed systems. This builds on [BoVdB, $\S 2.3]$.

Let $V_{1} \stackrel{f_{1}}{\longrightarrow} V_{2} \stackrel{f_{2}}{\longrightarrow} \cdots$ be a system of abelian groups. We say that the system $\left(V_{i}\right)$ is almost constant if one of the following equivalent conditions is satisfied :

- $V_{i}=\operatorname{im} f_{i-1} \cdots f_{2} f_{1}+\operatorname{ker} f_{i}$ and $\operatorname{ker} f_{i+r} \cdots f_{i}=\operatorname{ker} f_{i}$ for any $r \geq 0$ and $i \geq 1$.

- Denote by $\alpha_{i}: V_{i} \rightarrow V=\operatorname{colim} V_{i}$ the canonical map. Then, $\alpha_{i}$ induces an isomorphism $V_{i} / \operatorname{ker} f_{i} \stackrel{\sim}{\rightarrow} V$.

Let $\mathcal{T}$ be a triangulated category and $\mathcal{I}$ a subcategory of $\mathcal{T}$. Let $H_{1} \rightarrow H_{2} \rightarrow \cdots$ be a directed system of functors $\mathcal{T}^{\circ} \rightarrow k$-Mod. We say that $\left(H_{i}\right)_{i \geq 1}$ is almost constant on $\mathcal{I}$ if for every $X \in \mathcal{I}$, the system $H_{1}(X) \rightarrow H_{2}(X) \rightarrow \cdots$ is almost constant.

Given $1 \leq r_{1}<r_{2}<\cdots$, we denote by $\left(H_{r_{i}}\right)$ the system $H_{r_{1}} \stackrel{f_{r_{2}-1} \cdots f_{r_{1}+1} f_{r_{1}}}{\longrightarrow} H_{r_{2}} \stackrel{f_{r_{3}-1} \cdots f_{r_{2}+1} f_{r_{2}}}{\longrightarrow}$ $H_{r_{3}} \rightarrow \cdots$.

Proposition 4.13. Let $\left(H_{i}\right)_{i \geq 1}$ be a directed system of cohomological functors on $\mathcal{T}$.

(i) If $\left(H_{i}\right)_{i \geq 1}$ is almost constant on $\mathcal{I}_{1}, \mathcal{I}_{2}, \ldots, \mathcal{I}_{n}$, then, for any $r>0$, the system $\left(H_{n i+r}\right)_{i \geq 0}$ is almost constant on $\mathcal{I}_{1} \diamond \cdots \diamond \mathcal{I}_{n}$.

Assume now $\left(H_{i}\right)_{i \geq 1}$ is almost constant on $\mathcal{I}$. Then,

(ii) $\left(H_{i}\right)_{i \geq 1}$ is almost constant on $\operatorname{add}(\mathcal{I})$. If in addition the functors $H_{i}$ commute with products, then $\left(H_{i}\right)_{i \geq 1}$ is almost constant on $\overline{\operatorname{add}}(\mathcal{I})$.

(iii) $\left(H_{i r+s}\right)_{i \geq 0}$ is almost constant on $\mathcal{I}$ for any $r, s>0$.

(iv) the canonical map $H_{n+1} \rightarrow$ colim $H_{i}$ is a split surjection, when the functors are restricted to $\langle\mathcal{I}\rangle_{n}$.

Proof. Let $H=\operatorname{colim} H_{i}$ and let $K_{i}=\operatorname{ker}\left(H_{i} \rightarrow H\right)$. Take $\mathcal{I}$ and $\mathcal{I}^{\prime}$ such that $\left(H_{i}\right)$ is almost constant on $\mathcal{I}$ and $\mathcal{I}^{\prime}$. Let $I \rightarrow J \rightarrow I^{\prime}$ be a distinguished triangle with $I \in \mathcal{I}$ and $I^{\prime} \in \mathcal{I}^{\prime}$.

Given $i \geq 1$, we have a commutative diagram with exact rows and columns

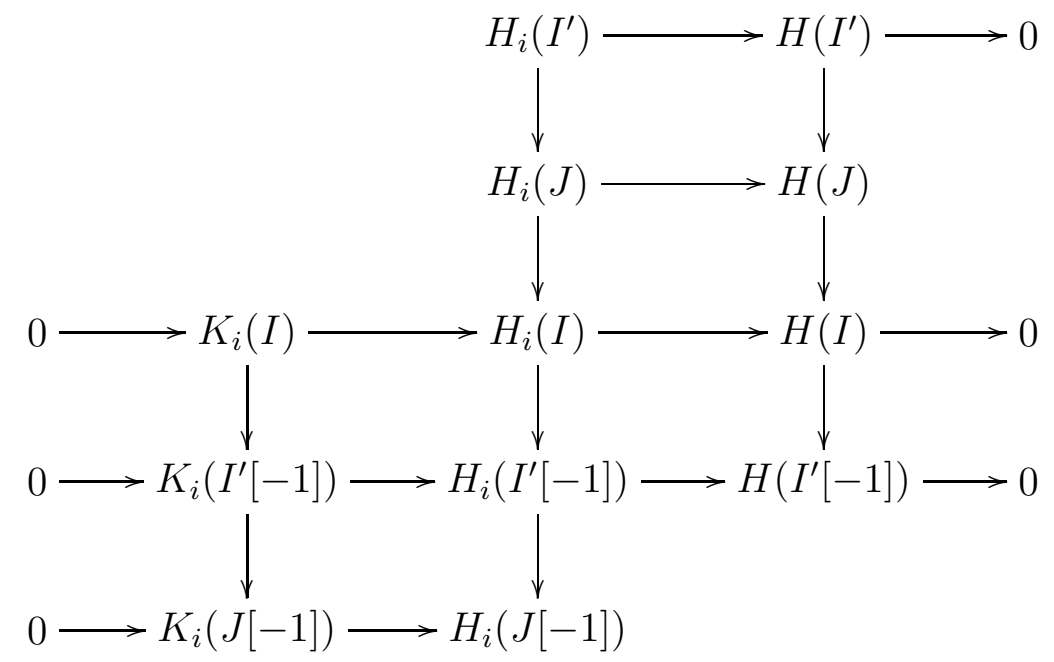

This shows that $H_{i}(J) \rightarrow H(J)$ is onto. By induction, we deduce that $H_{i}(X) \rightarrow H(X)$ is onto for any $i \geq 1$ and any $X \in \mathcal{I}_{1} \diamond \cdots \diamond \mathcal{I}_{n}$. It follows from Lemma 4.11 that the composition $K_{i} \stackrel{f_{i}}{\longrightarrow} K_{i+1} \rightarrow \cdots \rightarrow K_{i+n}$ vanishes on $\mathcal{I}_{1} \diamond \cdots \diamond \mathcal{I}_{n}$. We deduce that (i) holds. 
The assertions (ii) and (iii) are clear.

By (i), it is enough to prove (iv) for $n=1$. The map $f_{1}: H_{1} \rightarrow H_{2}$ factors through $H_{1} / K_{1}$ as $\bar{f}_{1}: H_{1} / K_{1} \rightarrow H_{2}$. We have a commutative diagram

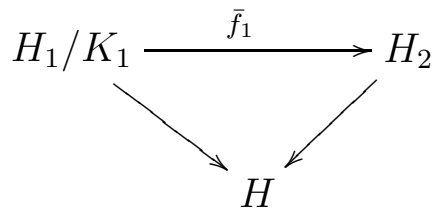

When restricted to $\mathcal{I}$, the canonical map $H_{1} / K_{1} \rightarrow H$ is an isomorphism, hence the canonical map $H_{2} \rightarrow H$ is a split surjection. This proves (iv).

We say that a direct system $\left(A_{1} \stackrel{f_{1}}{\longrightarrow} A_{2} \stackrel{f_{2}}{\longrightarrow} \cdots\right)$ of objects of $\mathcal{T}$ is almost constant on $\mathcal{I}$ if the system $\left(h_{A_{i}}\right)$ is almost constant on $\mathcal{I}$.

4.2.3. We study now approximations of locally finitely presented functors.

Lemma 4.14. Let $\mathcal{T}$ be a triangulated category and $G \in \mathcal{T}$. Let $H$ be a locally finitely presented cohomological functor. Then, there is a directed system $A_{1} \stackrel{f_{1}}{\longrightarrow} A_{2} \stackrel{f_{2}}{\longrightarrow} \cdots$ in $\mathcal{T}$ that is almost constant on $\{G[i]\}_{i \in \mathbf{Z}}$ and a map colim $h_{A_{i}} \rightarrow H$ that is an isomorphism on $\langle G\rangle_{\infty}$.

Proof. Since $H$ is locally finitely presented, there is $A_{1} \in \mathcal{T}$ and $\alpha_{1}: h_{A_{1}} \rightarrow H$ such that $\alpha_{1}(G[r])$ is onto for all $r$.

We now construct the system by induction on $i$. Assume $A_{1} \stackrel{f_{1}}{\longrightarrow} A_{2} \stackrel{f_{2}}{\longrightarrow} \cdots \stackrel{f_{i-1}}{\longrightarrow} A_{i}$ and $\alpha_{1}, \ldots, \alpha_{i}$ have been constructed.

Since $H$ is locally finitely presented, there is $g: B \rightarrow A_{i}$ with $\operatorname{im} h_{g}(G[r])=\operatorname{ker} \alpha_{i}(G[r])$ for all $r$ and with $h_{g} \alpha_{i}=0$. Let $B \stackrel{g}{\longrightarrow} A_{i} \stackrel{f_{i}}{\longrightarrow} A_{i+1} \rightsquigarrow$ be a distinguished triangle. We have an exact sequence $h_{B} \stackrel{h_{g}}{\longrightarrow} h_{A_{i}} \stackrel{h_{f_{i}}}{\longrightarrow} h_{A_{i+1}}$, hence, there is $\alpha_{i+1}: h_{A_{i+1}} \rightarrow H$ with $\alpha_{i}=\alpha_{i+1} f_{i}$. We have a surjection $h_{g}(G[r]): h_{B}(G[i]) \rightarrow \operatorname{ker} \alpha_{i}(G[r])$, hence $\operatorname{ker} \alpha_{i}(G[r]) \subseteq \operatorname{ker} f_{i}(G[r])$. So, the system is almost constant on $\{G[i]\}_{i \in \mathbf{Z}}$. It follows from Proposition 4.13 (iv) that the canonical map $H \rightarrow \operatorname{colim} h_{A_{i}}$ is an isomorphism on $\langle G\rangle_{\infty}$.

Proposition 4.15. Let $\mathcal{T}$ be a triangulated category classically generated by an object $G$. Let $H$ be a cohomological functor. Then, $H$ is locally finitely presented if and only if there is a directed system $A_{1} \stackrel{f_{1}}{\longrightarrow} A_{2} \stackrel{f_{2}}{\longrightarrow} \cdots$ in $\mathcal{T}$ that is almost constant on $\{G[i]\}_{i \in \mathbf{Z}}$ and an isomorphism $\operatorname{colim} h_{A_{i}} \stackrel{\sim}{\rightarrow} H$.

Proof. The first implication is given by Lemma 4.14. Let us now show the converse.

Since $\mathcal{T}$ is classically generated by $G$, it is enough to show conditions (a) and (b) for $X=G$ (cf Lemma 4.6). Condition (a) is obtained with $\alpha_{1}: h_{A_{1}} \rightarrow H$. Fix now $\beta: h_{E} \rightarrow H$. There is an integer $i$ such that $E \in\langle G\rangle_{i}$. By Proposition 4.13 (iii) and (iv), the restriction of $\alpha_{i+1}$ to $\langle G\rangle_{i}$ has a right inverse $\rho$. We obtain a map $\rho \beta$ between the functors $h_{E}$ and $h_{A_{i+1}}$ restricted to $\langle G\rangle_{i}$. It comes from a map $f: E \rightarrow A_{i+1}$. Let $F$ be the cocone of $f$. The kernel of $h_{f}(G[r]): h_{E}(G[r]) \rightarrow h_{A_{i+1}}(G[r])$ is the same as the kernel of $\beta(G[r])$. So, the exact sequence $h_{F}(G[r]) \rightarrow h_{E}(G[r]) \rightarrow h_{A_{i+1}}(G[r])$ induces an exact sequence $h_{F}(G[r]) \rightarrow$ $h_{E}(G[r]) \rightarrow H(G[r])$ and (b) is satisfied. 


\subsection{Representability.}

4.3.1. We can now state a representability Theorem for strongly finitely generated triangulated categories.

Theorem 4.16. Let $\mathcal{T}$ be a strongly finitely generated triangulated category and $H$ be a cohomological functor.

Then, $H$ is locally finitely presented if and only if it is a direct summand of a representable functor.

Proof. Let $G$ be a $d$-step generator of $\mathcal{T}$ for some $d \in \mathbf{N}$. Let $\left(A_{i}\right)$ be a directed system as in Lemma 4.14. Then, $\alpha_{d+1}: h_{A_{d+1}} \rightarrow H$ is a split surjection by Proposition 4.13 (iv). The converse follows from Lemmas 4.2 and 4.6 .

Recall that an additive category is Karoubian if for every object $X$ and every idempotent $e \in \operatorname{End}(X)$, there is an object $Y$ and maps $i: Y \rightarrow X$ and $p: X \rightarrow Y$ such that $p i=\mathrm{id}_{Y}$ and $i p=e$.

Corollary 4.17. Let $\mathcal{T}$ be a strongly finitely generated Karoubian triangulated category. Then, every locally finitely presented cohomological functor is representable.

Via Proposition 4.9, Theorem 4.16 generalizes the following result of Bondal and Van den Bergh [BoVdB, Theorem 1.3].

Corollary 4.18. Let $\mathcal{T}$ be an Ext-finite strongly finitely generated Karoubian triangulated category. A cohomological functor $H: \mathcal{T}^{\circ} \rightarrow k$-Mod is representable if and only if it is locally finite.

The following Lemma is classical:

Lemma 4.19. Let $\mathcal{T}$ be a triangulated category closed under countable multiples. Then, $\mathcal{T}$ is Karoubian.

Proof. Given $X \in \mathcal{T}$ and $e \in \operatorname{End}(X)$ an idempotent, then $\operatorname{hocolim}(X \stackrel{e}{\rightarrow} X \stackrel{e}{\rightarrow} X \rightarrow \cdots)$ is the image of $e$.

We have a variant of Theorem 4.16, with a similar proof :

Theorem 4.20. Let $\mathcal{T}$ be a triangulated category that has a strong complete generator and $H$ be a cohomological functor that commutes with products.

Then, $H$ is locally finitely presented if and only if it is a direct summand of a representable functor.

If $\mathcal{T}$ is closed under countable multiples, then $H$ is locally finitely presented if and only if it is representable.

4.3.2. Let us now consider cocomplete and compactly generated triangulated categories — the "classical" setting.

Lemma 4.21. Assume $\mathcal{T}$ is cocomplete. Then, every functor is locally finitely presented.

Proof. Let $H$ be a functor and $X \in \mathcal{T}$. Let $D=\bigoplus_{i} X[i]^{|H(X[i])|}$ and $\alpha: h_{D} \rightarrow H$ the canonical map. Then, $\alpha(X[i])$ is surjective for every $i$. It follows that $H$ is locally finitely generated.

Now, the kernel of a map $h_{E} \rightarrow H$ will also be locally finitely generated, hence $H$ is locally finitely presented. 
So, we can derive the classical representability Theorem ([Nee3, Theorem 3.1], [Ke, Theorem 5.2], [Nee2, Lemma 2.2]) :

Theorem 4.22. Let $\mathcal{T}$ be a cocomplete triangulated category generated by a set $\mathcal{S}$ of compact objects. Then,

(1) a cohomological functor $\mathcal{T}^{\circ} \rightarrow k$-Mod is representable if and only if it commutes with products

(2) every object of $\mathcal{T}$ is a homotopy colimit of a system $A_{1} \stackrel{f_{1}}{\longrightarrow} A_{2} \stackrel{f_{2}}{\longrightarrow} \cdots$ almost constant on $\langle\overline{\mathcal{S}}\rangle$ and such that $A_{1}$ and the cone of $f_{i}$ for all $i$ are in $\overline{\mathcal{S}}$. In particular, $\mathcal{T}$ is the smallest full triangulated subcategory containing $\mathcal{S}$ and closed under direct sums.

(3) $\mathcal{S}$ classically generates $\mathcal{T}^{c}$.

Proof. Let $G=\bigoplus_{S \in \mathcal{S}} S$. Let $H: \mathcal{T}^{\circ} \rightarrow k$-Mod be a cohomological functor that commutes with products. Let $\left(A_{i}, f_{i}\right)$ be a directed system constructed as in Lemma 4.14 and $C=\operatorname{hocolim} A_{i}$. Note that we can assume that $A_{1}$ and the cone of $f_{i}$ are direct sums of shifts of $G$ (cf Lemmas 4.14 and 4.21). By Proposition 4.13 (ii), the system is almost constant on $\langle\overline{\mathcal{S}}\rangle$.

The distinguished triangle $\bigoplus A_{i} \rightarrow \bigoplus A_{i} \rightarrow C \rightsquigarrow$ induces an exact sequence $H(C) \rightarrow$ $\prod H\left(A_{i}\right) \rightarrow \prod H\left(A_{i}\right)$, since $H$ takes direct sums in $\mathcal{T}$ to products. Consequently, there is a map $f: h_{C} \rightarrow H$ that makes the following diagram commutative

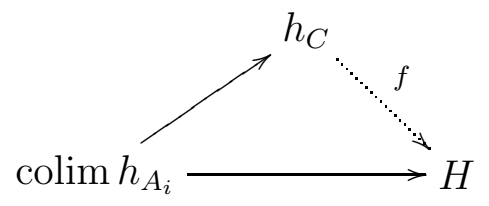

where the canonical maps from colim $h_{A_{i}}$ are isomorphisms when the functors are restricted to $\langle\mathcal{S}\rangle$ (cf Lemma 3.11). So, the restriction of $f$ to $\langle\mathcal{S}\rangle$ is an isomorphism. Consequently, $f$ is an isomorphism on the smallest full triangulated subcategory $\mathcal{T}^{\prime}$ of $\mathcal{T}$ containing $\mathcal{S}$ and closed under direct sums. To conclude, it is enough to show that $\mathcal{T}^{\prime}=\mathcal{T}$ and we will prove the more precise assertion (2) of the Theorem.

We take $X \in \mathcal{T}$ and $H=h_{X}$. Then, $f$ comes from a map $g: C \rightarrow X$. The cone $Y$ of $g$ is zero, since $\operatorname{Hom}(S[i], Y)=0$ for all $S \in \mathcal{S}$ and $i \in \mathbf{Z}$. Hence, $g$ is an isomorphism, so (2) holds.

Assume finally that $X \in \mathcal{T}^{c}$. Then, $g^{-1}: X \stackrel{\sim}{\rightarrow} C$ factors through some object of $\langle\mathcal{S}\rangle_{i}$ by Proposition 3.12, hence $X \in\langle\mathcal{S}\rangle_{i}$.

4.3.3. We deduce a general duality property for compact objects.

Corollary 4.23. Let $\mathcal{T}$ be a cocomplete compactly generated triangulated category over a field $k$. Then, there is a faithful functor $S: \mathcal{T}^{c} \rightarrow \mathcal{T}$ and bifunctorial isomorphisms

$$
\operatorname{Hom}(C, D)^{*} \stackrel{\sim}{\rightarrow} \operatorname{Hom}(D, S(C))
$$

for $C \in T^{c}$ and $D \in \mathcal{T}$. If $\operatorname{Hom}(C, D)$ is finite dimensional for all $C, D \in \mathcal{T}^{c}$, then $S$ is fully faithful.

Proof. Let $C \in \mathcal{T}^{c}$. The cohomological functor $\operatorname{Hom}(C,-)^{*}: \mathcal{T}^{\circ} \rightarrow k$-Mod commutes with products, hence it is representable by an object $S(C) \in \mathcal{T}$ by Theorem 4.22. By Yoneda's Lemma, this defines a functor $S: \mathcal{T}^{c} \rightarrow \mathcal{T}$. Now, if $D \in \mathcal{T}^{c}$, then $S$ is equal to the composition

$$
\operatorname{Hom}(C, D) \stackrel{\text { can }}{\longrightarrow} \operatorname{Hom}(C, D)^{* *} \stackrel{\sim}{\rightarrow} \operatorname{Hom}(D, S(C))^{*} \stackrel{\sim}{\rightarrow} \operatorname{Hom}(S(C), S(D)) .
$$


Whenever $\mathcal{T}^{c}$ admits a Serre functor, it must be the restriction of the $S$ above.

Corollary 4.24. Let $\mathcal{T}$ be a cocomplete compactly generated triangulated category over a field $k$. Assume there is a self-equivalence $S^{\prime}$ of $\mathcal{T}^{c}$ together with bifunctorial isomorphisms

$$
\operatorname{Hom}(C, D)^{*} \stackrel{\sim}{\rightarrow} \operatorname{Hom}\left(D, S^{\prime}(C)\right)
$$

for $C, D \in T^{c}$. Then, $S$ takes values in $\mathcal{T}^{c}$ and there is a unique isomorphism $S^{\prime} \stackrel{\sim}{\rightarrow} S$ making the following diagram commutative for any $C, D \in \mathcal{T}^{c}$

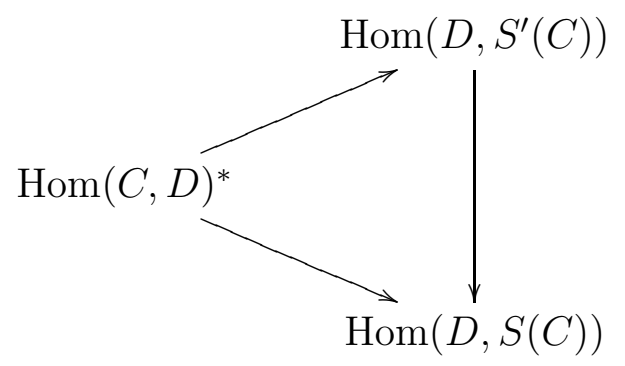

Proof. A bifunctorial isomorphism $\operatorname{Hom}\left(D, S^{\prime}(C)\right) \stackrel{\sim}{\rightarrow} \operatorname{Hom}(D, S(C))$ comes from a unique functorial map $S^{\prime}(C) \rightarrow S(C)$. Its cone is right orthogonal to $\mathcal{T}^{c}$, hence it is zero, since $\mathcal{T}$ is generated by $\mathcal{T}^{c}$.

\subsection{Finiteness for objects.}

4.4.1. We say that $C$ is cohomologically locally bounded (resp. bounded above, resp. bounded below, resp. finitely generated, resp. finitely presented, resp. finite) if the restriction of $h_{C}$ to $\mathcal{T}^{c}$ has that property.

From Lemma 4.2, we deduce

Lemma 4.25. Let $C \in \mathcal{T}^{c}$. Then, $C$ is cohomologically locally finitely presented.

Lemma 4.26. Let $C \in \mathcal{T}$ be cohomologically locally finitely generated. Then, $C$ is cohomologically locally finitely presented if and only if given $X \in \mathcal{T}^{c}, E \in \mathcal{T}^{c}$ and $\beta: E \rightarrow C$ such that $\operatorname{Hom}(X[i], \beta)$ is surjective for every $i \in \mathbf{Z}$, then the cocone of $\beta$ is cohomologically locally finitely generated.

Proof. Let $F$ be the cocone of $\beta$. We have an exact sequence

$$
0 \rightarrow \operatorname{Hom}(X[i], F) \rightarrow \operatorname{Hom}(X[i], E) \stackrel{\operatorname{Hom}(X[i], \beta)}{\longrightarrow} \operatorname{Hom}(X[i], C) \rightarrow 0 .
$$

The Lemma follows now from Lemma 4.4 .

From Lemma 4.14 and Proposition 4.13 (ii), we obtain

Lemma 4.27. Assume $\mathcal{T}$ is cocomplete and generated by a compact object $G$. Let $C \in \mathcal{T}$. Let $C$ be a cohomologically locally finitely presented object of $\mathcal{T}$. Then, there is a system $A_{1} \rightarrow$ $A_{2} \rightarrow \cdots$ in $\mathcal{T}^{c}$ which is almost constant for $\left\langle\overline{\{G[i]\}_{i \in \mathbf{Z}}}\right\rangle$ and an isomorphism hocolim $A_{i} \stackrel{\sim}{\rightarrow} C$.

In particular, given $d \geq 0$, there is $D \in \mathcal{T}^{c}$ and $f: D \rightarrow C$ such that every map from an object of $\left\langle\overline{\left.\{G[i]\}_{i \in \mathbf{Z}}\right\rangle_{d}}\right.$ to $C$ factors through $f$.

From Propositions 4.3 and 4.10, we deduce 
Proposition 4.28. The full subcategory of $\mathcal{T}$ of cohomologically locally finitely presented (resp. bounded) objects is a thick subcategory.

Note that the full subcategory of cohomologically locally bounded (resp. bounded above, resp. bounded below) objects is also a thick subcategory.

From Theorem 4.16, we deduce

Corollary 4.29. Let $\mathcal{T}$ be a triangulated category such that $\mathcal{T}^{c}$ is strongly finitely generated. Then, $C \in \mathcal{T}^{c}$ if and only if $C$ is cohomologically locally finitely presented.

Remark 4.30. Not all cohomological functors on $\mathcal{T}^{c}$ are isomorphic to the restriction of $h_{C}$, for some $C \in \mathcal{T}$. This question has been studied for example in [Nee4, Bel, ChKeNea]. Let us mention the following result [ChKeNee, Lemma 2.13] : let $\mathcal{T}$ be a cocomplete and compactly generated triangulated category. Assume $k$ is a field. Let $H$ be a cohomological functor on $\mathcal{T}^{c}$ with value in the category $k$-mod of finite dimensional vector spaces. Then there is $C \in \mathcal{T}$ such that $H$ is isomorphic to the restriction of $h_{C}$ to $\mathcal{T}^{c}$.

\section{LOCALIZATION}

\subsection{Compact objects.}

5.1.1. Let us recall Thomason's classification of dense subcategories [Th, Theorem 2.1] :

Theorem 5.1. Let $\mathcal{T}$ be a triangulated category and $\mathcal{I}$ a dense full triangulated subcategory. Then, an object of $\mathcal{T}$ is isomorphic to an object of $\mathcal{I}$ if and only if its class is in the image of the canonical map $K_{0}(\mathcal{I}) \rightarrow K_{0}(\mathcal{T})$.

The following Lemma is proved in [BöNee, Lemma 1.5].

Lemma 5.2. Let $\mathcal{T}$ be a cocomplete triangulated category and $\mathcal{I}$ be a thick subcategory closed under direct sums. Then, $\mathcal{T} / \mathcal{I}$ is cocomplete and the quotient functor $\mathcal{T} \rightarrow \mathcal{T} / \mathcal{I}$ commutes with direct sums.

The following is a version of Thomason-Trobaugh-Neeman's Theorem [Nee2, Theorem 2.1].

Theorem 5.3. Let $\mathcal{T}$ be a cocomplete and compactly generated triangulated category. Let $\mathcal{I}$ a full triangulated subcategory closed under direct sums and compactly generated in $\mathcal{T}$. Denote by $F: \mathcal{T} \rightarrow \mathcal{T} / \mathcal{I}$ the quotient functor. Then,

(i) $\mathcal{I}$ is a cocomplete compactly generated triangulated category and $\mathcal{I}^{c}=\mathcal{I} \cap \mathcal{T}^{c}$.

(ii) Given $X \in \mathcal{T}^{c}$ and $Y \in \mathcal{T}$, the canonical map

$$
\lim \operatorname{Hom}_{\mathcal{T}}\left(X^{\prime}, Y\right) \stackrel{\sim}{\rightarrow} \operatorname{Hom}_{\mathcal{T} / \mathcal{I}}(F X, F Y)
$$

is an isomorphism, where the limit is taken over the maps $X^{\prime} \rightarrow X$ whose cone is in $\mathcal{I}^{c}$. Also, if $F Y$ is in $F\left(\mathcal{T}^{c}\right)$, then, there is $C \in \mathcal{T}^{c}$ and $f: C \rightarrow Y$ such that $F(f)$ is an isomorphism.

(iii) $F$ commutes with direct sums and the canonical functor $\mathcal{T}^{c} / \mathcal{I}^{c} \rightarrow \mathcal{T} / \mathcal{I}$ factors through a fully faithful functor $G: \mathcal{T}^{c} / \mathcal{I}^{c} \rightarrow(\mathcal{T} / \mathcal{I})^{c}$.

(iv) An object of $(\mathcal{T} / \mathcal{I})^{c}$ is isomorphic to an object in the image of $G$ if and only if its class is in the image of $K_{0}(G)$. 
Proof. It is clear that $\mathcal{I}$ is cocomplete and that $\mathcal{T}^{c} \cap \mathcal{I} \subset \mathcal{I}^{c}$. Let $\mathcal{S}_{\mathcal{I}}$ be a set of objects of $\mathcal{T}^{c} \cap \mathcal{I}$ that generates $\mathcal{I}$. It follows from Theorem 4.22 (3) that $\mathcal{S}_{\mathcal{I}}$ classically generates $\mathcal{I}^{c}$. Since $\mathcal{T}^{c} \cap \mathcal{I}$ is a thick subcategory of $\mathcal{I}$, it follows that $\mathcal{I}^{c}=\mathcal{T} \cap \mathcal{I}^{c}$ and (i) is proven.

Let $X \in \mathcal{T}^{c}$ and $Y \in \mathcal{T}$. Let $\phi: W \rightarrow X$ and $\psi: W \rightarrow Y$ with $W \in \mathcal{T}$. Let $Z$ be a cone of $\phi$ and assume $Z \in \mathcal{I}$. By Theorem 4.22 (2) and Proposition 3.12, $X \rightarrow Z$ factors through a map $\alpha: X \rightarrow Z^{\prime}$ for some $Z^{\prime} \in \mathcal{I} \cap \mathcal{T}^{c}$. Let $X^{\prime}$ be the cocone of $\alpha$. The map $X^{\prime} \rightarrow X$ factors as a composition $\phi \zeta$. This shows (ii).

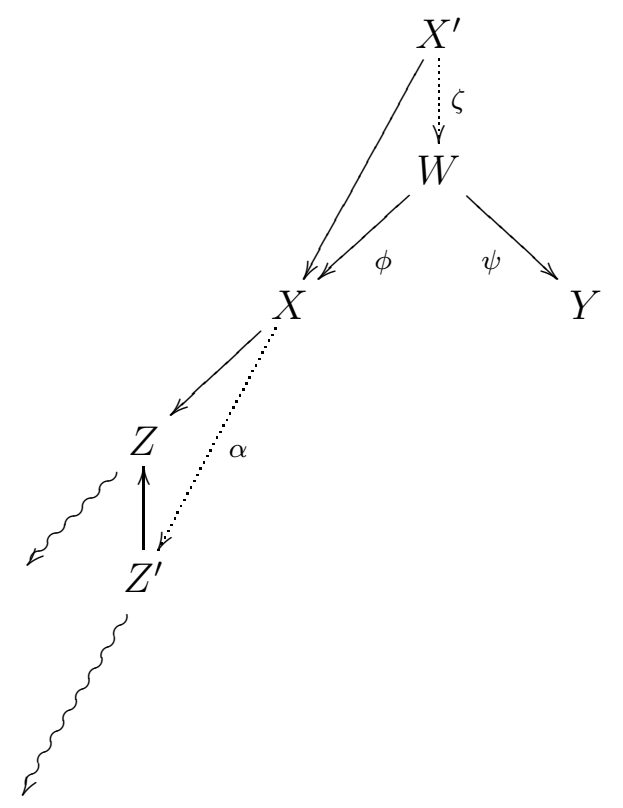

Since $\mathcal{T}$ is cocomplete and the direct sum in $\mathcal{T}$ of objects of $\mathcal{I}$ is in $\mathcal{I}$, it follows from Lemma 5.2 that $F$ commutes with direct sums.

Let now $X \in \mathcal{T}^{c}$ and $\left\{Z_{i}\right\}$ be a family of elements of $\mathcal{T}$. Let $f: F(X) \rightarrow \bigoplus_{i} F\left(Z_{i}\right)=$ $F\left(\bigoplus_{i} Z_{i}\right)$. There is $\phi: X^{\prime} \rightarrow X$ and $\psi: X^{\prime} \rightarrow \bigoplus_{i} Z_{i}$ with the cone of $\phi$ in $\mathcal{I} \cap \mathcal{T}^{c}$ and $f=F(\psi) F(\phi)^{-1}$. Since $X^{\prime}$ is compact, $\psi$ factors through a finite sum of $Z_{i}$ 's, hence $f$ factors through a finite sum of $F\left(Z_{i}\right)$ 's. Consequently, $F(X)$ is compact. The fully faithfulness of $G$ comes from (ii).

Let us now prove (iv). By Theorem $4.22(3),(\mathcal{T} / \mathcal{I})^{c}$ is classically generated by $F\left(\mathcal{T}^{c}\right)$. Since $F\left(\mathcal{T}^{c}\right)$ is a full triangulated subcategory of $(\mathcal{T} / \mathcal{I})^{c}$, it is dense. The result follows now from Theorem 5.1.

Corollary 5.4. Let $\mathcal{T}$ be a cocomplete and compactly generated triangulated category. Let $\mathcal{I}$ be a full triangulated subcategory closed under direct sums and generated by an object $G \in \mathcal{T}^{c} \cap \mathcal{I}$ such that for all $C \in \mathcal{T}^{c}$, then $\operatorname{Hom}(C, G[i])=0$ for $|i| \gg 0$.

If $\mathcal{T}^{c}$ is strongly finitely generated, then $(\mathcal{T} / \mathcal{I})^{c}$ is strongly finitely generated.

Remark 5.5. Let $\mathcal{T}$ be a cocomplete triangulated category generated by a set $\mathcal{E}$ of compact objects and let $\mathcal{I}$ a thick subcategory closed under direct sums. If the inclusion functor $\mathcal{I} \rightarrow \mathcal{T}$ has a left adjoint $G$, then $G(\mathcal{E})$ is a generating set for $\mathcal{I}$ and it consists of compact objects of $\mathcal{T}$.

\subsection{Proper intersections of Bousfield subcategories.}


5.2.1. Let $\mathcal{T}$ be a triangulated category and $\mathcal{I}$ be a thick subcategory. We have a canonical fully faithful functor $i_{*}: \mathcal{I} \rightarrow \mathcal{T}$ and a canonical essentially surjective quotient functor $j^{*}: \mathcal{T} \rightarrow \mathcal{T} / \mathcal{I}$. We say that there is an exact sequence of triangulated categories

$$
0 \rightarrow \mathcal{I} \stackrel{i_{*}}{\rightarrow} \mathcal{T} \stackrel{j^{*}}{\rightarrow} \mathcal{T} / \mathcal{I} \rightarrow 0
$$

We say that $C \in \mathcal{T}$ is $\mathcal{I}$-local if $\operatorname{Hom}(M, C)=0$ for all $M \in \mathcal{I}$. Note that given $C, D \in \mathcal{T}$ with $D$ an $\mathcal{I}$-local object, then $\operatorname{Hom}(C, D) \stackrel{\sim}{\rightarrow} \operatorname{Hom}\left(j^{*} C, j^{*} D\right)$.

Let $\mathcal{I}^{\prime}$ be a thick subcategory of $\mathcal{I}$. Then, we have a commutative diagram of exact sequences of triangulated categories

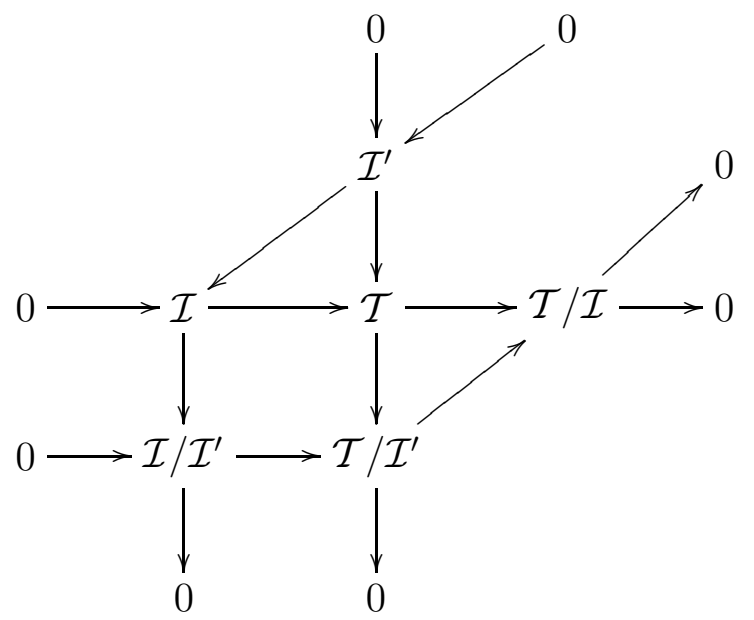

5.2.2. Let us recall the construction of Bousfield localization (cf e.g. [Nee5, §9.1]).

We say that $\mathcal{I}$ is a Bousfield subcategory if the quotient functor $j^{*}: \mathcal{I} \rightarrow \mathcal{T} / \mathcal{I}$ has a right adjoint $j_{*}$. We then denote by $\eta: \mathrm{id}_{\mathcal{T}} \rightarrow j_{*} j^{*}$ the corresponding unit.

Assume $\mathcal{I}$ is a Bousfield subcategory. Note that $C$ is $\mathcal{I}$-local if and only if $\eta(C): C \rightarrow j_{*} j^{*} C$ is an isomorphism if and only if $C \simeq j_{*} C^{\prime}$ for some $C^{\prime} \in \mathcal{T} / \mathcal{I}$.

We denote by $i_{*}: \mathcal{I} \rightarrow \mathcal{T}$ the inclusion functor. Let $C \in \mathcal{T}$ and $C^{\prime}$ be the cocone of $\eta(C)$. We have $j^{*} C^{\prime}=0$, hence $C^{\prime} \in \mathcal{I}$. Since $j_{*} j^{*} C[-1]$ is $\mathcal{I}$-local, the object $C^{\prime}$ is well defined up to unique isomorphism. So, there is a functor $i^{!}: \mathcal{T} \rightarrow \mathcal{I}$ and a map $\varepsilon: i_{*} i^{!} \rightarrow \mathrm{id}_{\mathcal{T}}$ such that the following triangle is distinguished

$$
i_{*} i \stackrel{\varepsilon}{\rightarrow} \operatorname{id}_{\mathcal{T}} \stackrel{\eta}{\rightarrow} j_{*} j^{*} \rightsquigarrow .
$$

Furthermore, $\varepsilon$ provides $\left(i_{*}, i^{!}\right)$with the structure of an adjoint pair.

Since $i_{*}$ and $j^{*}$ have right adjoints, they commute with direct sums. Also, $\mathcal{I}$ is closed under direct sums (taken in $\mathcal{T}$ ) and we have $i^{!} j_{*}=j^{*} i_{*}=0$. The unit of adjunction $\operatorname{id}_{\mathcal{I}} \stackrel{\sim}{\rightarrow} i^{!} i_{*}$ is an isomorphism, as well as the counit $j^{*} j_{*} \stackrel{\sim}{\rightarrow} \mathrm{id}_{\mathcal{T} / \mathcal{I}}$.

Let $\mathcal{I}$ be a thick subcategory of $\mathcal{T}$. Then, the following conditions are equivalent

- $\mathcal{I}$ is a Bousfield subcategory

- for any $C \in \mathcal{T}$, there is a distinguished triangle $C_{1} \rightarrow C \rightarrow C_{2} \rightsquigarrow$ with $C_{1} \in \mathcal{I}$ and $C_{2}$ an $\mathcal{I}$-local object.

- the restriction of $j^{*}$ to the full subcategory of $\mathcal{I}$-local objects is an equivalence. 
Let $\mathcal{I}^{\prime}$ be a Bousfield subcategory of $\mathcal{T}$ containing $\mathcal{I}$. Then, $\mathcal{I}$ is a Bousfield subcategory of $\mathcal{I}^{\prime}$. The right adjoint to the inclusion of $\mathcal{I}$ in $\mathcal{I}^{\prime}$ is $i^{\prime} i_{*}^{\prime}$. Also, $\mathcal{I}^{\prime} / \mathcal{I}$ is a Bousfield subcategory of $\mathcal{T} / \mathcal{I}$ and the left adjoint to the quotient $\mathcal{T} / \mathcal{I} \rightarrow \mathcal{T} / \mathcal{I}^{\prime}$ is $j^{*} j_{*}^{\prime}$.

Assume $\mathcal{T}$ is cocomplete and compactly generated and $\mathcal{I}$ is a full triangulated subcategory closed under direct sums. Then, $\mathcal{I}$ is a Bousfield subcategory $\mathbb{N}$ ee5, Example 8.4.5]. Indeed, given $D \in \mathcal{T} / \mathcal{I}$, the functor $\operatorname{Hom}\left(j^{*}(-), D\right): \mathcal{T}^{\circ} \rightarrow k$-Mod is cohomological and commutes with products (Theorem 5.3), hence is representable by Theorem 4.22. The thickness follows from Lemma 4.19.

Remark 5.6. Let $\mathcal{T}$ be a cocomplete compactly generated triangulated category and $\mathcal{I}$ a Bousfield subcategory. If $C \in \mathcal{T}$ is cohomologically locally bounded, then $i^{!} C$ is cohomologically bounded. An object $C^{\prime} \in \mathcal{T} / \mathcal{I}$ is cohomologically locally bounded if and only if $j_{*} C^{\prime}$ is cohomologically locally bounded.

5.2.3. Let $\mathcal{I}_{1}$ and $\mathcal{I}_{2}$ be two Bousfield subcategories of $\mathcal{T}$.

Lemma 5.7. The following assertions are equivalent

(1) $i_{1 *} i_{1}^{!}\left(\mathcal{I}_{2}\right) \subset \mathcal{I}_{2}$ and $i_{2 *} i_{2}^{!}\left(\mathcal{I}_{1}\right) \subset \mathcal{I}_{1}$

(2) $j_{1 *} j_{1}^{*}\left(\mathcal{I}_{2}\right) \subset \mathcal{I}_{2}$ and $j_{2 *} j_{2}^{*}\left(\mathcal{I}_{1}\right) \subset \mathcal{I}_{1}$

(3) the canonical functor $\mathcal{I}_{1} /\left(\mathcal{I}_{1} \cap \mathcal{I}_{2}\right) \oplus \mathcal{I}_{2} /\left(\mathcal{I}_{1} \cap \mathcal{I}_{2}\right) \rightarrow \mathcal{T} /\left(\mathcal{I}_{1} \cap \mathcal{I}_{2}\right)$ is fully faithful

(4) given $M_{1} \in \mathcal{I}_{1}$ and $M_{2} \in \mathcal{I}_{2}$, every map $M_{1} \rightarrow M_{2}$ and every map $M_{2} \rightarrow M_{1}$ factors through an object of $\mathcal{I}_{1} \cap \mathcal{I}_{2}$.

Proof. Given $N \in \mathcal{I}_{2}$, we have a distinguished triangle $i_{1 *} i_{1}^{!} N \rightarrow N \rightarrow j_{1 *} j_{1}^{*} N \rightsquigarrow$. This shows immediately the equivalence between (1) and (2).

Let $f: M \rightarrow N$ with $M \in \mathcal{I}_{1}$. Then, there is $g: M \rightarrow i_{1 *} i_{1}^{!} N$ such that $f=\eta_{1}(N) g$. It is now clear that $(1) \Rightarrow(4)$. Assume (4). Then, there is $L \in \mathcal{I}_{1} \cap \mathcal{I}_{2}$ and $\phi: i_{1 *} i_{1}^{!} N \rightarrow L$ and $\psi: L \rightarrow N$ such that $\varepsilon(N)=\psi \phi$. Now, there is $\phi^{\prime}: L \rightarrow i_{1 *} i_{1}^{!} N$ such that $\psi=\varepsilon(N) \phi^{\prime}$. So, $\varepsilon(N)\left(1-\phi^{\prime} \phi\right)=0$. Since the canonical map $\operatorname{End}\left(i_{1 *} i_{1}^{!} N\right) \rightarrow \operatorname{Hom}\left(i_{1 *} i_{1}^{!} N, N\right), h \mapsto \varepsilon(N) h$ is injective, it follows that $i_{1 *} i_{1}^{!} N$ is a direct of $L$, hence $i_{1 *} i_{1}^{!} N \in \mathcal{I}_{2}$. So, (4) $\Rightarrow(1)$.

A map in $\mathcal{T}$ factors through an object of $\mathcal{I}_{1} \cap \mathcal{I}_{2}$ if and only if it becomes 0 in $\mathcal{T} /\left(\mathcal{I}_{1} \cap \mathcal{I}_{2}\right)$. This shows the equivalence of (3) and (4).

We say that $\mathcal{I}_{1}$ and $\mathcal{I}_{2}$ intersect properly if the assertions of Lemma 5.7 are satisfied. This property passes to intersections, unions, quotients... A collection of Bousfield subcategories any two of which intersect properly behaves like a collection of closed subsets.

We will identify $\mathcal{I}_{1} /\left(\mathcal{I}_{1} \cap \mathcal{I}_{2}\right)$ with its essential image in $\mathcal{T} / \mathcal{I}_{2}$.

There are commutative diagrams of inclusions of subcategories and of quotients of categories
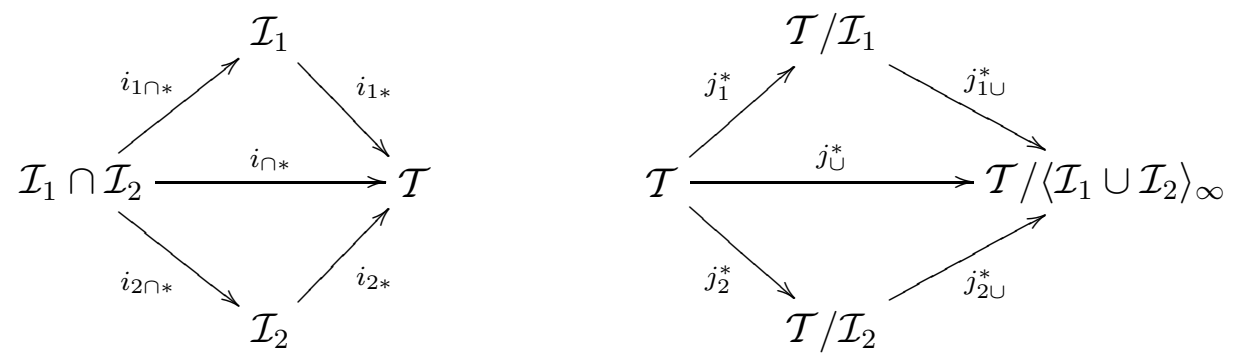

Lemma 5.8. Assume $\mathcal{I}_{1}$ and $\mathcal{I}_{2}$ intersect properly. Let $\{a, b\}=\{1,2\}$. Then, 
- $\mathcal{I}_{1} \cap \mathcal{I}_{2}$ and $\left\langle\mathcal{I}_{1} \cup \mathcal{I}_{2}\right\rangle_{\infty}$ are Bousfield subcategories of $\mathcal{T}$.

- We have $i_{\cap *} i_{\cap}^{!} \simeq i_{a *} i_{a}^{!} i_{b *} i_{b}^{!}$and $j_{\cup *} j_{\cup}^{*} \simeq j_{a *} j_{a}^{*} j_{b *} j_{b}^{*}$.

- There are commutative diagrams
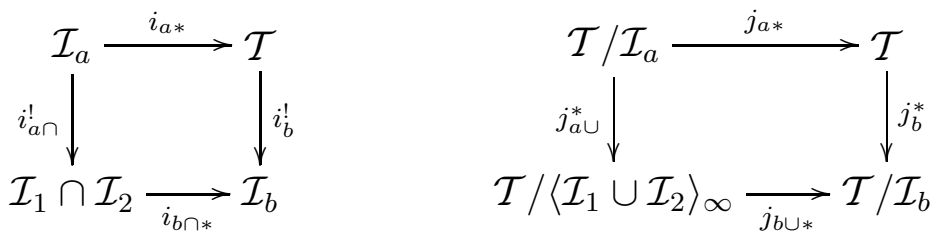

- The canonical functor $\mathcal{I}_{a} /\left(\mathcal{I}_{1} \cap \mathcal{I}_{2}\right) \stackrel{\sim}{\rightarrow}\left\langle\mathcal{I}_{1} \cup \mathcal{I}_{2}\right\rangle_{\infty} / \mathcal{I}_{b}$ is an equivalence and we have a commutative diagram of exact sequences of triangulated categories

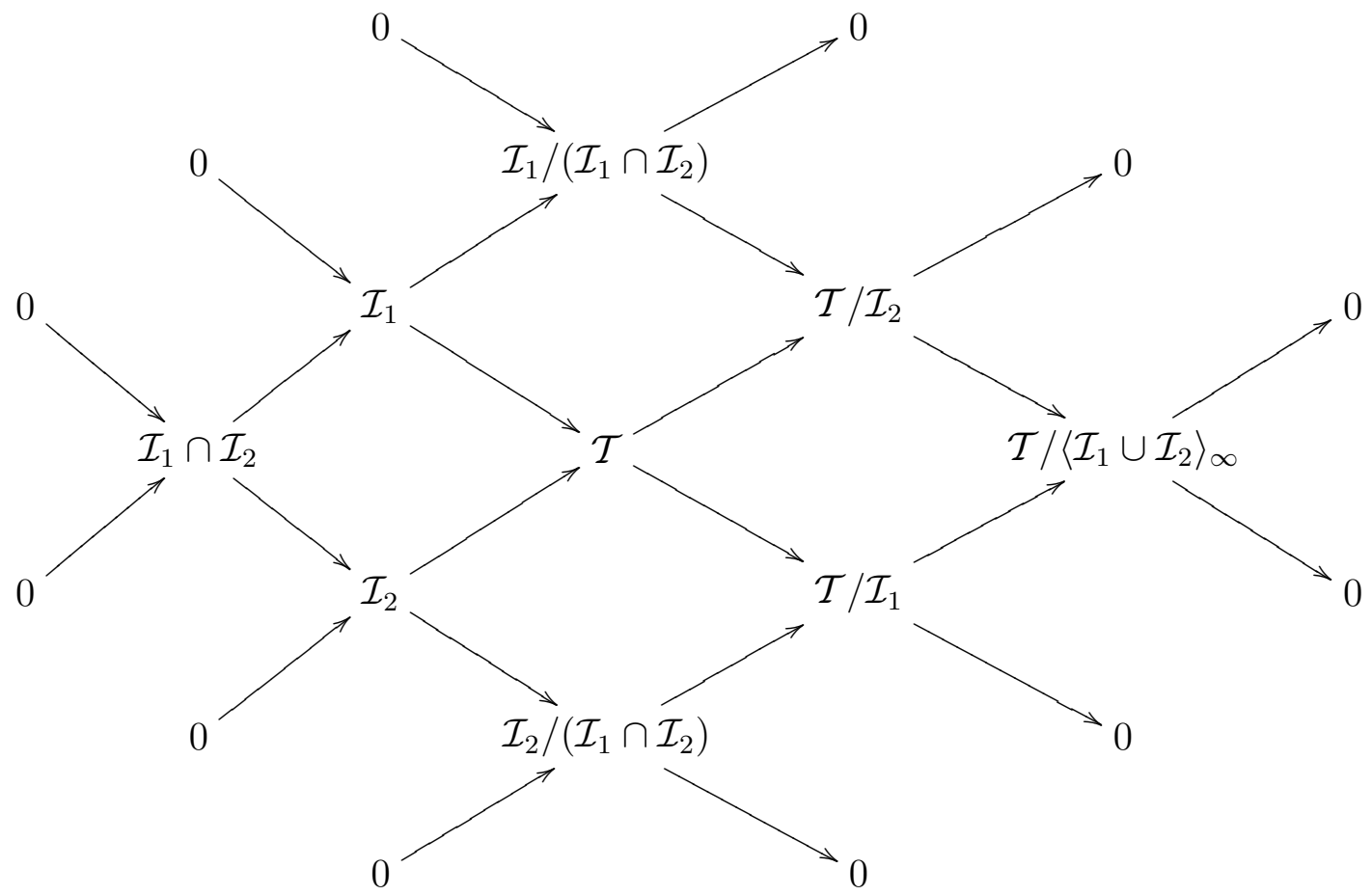

Proof. Let $C \in \mathcal{T}$. We have distinguished triangles

$$
i_{1 *} i_{1}^{!} C \rightarrow C \rightarrow j_{1 *} j_{1}^{*} C \rightsquigarrow \text { and } i_{2 *} i_{2}^{!} i_{1 *} i_{1}^{!} C \rightarrow i_{1 *} i_{1}^{!} C \rightarrow j_{2 *} j_{2}^{*} i_{1 *} i_{1}^{!} C \rightsquigarrow
$$

The octahedral axiom shows that there are $C^{\prime} \in \mathcal{T}$ and distinguished triangles

$$
i_{2 *} i_{2}^{!} i_{1 *} i_{1}^{!} C \rightarrow C \rightarrow C^{\prime} \rightsquigarrow \text { and } j_{2 *} j_{2}^{!} i_{1 *} i_{1}^{!} C \rightarrow C^{\prime} \rightarrow j_{1 *} j_{1}^{*} C \rightsquigarrow
$$

Since $C^{\prime}$ is $\left(\mathcal{I}_{1} \cap \mathcal{I}_{2}\right)$-local and $i_{2 *} i_{2}^{!} i_{1 *} i_{1}^{!} C \in \mathcal{I}_{1} \cap \mathcal{I}_{2}$, we deduce that $\mathcal{I}_{1} \cap \mathcal{I}_{2}$ is a Bousfield subcategory of $\mathcal{T}$. The map $i_{2 *} i_{2} i_{1 *} i_{1}^{!} C \rightarrow C$ factors uniquely through the canonical map $i_{\cap *} i_{\cap}^{!} C \rightarrow C$ and similarly the map $i_{\cap *} i_{\cap}^{!} C \rightarrow C$ factors uniquely through $i_{2 *} i_{2}^{!} i_{1 *} i_{1}^{!} C \rightarrow C$ and this provides functorial inverse morphisms between $i_{\cap *} i_{\cap}^{!} C$ and $i_{2 *} i_{2}^{!} i_{1 *} i_{1}^{!} C$.

The case of $\left\langle\mathcal{I}_{1} \cup \mathcal{I}_{2}\right\rangle_{\infty}$ is similar, using $i_{2 *} i_{2}^{!} j_{1 *} j_{1}^{!} C \rightarrow j_{1 *} j_{1}^{*} C \rightarrow j_{2 *} j_{2}^{*} j_{1 *} j_{1}^{*} C \rightsquigarrow$ as a second distinguished triangle. 
We have $i_{1}^{!} i_{2 *}\left(\mathcal{I}_{2}\right) \subset \mathcal{I}_{1} \cap \mathcal{I}_{2}$, hence the canonical map $i_{1 \cap *} i_{1 \cap}^{!} i_{1}^{!} i_{2 *} \stackrel{\sim}{\rightarrow} i_{1}^{!} i_{2 *}$ is an isomorphism. Now, we have canonical isomorphisms

$$
i_{1 \cap *} i_{2 \cap}^{!} \stackrel{\sim}{\rightarrow} i_{1 \cap *} i_{2 \cap}^{!} i_{2}^{!} i_{2 *} \stackrel{\sim}{\rightarrow} i_{1 \cap *} i_{1 \cap}^{!} i_{1}^{!} i_{2 *}
$$

and we get the first commutative square. The proof of the commutativity of the second square is similar.

The last assertion is clear.

Lemma 5.9. Let $\mathcal{F}$ be a finite family of Bousfield subcategories of $\mathcal{T}$ any two of which intersect properly.

Given $\mathcal{F}^{\prime}$ a subset of $\mathcal{F}$, then $\bigcap_{{\mathcal{I} \in \mathcal{F}^{\prime}}^{\prime}} \mathcal{I}$ (resp. $\left\langle\bigcup_{\mathcal{I} \in \mathcal{F}^{\prime}} \mathcal{I}\right\rangle_{\infty}$ ) is a Bousfield subcategory of $\mathcal{T}$ that intersects properly any subcategory in $\mathcal{F}$.

Given $\mathcal{I}, \mathcal{I}_{1}, \mathcal{I}_{2} \in \mathcal{F}$, then $\mathcal{I}_{1} /\left(\mathcal{I} \cap \mathcal{I}_{1}\right)$ and $\mathcal{I}_{2} /\left(\mathcal{I} \cap \mathcal{I}_{2}\right)$ are Bousfield subcategories of $\mathcal{T} / \mathcal{I}$ that intersect properly.

Proof. By induction, it is enough to prove the first assertion when $\mathcal{F}^{\prime}$ has two elements, $\mathcal{F}^{\prime}=$ $\left\{\mathcal{I}_{2}, \mathcal{I}_{3}\right\}$ and the result is then given by Lemma 5.8 .

Let $M \in \mathcal{I}_{1}, N \in \mathcal{I}_{2}, L \in \mathcal{T}$ and $f: L \rightarrow M$ and $g: L \rightarrow N$ such that $f$ becomes an isomorphism in $\mathcal{T} / \mathcal{I}$. Then, the cone of $f$ is in $\mathcal{I}$, so $L \in\left\langle\mathcal{I}_{1} \cup \mathcal{I}\right\rangle_{\infty}$. The first part of the Lemma shows that $\left\langle\mathcal{I}_{1} \cup \mathcal{I}\right\rangle_{\infty}$ and $\mathcal{I}_{2}$ intersect properly. It follows that $g$ factors through an object of $\left\langle\mathcal{I}_{1} \cup \mathcal{I}\right\rangle_{\infty} \cap \mathcal{I}_{2}$. Consequently, the image of $g$ in $\mathcal{T} / \mathcal{I}$ factors through an object of $\left(\mathcal{I}_{1} /\left(\mathcal{I} \cap \mathcal{I}_{1}\right)\right) \cap\left(\mathcal{I}_{2} /\left(\mathcal{I} \cap \mathcal{I}_{2}\right)\right)$. We have shown that every map in $\mathcal{T} / \mathcal{I}$ between $M$ and $N$ factors through an object of $\left(\mathcal{I}_{1} /\left(\mathcal{I} \cap \mathcal{I}_{1}\right)\right) \cap\left(\mathcal{I}_{2} /\left(\mathcal{I} \cap \mathcal{I}_{2}\right)\right)$ and we deduce the proper intersection property.

5.2.4. We have two Mayer-Vietoris triangles ("open" and "closed" cases).

Proposition 5.10. Assume $\mathcal{I}_{1}$ and $\mathcal{I}_{2}$ intersect properly.

(1) If $\mathcal{T}=\left\langle\mathcal{I}_{1}, \mathcal{I}_{2}\right\rangle_{\infty}$, then, there are isomorphisms of functors $i_{\cap}^{!} \stackrel{\sim}{\rightarrow} i_{1 \cap}^{!} i_{1}^{!}$and $i_{\cap}^{!} \stackrel{\sim}{\rightarrow} i_{2 \cap}^{!} i_{2}^{!}$ giving a distinguished triangle of functors

$$
i_{\cap *} i_{\cap}^{!} \stackrel{i_{1 *} \varepsilon_{1 \cap} i_{1}^{!}+i_{2 *} \varepsilon_{2 \cap} i_{2}^{!}}{\longrightarrow} i_{1 *} i_{1}^{!} \oplus i_{2 *} i_{2}^{!} \stackrel{\varepsilon_{1}-\varepsilon_{2}}{\longrightarrow} \operatorname{id}_{\mathcal{T}} \rightsquigarrow
$$

(2) If $\mathcal{I}_{1} \cap \mathcal{I}_{2}=0$, then there are isomorphisms of functors $j_{1 *} j_{1 \cup *} \stackrel{\sim}{\rightarrow} j_{\cup *}$ and $j_{2 *} j_{2 \cup *} \stackrel{\sim}{\rightarrow} j_{\cup *}$ giving a distinguished triangle of functors

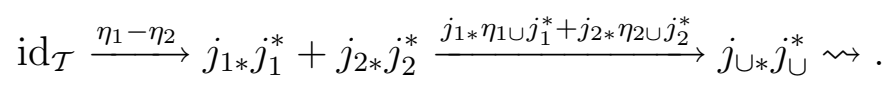

Proof. It is an easy general categorical fact that there is an isomorphism of functors $i_{\cap}^{!} \stackrel{\sim}{\rightarrow} i_{a \cap}^{!} i_{a}^{!}$ such that $\varepsilon_{\cap}=\varepsilon_{a} \circ\left(i_{a *} \varepsilon_{a \cap} i_{a}^{!}\right)$. Then, $\left(\varepsilon_{1}-\varepsilon_{2}\right) \circ\left(i_{1 *} \varepsilon_{2 \cap} i_{1}^{!}+i_{2 *} \varepsilon_{1 \cap} i_{2}^{!}\right)=0$.

Given $M \in \mathcal{I}_{2}$ an $\left(\mathcal{I}_{1} \cap \mathcal{I}_{2}\right)$-local object, then $i_{2 *} M$ is $\mathcal{I}_{1}$-local. Since the canonical functor $\mathcal{I}_{2} /\left(\mathcal{I}_{1} \cap \mathcal{I}_{2}\right) \stackrel{\sim}{\rightarrow} \mathcal{T} / \mathcal{I}_{1}$ is an equivalence, it follows that the $\mathcal{I}_{1}$-local objects of $\mathcal{T}$ are contained in $\mathcal{I}_{2}$, hence $j_{1 *}\left(\mathcal{T} / \mathcal{I}_{1}\right) \subset \mathcal{I}_{2}$. As a consequence, given $N \in \mathcal{T} / \mathcal{I}_{1}$ such that $i_{2}^{!} j_{1 *} N=0$, we have $N=0$. Consider now $C \in \mathcal{T}$ such that $i_{1}^{!} C=i_{2}^{!} C=0$. Then, $C \stackrel{\sim}{\rightarrow} j_{1 *} j_{1}^{*} C$. Since $i_{2}^{!} C=0$, it follows that $j_{1}^{*} C=0$, hence $C=0$. We deduce that in order to prove that the triangle of the Lemma is distinguished, it is sufficient to prove so after applying the functor $i_{1}^{!}$and after applying the functor $i_{2}^{!}$. 
The map $i_{1}^{!} i_{2 *} \varepsilon_{2 \cap} i_{2}^{!}: i_{1}^{!} i_{2 *} i_{2 \cap *} i_{2 \cap}^{!} i_{2}^{!} \rightarrow i_{1}^{!} i_{2 *} i_{2}^{!}$is an isomorphism since $i_{1}^{!} i_{2 *} \simeq i_{1 \cap *} i_{2 \cap}^{!}$(Lemma 5.8). As the map $i_{1}^{!} \varepsilon_{1}$ is an isomorphism, we deduce that after applying $i_{1}^{!}$, the triangle is a split distinguished triangle.

The second assertion has a similar proof.

We say that two subcategories $\mathcal{C}_{1}$ and $\mathcal{C}_{2}$ of a category $\mathcal{C}$ are orthogonal if $\operatorname{Hom}\left(C_{1}, C_{2}\right)=$ $\operatorname{Hom}\left(C_{2}, C_{1}\right)=0$ for all $C_{1} \in \mathcal{C}_{1}$ and $C_{2} \in \mathcal{C}_{2}$. Note that this is equivalent to requiring that $\mathcal{I}_{1} \cap \mathcal{I}_{2}=0$ and $\mathcal{I}_{1}$ and $\mathcal{I}_{2}$ intersect properly.

\subsection{Coverings.}

5.3.1. The following proposition shows that compactness is a local property, in a suitable sense :

Proposition 5.11. Let $\mathcal{I}_{1}$ and $\mathcal{I}_{2}$ be two orthogonal Bousfield subcategories of $\mathcal{T}$. Let $C \in \mathcal{T}$. If $j_{1}^{*} C, j_{2}^{*} C$ and $j_{\cup}^{*} C$ are compact, then $C$ is compact.

Proof. Let $\mathcal{F}$ be a set of objects of $\mathcal{T}$ whose direct sum exists. Let $a \in\{1,2, \cup\}$. We have canonical isomorphisms

$$
\begin{aligned}
\bigoplus_{D} \operatorname{Hom}\left(C, j_{a *} j_{a}^{*} D\right) & \stackrel{\sim}{\rightarrow} \bigoplus_{D} \operatorname{Hom}\left(j_{a}^{*} C, j_{a}^{*} D\right) \stackrel{\sim}{\rightarrow} \operatorname{Hom}\left(j_{a}^{*} C, \bigoplus_{D} j_{a}^{*} D\right) \stackrel{\sim}{\rightarrow} \operatorname{Hom}\left(j_{a}^{*} C, j_{a}^{*} \bigoplus_{D} D\right) \\
& \stackrel{\sim}{\rightarrow} \operatorname{Hom}\left(C, j_{a *} j_{a}^{*} \bigoplus_{D} D\right) .
\end{aligned}
$$

We have a commutative diagram

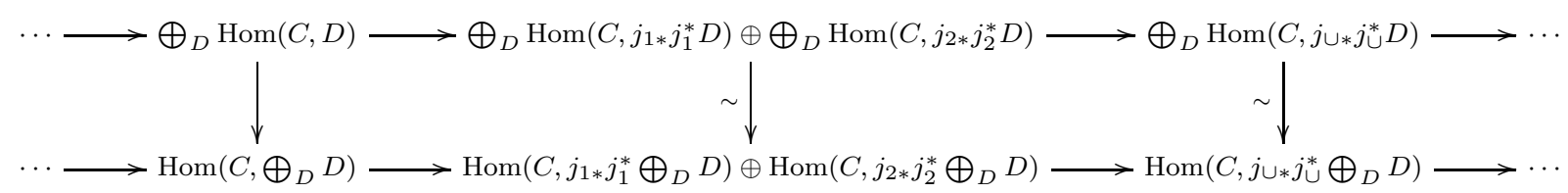

where the exact horizontal rows come from the Mayer-Vietoris triangles (Proposition $5.10(2)$ ). It follows that the canonical map $\bigoplus_{D} \operatorname{Hom}(C, D) \stackrel{\sim}{\rightarrow} \operatorname{Hom}\left(C, \bigoplus_{D} D\right)$ is an isomorphism.

Combining Theorem 5.3 and Proposition 5.11, we get

Corollary 5.12. Let $\mathcal{T}$ be a compactly generated cocomplete triangulated category and let $\mathcal{I}_{1}$ and $\mathcal{I}_{2}$ be two orthogonal Bousfield subcategories of $\mathcal{T}$. Assume $\mathcal{I}_{a}$ is compactly generated in $\mathcal{T}$ for $a \in\{1,2\}$.

Let $C \in \mathcal{T}$. Then, $C$ is compact if and only if $j_{1}^{*} C$ and $j_{2}^{*} C$ are compact.

Proof. The only new part is that the compactness of $j_{\cup}^{*} C$ follows from that of $j_{1}^{*} C$. Since compact objects of $\mathcal{T}$ remain compact in $\mathcal{T} / \mathcal{I}_{1}$ (Theorem 5.3), it follows that $\mathcal{I}_{2}$ is compactly generated in $\mathcal{T} / \mathcal{I}_{1}$. So, if $j_{1}^{*} C$ is compact, then $j_{\cup}^{*} C$ is compact (Theorem 5.3 again).

5.3.2. We have now a converse to the localization Theorem 5.3:

Proposition 5.13. Let $\mathcal{T}$ be a triangulated category and $\mathcal{I}$ be a Bousfield subcategory of $\mathcal{T}$.

Let $\mathcal{E}$ be a set of objects of $\mathcal{I} \cap \mathcal{T}^{c}$ generating $\mathcal{I}$ and $\mathcal{E}^{\prime}$ be a set of objects of $\mathcal{T}^{c}$ which generates $\mathcal{T} / \mathcal{I}$. Then $\mathcal{T}$ is generated by the set $\mathcal{E} \cup \mathcal{E}^{\prime}$. 
Proof. Let $C \in \mathcal{T}$ such that $\operatorname{Hom}(D[n], C)=0$ for all $D \in \mathcal{E}$ and $n \in \mathbf{Z}$. Then, using the distinguished triangle (11), we get $\operatorname{Hom}\left(D[n], i_{*} i ! C\right)=0$, hence $i^{!} C=0$. If follows that $C$ is $\mathcal{I}$-local.

Assume now in addition $\operatorname{Hom}\left(D^{\prime}[n], C\right)=0$ for all $D^{\prime} \in \mathcal{E}^{\prime}$ and $n \in \mathbf{Z}$. We have $C \stackrel{\sim}{\rightarrow} j_{*} j^{*} C$, hence $\operatorname{Hom}\left(j^{*} D^{\prime}[n], j^{*} C\right) \stackrel{\sim}{\rightarrow} \operatorname{Hom}\left(D^{\prime}[n], j_{*} j^{*} C\right)=0$. So, $j^{*} C=0$ and finally $C=0$.

Proposition 5.14. Let $\mathcal{T}$ be a cocomplete triangulated category and $\mathcal{I}_{1}, \mathcal{I}_{2}$ be two orthogonal Bousfield subcategories. Assume

- $\mathcal{T} / \mathcal{I}_{a}$ is compactly generated and

- $\mathcal{I}_{b}$ is compactly generated in $\mathcal{T} / \mathcal{I}_{a}$

for $\{a, b\}=\{1,2\}$.

Then, $\mathcal{T}$ is compactly generated.

More precisely, let $\mathcal{E}$ be a generating set of objects of $\mathcal{I}_{2}$ which are compact in $\mathcal{T} / \mathcal{I}_{1}$ and let $\mathcal{E}^{\prime}$ be a set of objects of $\left(\mathcal{T} / \mathcal{I}_{2}\right)^{c}$ generating $\mathcal{T} / \mathcal{I}_{2}$. Then,

- $\mathcal{E} \subset \mathcal{T}^{c}$

- given $M \in \mathcal{E}^{\prime}$, there is $\tilde{M} \in \mathcal{T}^{c}$ such that $j_{2}^{*} \tilde{M} \simeq M \oplus M[1]$

- $\mathcal{E} \cup\{\tilde{M}\}_{M \in \mathcal{E}^{\prime}}$ generates $\mathcal{T}$.

Let $\mathcal{J}$ be a Bousfield subcategory of $\mathcal{T}$ intersecting properly $\mathcal{I}_{1}$ and $\mathcal{I}_{2}$. Assume

- $\mathcal{J} /\left(\mathcal{I}_{a} \cap \mathcal{J}\right)$ is compactly generated in $\mathcal{T} / \mathcal{I}_{a}$ and

- $\mathcal{I}_{b} \cap \mathcal{J}$ is compactly generated in $\mathcal{T} / \mathcal{I}_{a}$

for $\{a, b\}=\{1,2\}$.

Then, $\mathcal{J}$ is compactly generated in $\mathcal{T}$.

Proof. Since $\mathcal{T}$ is cocomplete and $\mathcal{I}_{a}$ is Bousfield, it follows that $\mathcal{T} / \mathcal{I}_{a}$ is cocomplete.

Let $\mathcal{E}$ be a generating set of objects of $\mathcal{I}_{2}$ which are compact in $\mathcal{T} / \mathcal{I}_{1}$. Given $C \in \mathcal{E}$, we have $j_{2}^{*} C=j_{\cup}^{*} C=0$ and $j_{1}^{*} C$ is compact. It follows from Proposition 5.11 that $C$ is a compact object of $\mathcal{T}$. In particular $\mathcal{I}_{2}$ is compactly generated.

Let $\mathcal{E}^{\prime}$ be a set of compact objects generating $\mathcal{T} / \mathcal{I}_{2}$. Let $M \in \mathcal{E}^{\prime}$ and $D_{2}=M \oplus M[1]$. By Theorem 5.3, $D_{\cup}=j_{2 \cup}^{*} D_{2}$ is compact and there is $D_{1} \in\left(\mathcal{T} / \mathcal{I}_{1}\right)^{c}$ with an isomorphism $j_{1 \cup}^{*} D_{1} \stackrel{\sim}{\rightarrow} D_{\cup}$. Let now $\tilde{M}$ be the cocone of the sum of canonical maps $j_{2 *} D_{2} \oplus j_{1 *} D_{1} \rightarrow j_{\cup *} D_{\cup}$. We have $j_{a}^{*} \tilde{M} \simeq D_{a}$ for $a \in\{1,2, \cup\}$. It follows from Proposition 5.11 that $\tilde{M}$ is compact. Let $\mathcal{E}_{2}^{\prime}=\{\tilde{M}\}_{\tilde{M} \in \mathcal{E}^{\prime}}$. Now, Proposition 5.13 shows that $\mathcal{E} \cup \mathcal{E}_{2}^{\prime}$ generates $\mathcal{T}$.

For the case of $\mathcal{J}$, we apply the first part of the Proposition to the cocomplete triangulated category $\mathcal{J}$ with its orthogonal Bousfield categories $\mathcal{I}_{1} \cap \mathcal{J}$ and $\mathcal{I}_{2} \cap \mathcal{J}$. We obtain a generating set $\mathcal{E}_{\mathcal{J}}$ of objects of $\mathcal{J}$ with the property that their images in $\mathcal{J} /\left(\mathcal{I}_{1} \cap \mathcal{J}\right)$ and $\mathcal{J} /\left(\mathcal{I}_{2} \cap \mathcal{J}\right)$ are compact. These objects are thus compact in $\mathcal{T} / \mathcal{I}_{1}$ and $\mathcal{T} / \mathcal{I}_{2}$ by Theorem 5.3. Since $\mathcal{I}_{1}$ and $\mathcal{I}_{2}$ are compactly generated in $\mathcal{T}$, it follows from Corollary 5.12 that $\mathcal{E}_{\mathcal{J}} \subset \mathcal{T}^{c}$.

5.3.3. A cocovering of $\mathcal{T}$ is a finite set $\mathcal{F}$ of Bousfield subcategories of $\mathcal{T}$ any two of which intersect properly and such that $\cap_{\mathcal{I} \in \mathcal{F}} \mathcal{I}=0$.

The following result gives a construction of a compact generating set from (relative) compact generating sets for the quotients $\mathcal{T} / \mathcal{I}$.

Theorem 5.15. Let $\mathcal{F}$ be a cocovering of $\mathcal{T}$.

- Let $C$ be an object of $\mathcal{T}$ which is compact in $\mathcal{T} /\left\langle\bigcup_{\mathcal{I} \in \mathcal{F}^{\prime}} \mathcal{I}\right\rangle$ for all non empty $\mathcal{F}^{\prime} \subset \mathcal{F}$. Then, $C$ is compact in $\mathcal{T}$. 
Assume from now on that for all $\mathcal{I} \in \mathcal{F}$ and $\mathcal{F}^{\prime} \subset \mathcal{F}-\{\mathcal{I}\}$, then $\bigcap_{\mathcal{I}^{\prime} \in \mathcal{F}^{\prime}} \mathcal{I}^{\prime} / \bigcap_{\mathcal{I}^{\prime} \in \mathcal{F}^{\prime} \cup\{\mathcal{I}\}} \mathcal{I}^{\prime}$ is compactly generated in $\mathcal{T} / \mathcal{I}$.

- Then, $\mathcal{T}$ is compactly generated and an object of $\mathcal{T}$ is compact if and only if it is compact in $\mathcal{T} / \mathcal{I}$ for all $\mathcal{I} \in \mathcal{F}$.

- Let $\mathcal{J}$ be a Bousfield subcategory of $\mathcal{T}$ intersecting properly every element of $\mathcal{F}$ and such that for all $\mathcal{I} \in \mathcal{F}$ and $\mathcal{F}^{\prime} \subset \mathcal{F}-\{\mathcal{I}\}$, then $\mathcal{J} \cap \bigcap_{\mathcal{I}^{\prime} \in \mathcal{F}^{\prime}} \mathcal{I}^{\prime} / \mathcal{J} \cap \bigcap_{\mathcal{I}^{\prime} \in \mathcal{F}^{\prime} \cup\{\mathcal{I}\}} \mathcal{I}^{\prime}$ is compactly generated in $\mathcal{T} / \mathcal{I}$. Then, $\mathcal{J}$ is compactly generated in $\mathcal{T}$.

Proof. We prove each assertion of the Theorem by induction on the cardinality of $\mathcal{F}$.

Let $\mathcal{I}_{1} \in \mathcal{F}$. We put $\mathcal{I}_{2}=\bigcap_{\mathcal{I} \in \mathcal{F}-\left\{\mathcal{I}_{1}\right\}} \mathcal{I}$ and $\overline{\mathcal{I}}=\mathcal{I} / \mathcal{I}_{2}$. Given $\mathcal{I} \in \mathcal{F}$, we put $\overline{\mathcal{I}}=\mathcal{I} /\left(\mathcal{I} \cap \mathcal{I}_{2}\right)$, viewed as a full subcategory of $\overline{\mathcal{T}}$. Let $\overline{\mathcal{F}}=\{\overline{\mathcal{I}}\}_{\mathcal{I} \in \mathcal{F}-\left\{\mathcal{I}_{1}\right\}}$. We have canonical equivalences $\mathcal{T} / \mathcal{I} \stackrel{\sim}{\rightarrow} \overline{\mathcal{T}} / \overline{\mathcal{I}}$ and $\mathcal{T} /\left(\mathcal{I} \cap \mathcal{I}^{\prime}\right) \stackrel{\sim}{\rightarrow} \overline{\mathcal{T}} /\left(\overline{\mathcal{I}} \cap \overline{\mathcal{I}}^{\prime}\right)$. This shows that $\overline{\mathcal{F}}$ is a cocovering of $\overline{\mathcal{T}}$. Let $\tilde{\mathcal{T}}=\mathcal{T} /\left\langle\mathcal{I}_{1} \cup \mathcal{I}_{2}\right\rangle_{\infty}$. Given $\mathcal{I} \in \mathcal{F}-\left\{\mathcal{I}_{1}\right\}$, let $\tilde{\mathcal{I}}=\mathcal{I} /\left\langle\mathcal{I}_{2} \cup\left(\mathcal{I} \cap \mathcal{I}_{1}\right)\right\rangle_{\infty}$. Let $\tilde{\mathcal{F}}=\{\tilde{\mathcal{I}}\}_{\mathcal{I} \in \mathcal{F}-\left\{\mathcal{I}_{1}\right\}}$. This is a cocovering of $\tilde{\mathcal{T}}$.

Let $\mathcal{F}^{\prime}$ be a non-empty subset of $\mathcal{F}-\left\{\mathcal{I}_{1}\right\}$. We have equivalences $\overline{\mathcal{T}} /\left\langle\bigcup_{\mathcal{I}_{\mathcal{F}} \mathcal{F}^{\prime}} \overline{\mathcal{I}}\right\rangle_{\infty} \simeq \mathcal{T} /\left\langle\bigcup_{\mathcal{I} \in \mathcal{F}^{\prime}} \mathcal{I}\right\rangle_{\infty}$ and $\tilde{\mathcal{T}} /\left\langle\bigcup_{\mathcal{I} \in \mathcal{F}^{\prime}} \tilde{\mathcal{I}}\right\rangle_{\infty} \simeq \mathcal{T} /\left\langle\bigcup_{\mathcal{I} \in \mathcal{F}^{\prime}} \mathcal{I} \cup \mathcal{I}_{1}\right\rangle_{\infty}$. Let $C \in \mathcal{T}$ such that $C$ is compact in $\mathcal{T} /\left\langle\bigcup_{\mathcal{I} \in \mathcal{F}^{\prime}} \mathcal{I}\right\rangle_{\infty}$ for all non empty $\mathcal{F}^{\prime} \subset \mathcal{F}$. By induction, $C$ is compact in $\overline{\mathcal{T}}$ and in $\tilde{\mathcal{T}}$. Since it is also compact in $\mathcal{T} / \mathcal{I}_{1}$, it follows from Proposition 5.11 that $C$ is compact.

Given $\mathcal{F}^{\prime} \subset \mathcal{F}-\left\{\mathcal{I}_{1}\right\}$ and $\mathcal{I} \in \mathcal{F}-\left(\mathcal{F}^{\prime} \cup\left\{\mathcal{I}_{1}\right\}\right)$, then we have a canonical equivalence $\bigcap_{\mathcal{I}^{\prime} \in \mathcal{F}^{\prime}} \mathcal{I}^{\prime} / \bigcap_{\mathcal{I}^{\prime} \in \mathcal{F}^{\prime} \cup\{\mathcal{I}\}} \mathcal{I}^{\prime} \stackrel{\sim}{\rightarrow} \bigcap_{\mathcal{I}^{\prime} \in \mathcal{F}^{\prime}} \overline{\mathcal{I}}^{\prime} / \bigcap_{\mathcal{I}^{\prime} \in \mathcal{F}^{\prime} \cup\{\mathcal{I}\}} \overline{\mathcal{I}}^{\prime}$. This shows that $\bigcap_{\mathcal{I}^{\prime} \in \mathcal{F}^{\prime}} \mathcal{I}^{\prime} / \bigcap_{\mathcal{I}^{\prime} \in \mathcal{F}^{\prime} \cup\{\mathcal{I}\}} \mathcal{I}^{\prime}$ is compactly generated in $\overline{\mathcal{T}} / \overline{\mathcal{I}}$. By induction, we deduce that $\overline{\mathcal{T}}$ is compactly generated.

The induction hypothesis shows that $\mathcal{I}_{1}$ is compactly generated in $\overline{\mathcal{T}}$. Now, by assumption, $\mathcal{T} / \mathcal{I}_{1}$ is compactly generated and $\mathcal{I}_{2}$ is compactly generated in $\mathcal{T} / \mathcal{I}_{1}$. So, Proposition 5.14 shows that $\mathcal{T}$ is compactly generated.

Consider now $\overline{\mathcal{J}}=\mathcal{J} /\left(\mathcal{J} \cap \mathcal{I}_{2}\right)$. Then, $\overline{\mathcal{J}}$ intersects properly any $\overline{\mathcal{I}} \in \overline{\mathcal{F}}$. Also, $\overline{\mathcal{J}} /(\overline{\mathcal{J}} \cap \overline{\mathcal{I}})$ is compactly generated in $\overline{\mathcal{T}} / \overline{\mathcal{I}}$. By induction, we deduce that $\overline{\mathcal{J}}$ is compactly generated in $\overline{\mathcal{T}}$. Also, $\mathcal{J} \cap \mathcal{I}_{1}$ is compactly generated in $\overline{\mathcal{T}}$. By assumption, $\mathcal{J} /\left(\mathcal{J} \cap \mathcal{I}_{1}\right)$ and $\mathcal{J} \cap \mathcal{I}_{2}$ are compactly generated in $\mathcal{T} / \mathcal{I}_{1}$. It follows from Proposition 5.14 shows that $\mathcal{J}$ is compactly generated in $\mathcal{T}$.

Let $C \in \mathcal{T}$. By induction, the image $\bar{C}$ of $C$ in $\overline{\mathcal{T}}$ is compact if and only if $C$ is compact in $\mathcal{T} / \mathcal{I}$ for $\mathcal{I} \in \mathcal{F}-\left\{\mathcal{I}_{1}\right\}$. Now, Corollary 5.12 shows that $C$ is compact in $\mathcal{T}$ if and only if it is compact in $\overline{\mathcal{T}}$ and in $\mathcal{T} / \mathcal{I}_{1}$ and we are done.

Note that the proof of Theorem 5.15 actually provides a construction of a generating set. For example, if the generating sets in the hypotheses of the Theorem are all finite, then $\mathcal{T}$ is generated by a finite set of compact objects, hence by a single compact object (and the same holds for $\mathcal{J}$ ).

Proposition 5.16. Let $\mathcal{F}$ be a cocovering of $\mathcal{T}$. Then, $\operatorname{dim} \mathcal{T}<\sum_{\mathcal{I} \in \mathcal{F}}(1+\operatorname{dim} \mathcal{T} / \mathcal{I})$.

Proof. As in the proof of Theorem 5.15, we proceed by induction on the cardinality of $\mathcal{F}$. We take $\mathcal{I}_{1} \in \mathcal{F}$ and put $\mathcal{I}_{2}=\bigcap_{\mathcal{I} \in \mathcal{F}-\left\{\mathcal{I}_{1}\right\}} \mathcal{I}$. By induction, we have $\operatorname{dim} \mathcal{T} / \mathcal{I}_{2}<\sum_{\mathcal{I} \in \mathcal{F}-\left\{\mathcal{I}_{1}\right\}}(1+$ $\operatorname{dim} \mathcal{T} / \mathcal{I})$. On the other hand, we have an essentially surjective functor $\mathcal{T} / \mathcal{I}_{1} \rightarrow \mathcal{I}_{2}$, hence $\operatorname{dim} \mathcal{I}_{2} \leq \operatorname{dim} \mathcal{T} / \mathcal{I}_{1}$ (Lemma 3.3). 
Note that this holds as well for the other two definitions of dimension of Remark 3.10 when the functor $j_{\mathcal{I} *}$ commutes with direct sums (then, $i_{\mathcal{I}}^{!}$commutes with direct sums as well) - the corresponding result is certainly more interesting. This holds in the geometric setting of $\S 6.2 .1$.

\section{DeRived CATEgories of Algebras AND SCHEMES}

We study here the concepts of $\S$ 国 for derived categories of algebras and schemes.

\subsection{Algebras.}

6.1.1. From Theorem 4.22 (3), we deduce the following result $[\mathrm{Ke}, \S 5.3]$ :

Corollary 6.1. Let $A$ be a dg algebra. Then, $D(A)^{c}=\langle A\rangle_{\infty}$.

Proposition 6.2. Let $A$ be a dg algebra and $C \in D(A)$. Then, $C$ is cohomologically locally bounded (resp. bounded above, resp. bounded below) if and only if $H^{i}(C)=0$ for $|i| \gg 0$ (resp. for $i \gg 0$, resp. for $i \ll 0)$. In particular, if $C$ is cohomologically locally finitely generated, then $C \in D^{b}(A)$.

Proof. We have $D(A)^{c}=\langle A\rangle_{\infty}$ (Corollary 6.1). Hence, $C$ is cohomologically locally bounded (resp. bounded above, resp. bounded below) if and only if $h_{C}(A[i])=0$ for $|i| \gg 0$ (resp. for $i \ll 0$, resp. for $i \gg 0)$. Since $h_{C}(A[i]) \stackrel{\sim}{\rightarrow} H^{-i}(C)$, the result follows.

6.1.2. For $A$ an algebra, we denote by $K^{-, b}\left(A\right.$-proj) (resp. $K^{-, b}(A$-Proj)) the homotopy category of right bounded complexes of finitely generated projective $A$-modules (resp. projective $A$-modules) with bounded cohomology.

Proposition 6.3. Let $A$ be an algebra. The canonical functors induce equivalences between

- $K^{b}\left(A\right.$-proj) and $D(A)^{c}$

- $K^{-, b}(A$-proj) and the full subcategory of $D(A)$ of cohomologically locally finitely presented objects

Proof. The first assertion is an immediate consequence of Corollary 6.1.

Recall that the canonical functor $K^{-, b}(A$-Proj $) \rightarrow D^{b}(A)$ is an equivalence.

We now prove the second assertion. Let $C \in D(A)$. By Corollary 6.1 and Lemma 4.6, $C$ is cohomologically locally finitely presented if and only if conditions (a) and (b) hold for $X=A$.

Let $C$ be a right bounded complex of finitely generated projective $A$-modules with bounded cohomology. Consider $r$ such that $H^{i}(C)=0$ for $i \leq r$. The canonical map from the stupid truncation $\sigma^{\geq r} C$ to $C$ is surjective on cohomology, so $C$ satisfies (a), hence $C$ is cohomologically locally finitely generated. Now, Lemma 4.26 shows that $C$ is cohomologically locally finitely presented.

Let $C$ be a cohomologically locally finitely generated object. Then, $C$ has bounded cohomology (Proposition 6.2). Let $i$ be maximal such that $H^{i}(C) \neq 0$. Up to isomorphism, we can assume $C^{j}=0$ for $j>i$. By assumption, there is a bounded complex $D$ of finitely generated projective $A$-modules and $f: D \rightarrow C$ a morphism of complexes such that $H(f)$ is onto. In particular, we have a surjection $D^{i} \rightarrow C^{i} \rightarrow H^{i}(C)$, hence $H^{i}(C)$ is finitely generated.

Let $C$ be cohomologically locally finitely presented.

Assume first $C=M$ is a complex concentrated in degree 0 . Let $f: D^{0} \rightarrow M$ be a surjection, with $D^{0}$ finitely generated projective. Then, ker $f$ is cohomologically locally finitely presented 
(Proposition 4.28), hence is the quotient of a finitely generated projective module. By induction, it follows that $M$ has a left resolution by finitely generated projective $A$-modules.

We take now for $C$ an arbitrary cohomologically locally finitely presented object. We know that $C$ has bounded cohomology and we now prove by induction on $\sup \left\{i \mid H^{i}(C) \neq 0\right\}-$ $\min \left\{i \mid H^{i}(C) \neq 0\right\}$ that $C$ is isomorphic to an object of $K^{-, b}(A$-proj).

Let $i$ be maximal such that $H^{i}(C) \neq 0$. As proven above, there is a finitely generated projective $A$-module $P$ and a morphism of complexes $f: P[-i] \rightarrow C$ such that $H^{i}(f)$ is surjective. Let $C^{\prime}$ be the cone of $f$. By Proposition 4.28, $C^{\prime}$ is again cohomologically locally finitely presented. By induction, $C^{\prime}$ is isomorphic to an object of $K^{-, b}(A$-proj) and we are done.

Corollary 6.4. Let $A$ be a noetherian algebra. Then, the full subcategory of cohomologically locally finitely presented objects of $\mathcal{T}=D(A)$ is equivalent to $D^{b}(A$-mod $)$.

Remark 6.5. For a dg algebra, there might be no non-zero cohomologically locally bounded objects (e.g., for $k\left[x, x^{-1}\right]$ with $x$ in degree 1 and differential zero). The notion of cohomologically locally finitely presented objects is more interesting for our purposes.

\subsection{Schemes.}

6.2.1. Recall that a scheme is quasi-compact and quasi-separated if it has a finite covering $\mathcal{C}$ by open affine subschemes such that given $U, U^{\prime} \in \mathcal{C}$, then $U-\left(U \cap U^{\prime}\right)$ is a closed subscheme of $U$ defined by a finite number of equations.

Let $X$ be a quasi-compact and quasi-separated scheme. The category $D(X)$ is a cocomplete triangulated category. The perfect complexes have bounded cohomology. If $X$ is in addition separated, then the canonical functor $D(X$-qcoh $) \rightarrow D(X)$ is an equivalence BöNed, Corollary 5.5]. If $X=\operatorname{Spec} R$, then $D(X) \simeq D(R$-Mod). If $X$ is a noetherian scheme, then it is quasi-compact and quasi-separated and we denote by $D_{\operatorname{coh}}(X)$ the full subcategory of $D(X)$ of complexes with coherent cohomology sheaves.

Let $U$ be a quasi-compact open subscheme of $X$ (i.e., a finite union of affine open subschemes). We denote by $D_{X-U}(X)$ the full subcategory of $D(X)$ of complexes with cohomology supported by $X-U$. We denote by $j: U \rightarrow X$ the open immersion and $i: X-U \rightarrow X$ the closed immersion. We have an exact sequence of triangulated categories

$$
0 \rightarrow D_{X-U}(X) \stackrel{i_{*}}{\rightarrow} D(X) \stackrel{j^{*}}{\rightarrow} D(U) \rightarrow 0
$$

and adjoint pairs $\left(i_{*}, i^{!}\right)$and $\left(j^{*}, j_{*}\right)$. In particular, $D_{X-U}(X)$ is a Bousfield subcategory of $D(X)$. Furthermore, $j_{*}$ has finite cohomological dimension (i.e., there is an integer $N$ such that if $C \in D(U)$ and $H^{n}(C)=0$ for $n>0$, then $H^{n}\left(j_{*} C\right)=0$ for $\left.n \geq N\right)$. Consequently, $i^{!}$ has also finite cohomological dimension.

Given $U$ and $U^{\prime}$ two quasi-compact open subschemes of $X$, then $D_{X-U}(X)$ and $D_{X-U^{\prime}}(X)$ intersect properly and $D_{X-U}(X) \cap D_{X-U^{\prime}}(X)=D_{X-\left(U \cup U^{\prime}\right)}(X)$. If $U \cup U^{\prime}=X$, then the restriction functor $D_{X-U^{\prime}}(X) \stackrel{\sim}{\rightarrow} D_{U-U^{\prime} \cap U}(U)$ is an equivalence.

Given $\mathcal{F}$ a finite family of open subschemes of $X$, then $\left\{D_{X-U}(X)\right\}_{U \in \mathcal{F}}$ is a cocovering of $D(X)$ if and only if $\mathcal{F}$ is a covering of $X$. 
6.2.2. Let us start with the study of the affine case.

The following Proposition makes [BöNee, Proposition 6.1] more precise.

Proposition 6.6. Let $A$ be a commutative ring and $f_{1}, \ldots, f_{n} \in A$. Let $I$ be the ideal of $A$ generated by $f_{1}, \ldots, f_{n}$. Let $X=\operatorname{Spec} A$ and $Z=\operatorname{Spec} A / I$.

Let $K\left(f_{1}, \ldots, f_{n}\right)=\bigotimes_{i}\left(0 \rightarrow \mathcal{O}_{X} \stackrel{f_{i}}{\rightarrow} \mathcal{O}_{X} \rightarrow 0\right)$ (with non zero terms in degrees $\left.-n, \ldots, 0\right)$.

Then,

- Let $C \in D_{Z}(X)$ such that $H^{0}(C) \neq 0$. Then, $\operatorname{Hom}_{D(X)}\left(K\left(f_{1}, \ldots, f_{n}\right), C\right) \neq 0$. Given $\phi \in R \Gamma^{0}(C)$, there are integers $d_{1}, \ldots, d_{n}>0$ such that $\phi$ is in the image of the canonical map $\operatorname{Hom}_{D(X)}\left(K\left(f_{1}^{d_{1}}, \ldots, f_{n}^{d_{n}}\right), C\right) \rightarrow \operatorname{Hom}_{D(X)}\left(\mathcal{O}_{X}, C\right)=R \Gamma^{0}(C)$.

- $K\left(f_{1}, \ldots, f_{n}\right)$ is a compact object of $D(X)$ that is a generator for $D_{Z}(X)$.

Proof. It is clear that $K\left(f_{1}, \ldots, f_{m}\right)$ is compact and supported by $Z$. Also, the first statement of the Proposition implies the second one.

We have a distinguished triangle

$$
K\left(f_{1}, \ldots, f_{m-1}\right) \stackrel{f_{m}}{\longrightarrow} K\left(f_{1}, \ldots, f_{m-1}\right) \rightarrow K\left(f_{1}, \ldots, f_{m}\right) \rightsquigarrow
$$

giving an exact sequence

$$
\operatorname{Hom}\left(K\left(f_{1}, \ldots, f_{m}\right), C\right) \rightarrow \operatorname{Hom}\left(K\left(f_{1}, \ldots, f_{m-1}\right), C\right) \stackrel{f_{m}}{\longrightarrow} \operatorname{Hom}\left(K\left(f_{1}, \ldots, f_{m-1}\right), C\right) .
$$

We prove the first assertion by induction on $m$. Since $\operatorname{Hom}\left(K\left(f_{1}, \ldots, f_{m-1}\right), C\right)$ is supported by $Z$ and non zero, it follows that the kernel of the multiplication by $f_{m}$ is not zero, hence $\operatorname{Hom}\left(K\left(f_{1}, \ldots, f_{m}\right), C\right) \neq 0$. By induction, there exists $d_{1}, \ldots, d_{m-1}>0$ and $\phi_{m-1} \in \operatorname{Hom}\left(K\left(f_{1}^{d_{1}}, \ldots, f_{m-1}^{d_{m-1}}\right), C\right)$ with image $\phi \in R \Gamma^{0}(C)$. There is $d_{m}>0$ such that $f_{m}^{d_{m}} \phi_{m-1}=0$. Then, there is $\phi_{m} \in \operatorname{Hom}\left(K\left(f_{1}^{d_{1}}, \ldots, f_{m}^{d_{m}}\right), C\right)$ with image $\phi_{m-1}$. Now, $\phi_{m}$ has image $\phi \in R \Gamma^{0}(C)$.

Lemma 6.7. Let $X=\operatorname{Spec} A$ be an affine scheme and $Z$ a closed subscheme defined by $f_{1}=\cdots=f_{n}=0$. Let $\mathcal{K}$ be the smallest additive subcategory of $D_{Z}(X)$ containing the objects $K\left(f_{1}^{d_{1}}, \ldots, f_{n}^{d_{n}}\right)$ for $d_{1}, \ldots, d_{n}>0$.

Let $a \leq b$ be two integers. Let $C \in D_{\bar{Z}}^{\leq b}(X)$ with $C^{i}$ a vector bundle for $i \geq a$. Then, there is $P \in \mathcal{K}[-b] * \mathcal{K}[-b+1] * \cdots * \mathcal{K}[-a]$ and $f: P \rightarrow C$ such that $H^{i}(\operatorname{cone}(f))=0$ for $i \geq a$.

Proof. We prove the Lemma by induction on $b-a$. By assumption, $H^{b}(X)$ has finite type. It follows from Proposition 6.6 that there is $K_{1} \in \mathcal{K}$ and $f_{1}: K_{1}[-b] \rightarrow X$ such that $H^{b}\left(f_{1}\right)$ is surjective. Let $C^{\prime}=\operatorname{cone}\left(f_{1}\right)$. By induction, there is $L \in \mathcal{K}[-b+1] * \cdots * \mathcal{K}[-a]$ and $g: L \rightarrow C^{\prime}$ such that $H^{i}(\operatorname{cone}(g))=0$ for $i \geq a$. Let $P$ be the cocone of the composition $L \rightarrow C^{\prime} \rightarrow K_{1}[-b+1]$. There is $f: P \rightarrow C$ making the following diagram commutative

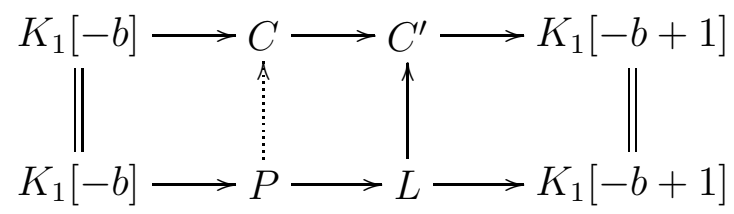

Since cone $(f) \simeq \operatorname{cone}(g)$, we are done. 
6.2.3. The following result is classical, although no published proof seems to exist (when $Z=X$, cf [Nee3, Corollary 2.3 and Proposition 2.5] for the separated case and BoVdB, Theorem 3.1.1] for the general case). The general constructions of $\$ 5.3$ reduce immediately its proof to the affine case.

Theorem 6.8. Let $X$ be a quasi-compact and quasi-separated scheme. The perfect complexes on $X$ are the compact objects of $D(X)$.

Let $Z$ be a closed subscheme of $X$ with $X-Z$ quasi-compact. Then, $D_{Z}(X)$ is generated by an object of $D_{Z}(X) \cap D(X)^{c}$.

Proof. Theorem 5.15 shows that compactness is of local nature in the following sense : an object $C \in D(X)$ is compact if and only there is a finite covering $\mathcal{C}$ of $X$ by quasi-compact open subschemes such that the restriction of $C$ to an intersection of open subschemes in $\mathcal{C}$ is compact. Perfectness is obviously also of local nature, in that sense. Since $X$ is quasi-compact and quasi-separated, we can even assume that the open subschemes in the coverings are affine. This shows that compact complexes are perfect.

Let us prove that perfect complexes are compact. The discussion above reduces the problem to proving that bounded complexes of vector bundles are compact. Corollary 6.1 shows that a bounded complex of vector bundles over an affine scheme is compact. The discussion above allows us to deduce that the same remains true for quasi-compact separated schemes, and then for quasi-compact and quasi-separated schemes.

The scheme $X$ has a finite covering $\mathcal{C}$ by affine open subschemes with $U-\left(U \cap U^{\prime}\right)$ defined by a finite number of equations for any $U, U^{\prime} \in \mathcal{C}$. Theorem 5.15 reduces then the second part of the Theorem to the case where $X$ is affine. If $Z$ is defined by the equations $f_{1}=\cdots=f_{n}=0$, then $\bigotimes_{i}\left(0 \rightarrow \mathcal{O}_{X} \stackrel{f_{i}}{\rightarrow} \mathcal{O}_{X} \rightarrow 0\right)$ is a generator of $D_{Z}(X)$ that is compact in $D(X)$ (Proposition 6.6).

Note that we deduce $D_{Z}(X)^{c}=D_{Z}(X) \cap D(X)^{c}$ (Theorem 5.3 (i)).

6.2.4. Given $C, D \in D_{Z}(X)$, we denote by $\operatorname{amp}(C)\left(\operatorname{resp} \operatorname{amp}_{D}(C)\right)$ the smallest interval of $\mathbf{Z}$ such that $H^{i}(C)=0$ for $i \notin \operatorname{amp}(C)\left(\operatorname{resp} . \operatorname{Hom}(D, C[i])=0\right.$ for $\left.i \notin \operatorname{amp}_{D}(C)\right)$. Given $I$ an interval of $\mathbf{Z}$ and $m \geq 0$, we put $I \pm m=\{i+j\}_{i \in I, j \in \mathbf{Z} \cap[-m, m]}$.

The following Proposition relates in a precise way boundedness of a complex and cohomological local boundedness (cf [BoVdB, Lemma 3.3.8] for bounded cohomology implies cohomologically locally bounded).

Proposition 6.9. Let $X$ be a quasi-compact and quasi-separated scheme and $Z$ be a closed subscheme of $X$ with $X-Z$ quasi-compact. Let $C \in D_{Z}(X)$. Then, $C$ is cohomologically locally bounded (resp. bounded above, resp. bounded below) if and only if $H^{i}(C)=0$ for $|i| \gg 0$ (resp. for $i \gg 0$, resp. for $i \ll 0)$.

More precisely, let $G \in D(X)^{c} \cap D_{Z}(X)$ be a generator for $D_{Z}(X)$. Then, there is an integer $N$ such that for any $C \in D_{Z}(X)$, then $\operatorname{amp}_{G}(C) \subset \operatorname{amp}(C) \pm N$ and $\operatorname{amp}(C) \subset \operatorname{amp}_{G}(C) \pm N$.

Proof. Let $G^{\prime} \in D_{Z}(X)^{c}$. Then, there is an integer $d$ such that $G^{\prime} \in\langle G\rangle_{d}$. As a consequence, there is an integer $m$ such that for any $C \in D_{Z}(X)$, then $\operatorname{amp}_{G^{\prime}}(C) \subset \operatorname{amp}_{G}(C) \pm m$. Note that this shows it is enough to prove the more precise statements for one $G$. 
Let us first assume that $X$ is affine. We take $G$ as in Proposition 6.6. Let $C \in D_{Z}(X)$. If $\operatorname{Hom}(G, C)=0$, then, $H^{0}(C)=0$. Conversely, If $H^{i}(C)=0$ for $-n \leq i \leq 0$, then $\operatorname{Hom}(G, C)=0$. So, the Proposition follows.

We denote by $\tilde{1}: Z \rightarrow X$ the closed immersion. Let $m$ be an integer such that for $C \in D(X)$, we have $\operatorname{amp}\left(\tilde{1}^{!} C\right) \subset \operatorname{amp}(C) \pm m$. Let $G_{0}$ be a compact generator of $D(X)$. Since $\tilde{1}_{*} G_{0}$ is compact and $G$ is a classical generator of $D(X)^{c}$, there is an integer $m^{\prime}$ such that for $C \in D(X)$, we have $\operatorname{amp}_{\tilde{1}_{*} G}(C) \subset \operatorname{amp}_{G_{0}}(C) \pm m^{\prime}$.

Let $D \in D(X)$. We have $\operatorname{Hom}\left(G, \tilde{1}^{!} D\right) \simeq \operatorname{Hom}\left(\tilde{1}_{*} G, D\right)$, so $\operatorname{amp}_{G}\left(\tilde{1}^{!} D\right)=\operatorname{amp}_{\tilde{1}_{*} G}(D) \subset$ $\operatorname{amp}_{G_{0}}(D) \pm m^{\prime}$

Let $C \in D_{Z}(X)$. Then, $\operatorname{amp}_{G}(C) \subset \operatorname{amp}_{G_{0}}\left(\tilde{1}_{*} C\right) \pm m^{\prime}$. Since $\operatorname{amp}\left(\tilde{1}_{*} C\right)=\operatorname{amp}(C)$, it follows that it is enough to prove the first inclusion of the Proposition in the case where $Z=\emptyset$. By induction, the Mayer-Vietoris triangle (Proposition 5.10 (2)) reduces the proof to the affine case, which we already considered.

Let $U_{1}, \ldots, U_{n}$ be an affine open covering of $X$. We have canonical equivalences $D_{Z-U_{r} \cap Z}(X) \stackrel{\sim}{\rightarrow}$ $D_{Z-U_{r} \cap Z}\left(\bigcup_{s \neq r} U_{s}\right)$ and $D_{U_{r} \cap Z}\left(X-\left(Z-\left(U_{r} \cap Z\right)\right)\right) \stackrel{\sim}{\rightarrow} D_{U_{r} \cap Z}\left(U_{r}\right)$. So, we have an exact sequence of triangulated categories

$$
0 \rightarrow D_{Z-U_{r} \cap Z}\left(\bigcup_{s \neq r} U_{s}\right) \stackrel{i_{*}}{\rightarrow} D_{Z}(X) \stackrel{j^{*}}{\rightarrow} D_{U_{r} \cap Z}\left(U_{r}\right) \rightarrow 0
$$

and an exact triangle of functors $i_{*} i^{!} \rightarrow \operatorname{Id}_{D_{Z}(X)} \rightarrow j_{*} j^{*} \rightsquigarrow$.

We now show the second inclusion by induction on $n$. Let $H$ be a compact generator of $D_{Z-U_{r} \cap Z}\left(\bigcup_{s \neq r} U_{s}\right)$. By induction, there is an integer $N_{1}$ such that for every $C^{\prime} \in D_{Z-U_{r} \cap Z}\left(\bigcup_{s \neq r} U_{s}\right)$, we have $\operatorname{amp}\left(C^{\prime}\right) \subset \operatorname{amp}_{H}\left(C^{\prime}\right) \pm N_{1}$. Given $C \in D_{Z}(X)$, we have $\operatorname{Hom}\left(H, i^{!} C\right) \simeq \operatorname{Hom}\left(i_{*} H, C\right)$. There is an integer $N_{2}$ such that for any $C \in D_{Z}(X)$, we have $\operatorname{amp}_{i_{*} H}(C) \subset \operatorname{amp}_{G}(C) \pm N_{2}$. So, given $C \in D_{Z}(C)$, we have $\operatorname{amp}\left(i^{!} C\right) \subset \operatorname{amp}_{G}(C) \pm N_{1} \pm N_{2}$. The proof above shows that there is $N_{3}$ such that given any $D \in D_{Z}(X)$, we have $\operatorname{amp}_{G}(D) \subset \operatorname{amp}(D) \pm N_{3}$. In particular, for any $C \in D_{Z}(X)$, we have $\operatorname{amp}_{G}\left(i_{*} i^{!} C\right) \subset \operatorname{amp}\left(i^{!} C\right) \pm N_{3} \subset \operatorname{amp}_{G}(C) \pm N_{1} \pm N_{2} \pm N_{3}$, hence $\operatorname{amp}_{G}\left(j_{*} j^{*} C\right) \subset \operatorname{amp}_{G}(C) \pm N_{1} \pm N_{2} \pm N_{3} \pm 1$.

The study of the affine case shows there is $N_{4}$ such that for any $C \in D_{Z}(X)$, then $\operatorname{amp}\left(j^{*} C\right) \subset$ $\operatorname{amp}_{j^{*} G}\left(j^{*} C\right) \pm N_{4}=\operatorname{amp}_{G}\left(j_{*} j^{*} C\right) \pm N_{4} \subset \operatorname{amp}_{G}(C) \pm N_{1} \pm N_{2} \pm N_{3} \pm N_{4} \pm 1$. There is an integer $N_{5}$ such that for any $D \in D_{U_{r} \cap Z}\left(U_{r}\right)$, we have $\operatorname{amp}\left(j_{*} D\right) \subset \operatorname{amp}(D) \pm N_{5}$. Since $\operatorname{amp}(C) \subset$ $\operatorname{amp}\left(i_{*} i^{!} C\right) \cup \operatorname{amp}\left(j_{*} j^{*} C\right)$, we deduce that $\operatorname{amp}(C) \subset \operatorname{amp}_{G}(C) \pm N_{1} \pm N_{2} \pm N_{3} \pm N_{4} \pm N_{5} \pm 1$.

6.2.5. An object $C \in D(X)$ is pseudo-coherent if for every $a \in \mathbf{Z}$ and every point $x$ of $X$, there is an open subscheme $U$ of $X$ containing $x$, a bounded complex $D$ of vector bundles on $U$ and $f \in \operatorname{Hom}_{D(U)}\left(D, C_{\mid U}\right)$ such that $H^{i}(\operatorname{cone}(f))=0$ for $i \geq a$ (cf [SGA6, §I.2] or [ThTr, $\S 2.2])$. Pseudo-coherent complexes form a thick subcategory of $D^{-}(X)$.

The following Proposition gives a substitute for global resolutions of pseudo-coherent complexes. Such resolutions exist for schemes with a family of ample line bundles (cf [SGA6, §II] or $[\overline{T h T r}$, Proposition 2.3.1]). It shows that pseudo-coherence of $C$ is a condition on the functor $\operatorname{Hom}(-, C)$ restricted to compact objects.

Proposition 6.10. Let $X$ be a quasi-compact and quasi-separated scheme and $Z$ a closed subscheme with $X-Z$ quasi-compact. Let $C \in D_{Z}(X)$. The following conditions are equivalent

(i) $C$ is pseudo-coherent 
(ii) given $a \in \mathbf{Z}$, there is $D \in D_{Z}(X)^{c}$ and $f: D \rightarrow C$ such that $H^{i}(\operatorname{cone}(f))=0$ for $i \geq a$

(iii) given any $G \in D_{Z}(X)^{c}$ and any $a \in \mathbf{Z}$, there is $D \in D_{Z}(X)^{c}$ and $f: D \rightarrow C$ such that $\operatorname{Hom}(G, \operatorname{cone}(f)[i])=0$ for $i \geq a$.

Proof. We prove (i) $\Rightarrow$ (ii) by induction on the minimal number of affine open subschemes in a covering of $X$. Let $X=U \cup V$ where $U$ is an open affine subscheme and $V$ is a an open subscheme that can be covered by strictly less affine open subschemes than $X$. Let $n$ be the minimal number of defining equations of $Z \cap(X-V)$ as a closed subscheme of $U$.

Let $C \in D_{Z}(X)$ be pseudo-coherent and let $a \in \mathbf{Z}$. Then, $C_{\mid V}$ is pseudo-coherent and by induction there is $D_{1} \in D_{Z \cap V}(V)^{c}$ and $f_{1}: D_{1} \rightarrow C_{\mid V}$ such that $H^{i}\left(\operatorname{cone}\left(f_{1}\right)\right)=0$ for $i \geq a-n$. Replacing $D_{1}$ by $D_{1} \oplus D_{1}[d]$ and $f_{1}$ by $\left(f_{1}, 0\right)$ for $d \gg 0$ odd, we can assume in addition that $\left[D_{1}\right]=0$. Then, Theorem 5.3 shows that $f_{1}$ lifts to $f_{1}^{\prime}: D_{1}^{\prime} \rightarrow C$ where $D_{1}^{\prime} \in D_{Z}(X)^{c}$. Let $C_{1}=\operatorname{cone}\left(f_{1}^{\prime}\right)$. Let $C_{2}=\tau^{\geq a-n} C_{1}$, an object of $D_{Z \cap(X-V)}(X)$. Lemma 6.7 shows there is $D_{2} \in D_{Z \cap(X-V)}(U)$ a bounded complex of free $\mathcal{O}_{U}$-modules of finite type with $D_{2}^{i}=0$ for $i<a-n$ and a map $f_{2}: D_{2} \rightarrow C_{2 \mid U}$ such that $H^{i}\left(\operatorname{cone}\left(f_{2}\right)\right)=0$ for $i \geq a$. Via the equivalence $D_{Z \cap(X-V)}(X) \stackrel{\sim}{\rightarrow} D_{Z \cap(X-V)}(U)$, this map corresponds to $f_{2}^{\prime}: D_{2}^{\prime} \rightarrow C_{2}$ with $D_{2}^{\prime} \in D_{Z \cap(X-V)}(X)^{c}$. We have

$$
\operatorname{Hom}\left(D_{2}^{\prime},\left(\tau^{<a-n} C_{1}\right)[1]\right) \simeq \operatorname{Hom}\left(D_{2},\left(\tau^{<a-n} C_{1}\right)[1]_{\mid U}\right)=0
$$

hence there is $f_{3}: D_{2}^{\prime} \rightarrow C_{1}$ lifting $f_{2}^{\prime}$. Let $C_{3}$ be its cone. We have a distinguished triangle $\tau^{<a-n} C_{1} \rightarrow C_{3} \rightarrow \operatorname{cone}\left(f_{2}^{\prime}\right) \rightsquigarrow$, hence $H^{i}\left(C_{3}\right)=0$ for $i \geq a$. Let $D$ be the cocone of the composition $C \rightarrow C_{1} \rightarrow C_{3}$. The octahedral axiom shows that $D \in D_{Z}(X)^{c}$ and we are done.

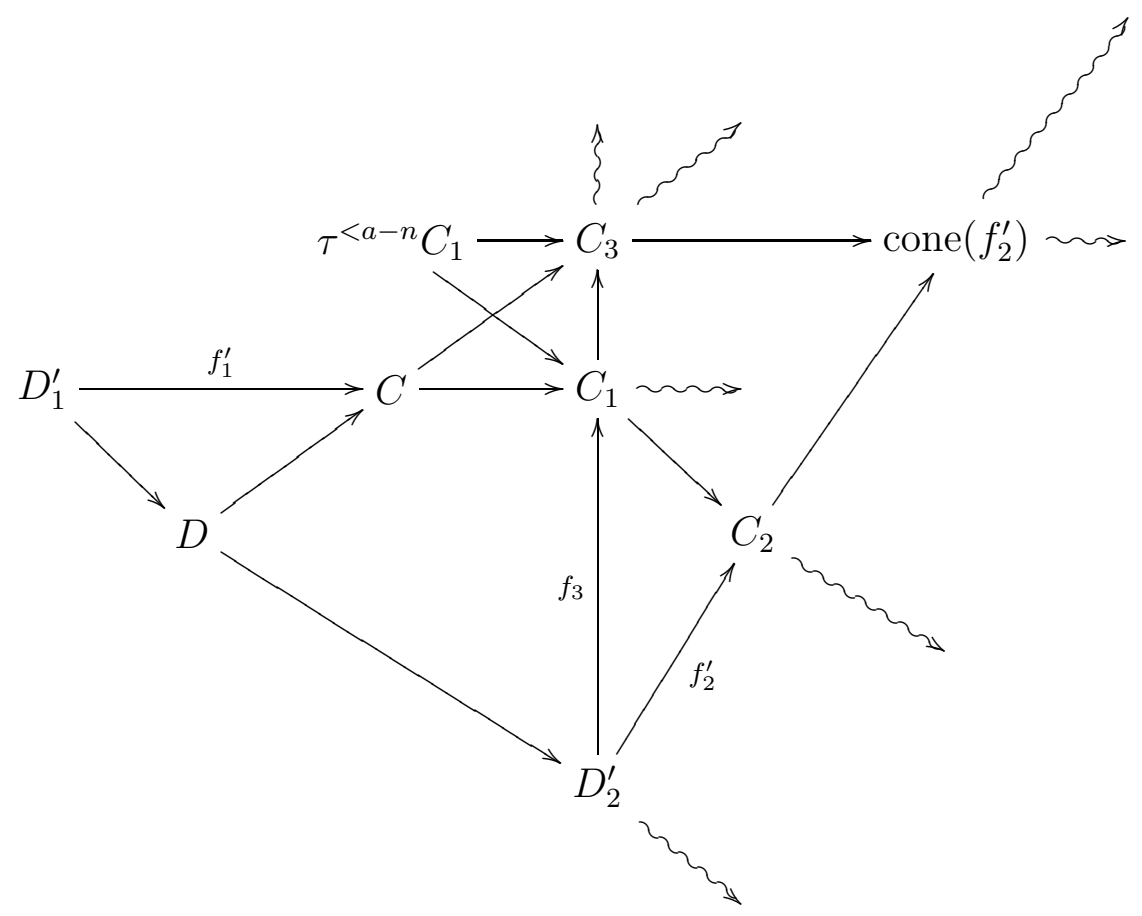

Since compact objects of $D_{Z}(X)$ are isomorphic, on affine open subschemes, to bounded complexes of vector bundles, we have (ii) $\Rightarrow(\mathrm{i})$. The equivalence between (ii) and (iii) is given by Proposition 6.9. 
We say that a noetherian scheme $X$ satisfies (*) if given $G$ a compact generator of $D(X)$ and given any $M \in X$-qcoh, there is $C \in\langle\tilde{G}\rangle_{\infty}$ and $f: C \rightarrow M$ such that $H^{0}(f)$ is surjective.

Note that if condition $(*)$ holds for one $G$, then it holds for all compact generators (cf Theorem $4.22(3))$.

Lemma 6.11. Let $X$ be an affine scheme or a quasi-projective scheme over a field. Then, $X$ satisfies $(*)$.

Proof. The affine case is clear for $G=\mathcal{O}_{X}$. The other case is solved by Lemma 7.31 below.

Proposition 6.12. Let $X$ be a noetherian scheme satisfying $(*)$. Then, the full subcategory of cohomologically locally finitely presented objects of $D(X)$ is equivalent to $D_{\text {coh }}^{b}(X)$.

Proof. Let $G$ be a compact generator for $D(X)$.

Let $M \in D_{\text {coh }}^{b}(X)$. Then, $M$ is cohomologically locally bounded (Proposition 6.9). Take $a \in \mathbf{Z}$ such that $\operatorname{Hom}(G, M[i])=0$ for $i<a$. Consider $N$ as in Proposition 6.9. By Proposition 6.10, there is $C \in D(X)^{c}$ and $f: C \rightarrow M$ such that $H^{i}$ (cone $\left.(f)\right)=0$ for $i \geq a-N$. Then, $\operatorname{Hom}(G[i], f)$ is surjective for all $i$. It follows that $M$ is cohomologically locally finitely generated. So, every object of $D_{\text {coh }}^{b}(X)$ is cohomologically locally finitely presented (Lemma 4.26).

Let $C$ be a cohomologically locally finitely presented object. Thanks to Proposition 6.9, we know that $C$ has bounded cohomology. Assume $C \notin D_{\text {coh }}^{b}(X)$ and take $i$ minimal such that $H^{i}(C)$ is not coherent. Since $\tau^{<i} C \in D_{\text {coh }}^{b}(X)$, it follows from the first part of the Proposition that $\tau^{<i} C$ is cohomologically locally finitely presented, hence $D=\tau^{\geq i} C$ is cohomologically locally finitely presented as well (Proposition 4.28). Condition (*) shows that there is $E \in\langle\tilde{G}\rangle_{\infty}$ and $f: E \rightarrow D$ such that $H^{i}(f)$ is surjective. Lemma 4.27 shows that $f$ factors through a compact object $F$. Now, $H^{i}(F)$ is coherent, hence $H^{i}(D)$ is coherent as well, a contradiction.

Remark 6.13. Let $X$ be a quasi-compact quasi-separated scheme. Let us show that given $M \in X$-qcoh of finite type, there is $C \in D(X)^{c}$ and $f: C \rightarrow M$ such that $H^{0}(f)$ is surjective.

Let $\mathcal{F}$ be a finite covering of $X$ by affine open subschemes. Given $U \in \mathcal{F}$, there is a complex $C_{U} \in D(U)^{c}$ with $\left[C_{U}\right]=0$ and a map $f_{U}: C_{U} \rightarrow \mathcal{F}_{\mid U}$ such that $H^{0}(f)$ is onto. By Theorem 5.3, there is $C(U) \in D(X)^{c}, \phi_{U}: C(U)_{\mid U} \stackrel{\sim}{\rightarrow} C_{U}$ and $f(U): C(U) \rightarrow \mathcal{F}$ such that $f(U)_{\mid U}=f_{U} \phi_{U}$. Let $C=\bigoplus_{U \in \mathcal{F}} C(U)$ and $f=\sum f(U)$. Then, $C \in D(X)^{c}$ and $f$ is surjective.

Remark 6.14. Let $k$ be a field and $X$ a projective scheme over $k$. Let $A$ be a dg algebra such that $D(A) \simeq D(X)$. Then, $H^{*}(A)$ is finite dimensional. Given $C \in D(A)$ with $H^{*}(C)$ finite dimensional and given $a \in \mathbf{Z}$, there is $D \in D(A)^{c}$ and $f: D \rightarrow C$ such that $H^{i}(\operatorname{cone}(f))=0$ for $i>a$. This is a very strong condition on a dg algebra. For example, the $\operatorname{dg}$ algebra $k[x] / x^{2}$ with $x$ in degree 1 and differential zero doesn't satisfy this condition.

\subsection{Compact objects in bounded derived categories.}

Proposition 6.15. Let $\mathcal{A}$ be an abelian category with exact filtered colimits and a set $\mathcal{G}$ of generators (i.e., a Grothendieck category). Assume that for any $G \in \mathcal{G}$, the subobjects of $G$ are compact.

Then, $\left(D^{b}(\mathcal{A})\right)^{c}=\left\langle\mathcal{A}^{c}\right\rangle_{\infty}$. 
Proof. An object $I$ of $\mathcal{A}$ is injective if and only if for any $G \in \mathcal{G}$ and any subobject $G^{\prime}$ of $G$, the canonical map $\operatorname{Hom}_{\mathcal{A}}(G, I) \rightarrow \operatorname{Hom}_{\mathcal{A}}\left(G^{\prime}, I\right)$ is surjective [Ste, Proposition V.2.9]. Note that $G^{\prime}$ is compact. It follows that a direct sum of injectives is injective.

Let $M \in \mathcal{A}^{c}$. Let $\mathcal{F}$ be a family of objects of $D^{b}(\mathcal{A})$. Then, $\bigoplus_{F \in \mathcal{F}} F$ exists in $D^{b}(\mathcal{A})$ if and only if the direct sum, computed in $D(\mathcal{A})$, has bounded cohomology, i.e., if and only if, there are integers $r$ and $s$ such that for any $F \in \mathcal{F}$, we have $H^{i}(F)=0$ for $i<r$ and for $i>s$. Given $F \in \mathcal{F}$, let $I_{F}$ be a complex of injectives quasi-isomorphic to $F$ with zero terms in degrees less than $r$. Since $\bigoplus_{F} I_{F}^{j}$ is injective, we have $\operatorname{Ext}^{i}\left(M, \bigoplus_{F} I_{F}^{j}\right)=0$ for all $j$ and $i>0$. Hence,

$$
\begin{aligned}
\bigoplus_{F} \operatorname{Hom}_{D(\mathcal{A})}(M, F) & \stackrel{\sim}{\rightarrow} \bigoplus_{F} H^{0} \operatorname{Hom}_{\mathcal{A}}\left(M, I_{F}\right) \stackrel{\sim}{\rightarrow} H^{0} \bigoplus_{F} \operatorname{Hom}_{\mathcal{A}}\left(M, I_{F}\right) \stackrel{\sim}{\rightarrow} H^{0} \operatorname{Hom}_{\mathcal{A}}\left(M, \bigoplus_{F} I_{F}\right) \\
& \stackrel{\sim}{\rightarrow} \operatorname{Hom}_{D(\mathcal{A})}\left(M, \bigoplus_{F} F\right) .
\end{aligned}
$$

It follows that $M \in D^{b}(\mathcal{A})^{c}$.

Let $C \in D^{b}(\mathcal{A})^{c}$. We prove by induction on $\max \left\{i \mid H^{i} C \neq 0\right\}-\min \left\{i \mid H^{i} C \neq 0\right\}$ that $C \in\left\langle\mathcal{A}^{c}\right\rangle_{\infty}$

Take $i$ maximal such that $H^{i} C \neq 0$. Then, $\operatorname{Hom}_{D^{b}(\mathcal{A})}(C, M[-i]) \stackrel{\sim}{\rightarrow} \operatorname{Hom}_{\mathcal{A}}\left(H^{i} C, M\right)$ for any $M \in \mathcal{A}$. It follows that $H^{i} C \in \mathcal{A}^{c}$. As proven above, we deduce that $H^{i} C[-i] \in D^{b}(\mathcal{A})^{c}$, hence $\tau^{\leq i-1} C \in D^{b}(\mathcal{A})^{c}$. By induction, $\tau^{\leq i-1} C \in\left\langle\mathcal{A}^{c}\right\rangle_{\infty}$ and we are done.

Corollary 6.16. Let $A$ be a noetherian ring. Then, $D^{b}(A$-mod $) \stackrel{\sim}{\rightarrow} D^{b}(A)^{c}$.

Let $X$ be a separated noetherian scheme. Then, $D_{\mathrm{coh}}^{b}(X) \stackrel{\sim}{\rightarrow} D^{b}(X)^{c}$.

Proof. In the ring case, we take $\mathcal{G}=\{A\}$. In the geometric case, we take for $\mathcal{G}$ the set of coherent sheaves, of [ThTr, Appendix B, §3].

\section{Dimension For DERIVED CATEGORIES OF RINGS AND SCHEMES}

\subsection{Resolution of the diagonal. Let $k$ be a field.}

\subsection{1.}

Lemma 7.1. Let $A$ be a noetherian k-algebra such that $\operatorname{pdim}_{A^{\text {en }}} A<\infty$. Then, $D^{b}(A)=$

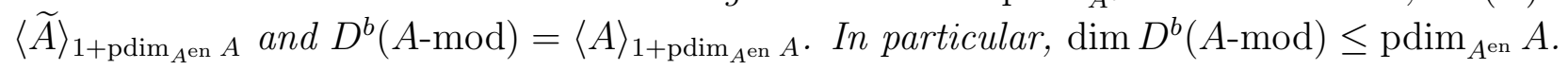

Proof. The discussion in $\oint 3.2 .2$, shows that $D^{b}(A)=\langle\widetilde{A}\rangle_{1+\operatorname{pdim}_{A^{\text {en }}} A}$. Now, we have $D^{b}(A$-mod $) \simeq$ $D^{b}(A)^{c}$ (Corollary 6.16) and the result follows from Corollary 3.13.

We say that a commutative $k$-algebra $A$ is essentially of finite type if it is the localization of a commutative $k$-algebra of finite type over $k$.

Recall the following classical result :

Lemma 7.2. Let $A$ be a finite dimensional $k$-algebra or a commutative $k$-algebra essentially of finite type. Assume that given $V$ a simple A-module, then $Z\left(\operatorname{End}_{A}(V)\right)$ is a separable extension of $k$. Then, $\operatorname{pdim}_{A^{\text {en }}} A=\operatorname{gldim} A$.

Proof. Note that under the assumptions, $A^{\mathrm{en}}$ is noetherian. In the commutative case, gldim $A=$ $\sup \left\{\operatorname{gldim} A_{\mathfrak{m}}\right\}_{\mathfrak{m}}$ and $\operatorname{pdim}_{A^{\text {en }}} A=\sup \left\{\operatorname{pdim}_{\left(A_{\mathfrak{m}}\right)^{\text {en }}} A_{\mathfrak{m}}\right\}_{\mathfrak{m}}$ where $\mathfrak{m}$ runs over the maximal ideals of $A$. It follows that it is enough to prove the commutative case of the Lemma for $A$ local. 
So, let us assume now $A$ is finite dimensional or is a commutative local $k$-algebra essentially of finite type.

Let $0 \rightarrow P^{-r} \rightarrow \cdots \rightarrow P^{0} \rightarrow A \rightarrow 0$ be a minimal projective resolution of $A$ as an $A^{\text {en }}$ module. So, there is a simple $A^{\text {en }}$-module $U$ with $\operatorname{Ext}_{A^{\text {en }}}^{r}(A, U) \neq 0$. The simple module $U$ is isomorphic to a quotient of $\operatorname{Hom}_{k}(S, T)$ for $S, T$ two simple $A$-modules. By assumption, $\operatorname{End}_{A}(S) \otimes_{k} \operatorname{End}_{A}(T)^{\circ}$ is semi-simple, hence $U$ is actually isomorphic to a direct summand of $\operatorname{Hom}_{k}(S, T)$.

Then,

$$
\operatorname{Ext}_{A}^{r}(T, S) \stackrel{\sim}{\rightarrow} \operatorname{Ext}_{A^{\text {en }}}^{r}\left(A, \operatorname{Hom}_{k}(T, S)\right) \neq 0
$$

hence, $r \leq \operatorname{gldim} A$.

Now, given $N$ an $A$-module, $0 \rightarrow P^{-r} \otimes_{A} N \rightarrow \cdots \rightarrow P^{0} \otimes_{A} N \rightarrow N \rightarrow 0$ is a projective resolution of $N$, hence $r \geq \operatorname{gldim} A$, so $r=\operatorname{gldim} A$.

Remark 7.3. Note that this Lemma doesn't hold if the residue fields of $A$ are not separable extensions of $k$. Cf the case $A=k^{\prime}$ a purely inseparable extension of $k$.

Combining Lemmas 7.1 and 7.2 , we get

Proposition 7.4. Let $A$ be a finite dimensional $k$-algebra or a commutative $k$-algebra essentially of finite type. Assume that given $V$ a simple $A$-module, then $Z\left(\operatorname{End}_{A}(V)\right)$ is a separable extension of $k$.

If $A$ has finite global dimension, then $D^{b}(A)=\langle\widetilde{A}\rangle_{1+\text { gldim } A}$ and $D^{b}(A$-mod $)=\langle A\rangle_{1+\text { gldim } A}$. In particular, $\operatorname{dim} D^{b}(A$-mod $) \leq \operatorname{gldim} A$.

Remark 7.5. The dimension of $D^{b}(A$-mod) can be strictly less than gldim $A$ (this will be the case for example for a finite dimensional $k$-algebra $A$ which is not hereditary but which is derived equivalent to a hereditary algebra). This cannot happen if $A$ is a finitely generated commutative $k$-algebra, cf Proposition 7.17 below.

7.1.2. Following $\$ 3.2 .2$, we have the following result (cf [BoVaB, §3.4]).

Proposition 7.6. Let $X$ be a separated noetherian scheme over $k$. Assume there is a vector bundle $\mathcal{L}$ on $X$ and a resolution of the structure sheaf $\mathcal{O}_{\Delta}$ of the diagonal in $X \times X$

$$
0 \rightarrow \mathcal{F}^{-r} \rightarrow \cdots \rightarrow \mathcal{F}^{0} \rightarrow \mathcal{O}_{\Delta} \rightarrow 0
$$

with $\mathcal{F}^{i} \in \operatorname{add}(\mathcal{L} \otimes \mathcal{L})$.

Then, $D^{b}(X$-qcoh $)=\langle\widetilde{\mathcal{L}}\rangle_{1+r}$ and $D^{b}(X-\mathrm{coh})=\langle\mathcal{L}\rangle_{1+r}$.

Proof. Let $p_{1}, p_{2}: X \times X \rightarrow X$ be the first and second projections. For $C \in D^{b}(X$-qcoh), we have $C \simeq R p_{1 *}\left(\mathcal{O}_{\Delta} \otimes^{\mathbf{L}} p_{2}^{*} C\right)$. It follows that $C \in\left\langle\mathcal{L} \otimes_{k} R \Gamma(\mathcal{L} \otimes C)\right\rangle_{1+r}$, hence $C \in\langle\widetilde{\mathcal{L}}\rangle_{1+r}$. Since $D^{b}(X \text {-qcoh })^{c}=D^{b}(X$-coh) (Corollary 6.16), the second assertion follows from Corollary 3.13 .

Note that the assumption of the Proposition forces $X$ to be smooth.

Example 7.7. Let $X=\mathbf{P}_{k}^{n}$. Let us recall results of Beilinson [Bei]. The object $G=\mathcal{O} \oplus$ $\cdots \oplus \mathcal{O}(n)$ is a classical generator for $D^{b}\left(X\right.$-coh). We have $\operatorname{Ext}^{i}(G, G)=0$ for $i \neq 0$. Let $A=\operatorname{End}(G)$. We have $D^{b}(X$-coh $) \simeq D^{b}(A$-mod $)$. We have gldim $A=n$, hence $D^{b}(A$-mod $)=$ 
$\langle A\rangle_{n+1}$ (Proposition 7.4), so $D^{b}\left(\mathbf{P}^{n}\right.$-coh) $=\langle\mathcal{O} \oplus \cdots \oplus \mathcal{O}(n)\rangle_{n+1}$. Another way to see this is to use the resolution of the diagonal $\Delta \subset X \times X$ :

$$
0 \rightarrow \mathcal{O}(-n) \otimes \Omega^{n}(n) \rightarrow \cdots \rightarrow \mathcal{O}(-1) \otimes \Omega^{1}(1) \rightarrow \mathcal{O} \otimes \mathcal{O} \rightarrow \mathcal{O}_{\Delta} \rightarrow 0 .
$$

By Proposition 7.17 below, it follows that $\operatorname{dim} D^{b}\left(\mathbf{P}^{n}-\mathrm{coh}\right)=n$.

Example 7.8. In Kap, Kapranov considers flag varieties (type $A$ ) and smooth projective quadrics. For these varieties $X$, he constructs explicit bounded resolutions of the diagonal whose terms are direct sums of $\mathcal{L} \otimes \mathcal{L}^{\prime}$, where $\mathcal{L}$ and $\mathcal{L}^{\prime}$ are vector bundles. It turns out that these resolutions have exactly $1+\operatorname{dim} X$ terms (this is the smallest possible number). By Proposition 7.17, it follows that $\operatorname{dim} D^{b}(X$-coh $)=\operatorname{dim} X$.

Starting from a smooth projective variety $X$, there is an ample line bundle whose homogeneous coordinate ring is a Koszul algebra $\left[\mathrm{Ba}\right.$, Theorem 2]. This provides a resolution of $\mathcal{O}_{\Delta}$ [Kaw, Theorem 3.2]. Now, if the kernel of the $r$-th map of the resolution is a direct sum of sheaves of the form $\mathcal{L} \otimes \mathcal{L}^{\prime}$, where $\mathcal{L}, \mathcal{L}^{\prime}$ are vector bundles, then $\operatorname{dim} D^{b}(X$-coh $) \leq r$. Note that this can work only if the class of $\mathcal{O}_{\Delta}$ is in the image of the product map $K_{0}(X) \times K_{0}(X) \rightarrow$ $K_{0}(X \times X)$. The case of flag varieties associated to reductive groups of type different from $A_{n}$ would be interesting to study.

The following is our best result providing an upper bound for smooth schemes.

Proposition 7.9. Let $X$ be a smooth quasi-projective scheme over $k$. Let $\mathcal{L}$ be an ample line bundle on $X$. Then, there is $r \geq 0$ such that $D^{b}(X$-qcoh $)=\langle\widetilde{G}\rangle_{2 \operatorname{dim} X+1}$ and $D^{b}(X-\operatorname{coh})=$ $\langle G\rangle_{2 \operatorname{dim} X+1}$ where $G=\mathcal{O} \oplus \mathcal{L}^{\otimes-1} \oplus \cdots \oplus \mathcal{L}^{\otimes-r}$. In particular, $\operatorname{dim} D^{b}(X$-coh $) \leq 2 \operatorname{dim} X$.

Proof. There is a resolution of the diagonal

$$
\cdots \rightarrow C^{-i} \stackrel{d^{-i}}{\longrightarrow} \cdots \rightarrow C^{0} \stackrel{d^{0}}{\longrightarrow} \mathcal{O}_{\Delta} \rightarrow 0
$$

where $C^{i} \in \operatorname{add}\left(\left\{\mathcal{L}^{-j} \otimes \mathcal{L}^{-j}\right\}_{j \geq 0}\right)$. Denote by $C$ the complex $\cdots \rightarrow C^{-i} \stackrel{d^{-i}}{\longrightarrow} \cdots \rightarrow C^{0} \rightarrow 0$. Let $n=\operatorname{dim} X$. Truncating, we get an exact sequence

$$
0 \rightarrow C^{-2 n-1} / \operatorname{ker} d^{-2 n} \rightarrow C^{-2 n} \rightarrow \cdots \rightarrow C^{-i} \stackrel{d^{-i}}{\longrightarrow} \cdots \rightarrow C^{0} \stackrel{d^{0}}{\longrightarrow} \mathcal{O}_{\Delta} \rightarrow 0
$$

Since $X \times X$ is smooth of dimension $2 n$, we have $\operatorname{Ext}^{2 n+1}\left(\mathcal{O}_{\Delta}, C^{-2 n-1} / \operatorname{ker} d^{-2 n}\right)=0$. So, the distinguished triangle $C^{-2 n-1} / \operatorname{ker} d^{-2 n}[2 n] \rightarrow \sigma^{\geq-2 n} C \rightarrow \mathcal{O}_{\Delta} \rightsquigarrow$ splits, i.e., $\mathcal{O}_{\Delta}$ is a direct summand of the complex $\sigma^{\geq-2 n} C$. We conclude as in the proof of Proposition 7.6 .

Remark 7.10. We actually don't know any case of a smooth variety where $\operatorname{dim} D^{b}(X$-coh $)>$ $\operatorname{dim} X$. The first case to consider would be an elliptic curve.

Remark 7.11. Let $d$ be the largest integer such that $\operatorname{Ext}_{\mathcal{O}_{X \times X}}^{d}\left(\mathcal{O}_{\Delta X}, \mathcal{F}\right) \neq 0$ for some $\mathcal{F} \in$ $(X \times X)$-coh. Then, $\operatorname{dim} X \leq d$. We don't know if the inequality can be strict.

7.1.3. For applications to finite dimensional algebras, we need to prove certain results for the derived category of differential modules. The theory of such derived categories mirrors that of the usual derived category of complexes of modules (forget the grading). We state here the constructions and results needed in this paper.

Let $A$ be a $k$-algebra. A differential $A$-module is an $\left(A \otimes_{k} k[\varepsilon] /\left(\varepsilon^{2}\right)\right)$-module. We view a differential $A$-module as a pair $(M, d)$ where $M$ is an $A$-module and $d \in \operatorname{End}_{A}(M)$ satisfying 
$d^{2}=0$ is given by the action of $\varepsilon$. The cohomology of a differential $A$-module is the $A$-module $\operatorname{ker} d / \operatorname{im} d$.

The category of differential $A$-modules has the structure of an exact category, where the exact sequences are those exact sequences of $\left(A \otimes_{k} k[\varepsilon] /\left(\varepsilon^{2}\right)\right)$-modules that split by restriction to $A$. This is a Frobenius category and its associated stable category is called the homotopy category of differential $A$-modules.

A morphism of $A$-modules is a quasi-isomorphism if the induced map on cohomology is an isomorphism. We now define the derived category of differential $A$-modules, denoted by $D \operatorname{diff}(A)$, as the localization of the homotopy category of differential $A$-modules in the class of quasi-isomorphisms. These triangulated categories have a trivial shift functor.

We have a triangulated forgetful functor $D(A) \rightarrow D \operatorname{diff}(A)$. Let $X, Y$ be two $A$-modules and $i \geq 0$. Then, the canonical map $\operatorname{Ext}_{A}^{i}(X, Y) \stackrel{\sim}{\rightarrow} \operatorname{Hom}_{D(A)}(X, Y[i]) \rightarrow \operatorname{Hom}_{D \operatorname{diff}(A)}(X, Y)$ is injective and we have an isomorphism $\prod_{n \geq 0} \operatorname{Ext}_{A}^{n}(X, Y) \stackrel{\sim}{\rightarrow} \operatorname{Hom}_{D \operatorname{diff}(A)}(X, Y)$.

\subsection{4.}

Lemma 7.12. Let $A$ be a k-algebra. Let $W$ be an $A$-module with $\operatorname{pdim} W \geq d$. Then, there are $A^{\mathrm{en}}$-modules $M_{0}=A, M_{1}, \ldots, M_{d}$ which are projective as left and as right $A$-modules, and elements $\zeta_{i} \in \operatorname{Ext}_{A^{\mathrm{en}}}^{1}\left(M_{i}, M_{i+1}\right)$ for $0 \leq i \leq d-1$ such that $\left(\zeta_{d-1} \cdots \zeta_{0}\right) \otimes_{A} \mathrm{id}_{W}$ is a non zero element of $\operatorname{Ext}_{A}^{d}\left(W, M_{d} \otimes_{A} W\right)$.

Proof. Let $\cdots \rightarrow C^{-2} \stackrel{d^{-2}}{\longrightarrow} C^{-1} \stackrel{d^{-1}}{\longrightarrow} C^{0} \stackrel{d^{0}}{\longrightarrow} A \rightarrow 0$ be a projective resolution of the $A^{\text {en }}$-module $A$. Then, $\cdots \rightarrow C^{-2} \otimes_{A} W \rightarrow C^{-1} \otimes_{A} W \rightarrow C^{0} \otimes_{A} W \rightarrow W \rightarrow 0$ is a projective resolution of $W$. Let $\Omega^{-i}$ be the kernel of $d^{i+1}$ for $i \leq-1$ and $\Omega^{0}=A$. Let $\zeta_{i} \in \operatorname{Ext}_{A^{\text {en }}}^{1}\left(\Omega^{i}, \Omega^{i+1}\right)$ given by the exact sequence $0 \rightarrow \Omega^{i+1} \rightarrow C^{-i} \stackrel{d^{-i}}{\longrightarrow} \Omega^{i} \rightarrow 0$.

Since $\operatorname{Ext}_{A}^{d}(W,-)$ is not zero, it follows that the exact sequence

$$
0 \rightarrow \Omega^{d} \otimes_{A} W \rightarrow C^{-d+1} \otimes_{A} W \rightarrow \cdots \rightarrow C^{-1} \otimes_{A} W \rightarrow C^{0} \otimes_{A} W \rightarrow W \rightarrow 0
$$

gives a non zero element $\xi \in \operatorname{Ext}_{A}^{d}\left(W, \Omega^{d} \otimes_{A} W\right)$. This element is equal to $\left(\zeta_{d-1} \cdots \zeta_{0}\right) \otimes_{A} \mathrm{id}_{W}$.

The following result is our main tool to produce lower bounds for the dimension.

Lemma 7.13. Let $A$ be a k-algebra. Let $W$ be an $A$-module with $\operatorname{pdim} W \geq d$. Let $\mathcal{T}$ be $D(A)$ or $\operatorname{Ddiff}(A)$. Then, $W \notin\langle\bar{A}\rangle_{\mathcal{T}, d}$.

Proof. Assume $W \in\langle\bar{A}\rangle_{1+r}$ for some $r \geq 0$. Let $W_{s-1} \rightarrow W_{s} \rightarrow V_{s} \rightsquigarrow$ be a family of distinguished triangles, for $1 \leq s \leq r$. We put $V_{0}=W_{0}$ and we assume $V_{s} \in\langle\bar{A}\rangle$ for $0 \leq s \leq r$ and $W_{r}=W \oplus W^{\prime}$ for some $W^{\prime}$.

We use now Lemma 7.12. The element $\zeta_{i}$ induces a natural transformation of functors $M_{i} \otimes_{A}-\rightarrow M_{i+1}[1] \otimes_{A}-$ from $\mathcal{T}$ to itself. Restricted to $\langle\bar{A}\rangle$, this transformation is zero. It follows from Lemma 4.11 that $\left(\zeta_{d-1} \cdots \zeta_{0}\right) \otimes_{A}-$ vanishes on $\langle\bar{A}\rangle_{d}$. It follows that $r \geq d$ (in case $\mathcal{T}$ is the derived category of differential $A$-modules, note that the canonical map $\operatorname{Ext}_{A}^{d}\left(W, M_{d} \otimes_{A} W\right) \rightarrow \operatorname{Hom}_{\mathcal{T}}\left(W, M_{d} \otimes_{A} W\right)$ is injective $)$.

We deduce the following crucial Proposition :

Proposition 7.14. Let $A$ be a commutative local noetherian $k$-algebra with maximal ideal $\mathfrak{m}$. Let $\mathcal{T}$ be $D(A)$ or $D \operatorname{diff}(A)$. Then, $A / \mathfrak{m} \notin\langle A\rangle_{\mathcal{T}, \operatorname{Krulldim} A}$. 
Proof. We know that $\operatorname{Krulldim} A \leq \operatorname{gldim} A=\operatorname{pdim}_{A} A / \mathfrak{m}$ (cf for example [Ma, Theorem 41]). The result follows now from Lemma 7.13.

Remark 7.15. Let $M, V \in D(A)$. If $V \in\langle\bar{M}\rangle_{D(A), i}$, then $F(V) \in\langle\overline{F(M)}\rangle_{D \operatorname{diff}(A), i}$, where $F: D(A) \rightarrow D \operatorname{diff}(A)$ is the forgetful functor.

From Lemma 7.13 and Propositions 7.4 and 7.26 , we deduce

Proposition 7.16. Let $A$ be a noetherian k-algebra of global dimension $d \in \mathbf{N} \cup\{\infty\}$. Assume $k$ is perfect. Then, $d$ is the minimal integer $i$ such that $A$-perf $=\langle A\rangle_{i+1}$.

We can now bound dimensions :

Proposition 7.17. Let $X$ be a reduced separated scheme of finite type over $k$. Then, we have $\operatorname{dim} D^{b}(X-\operatorname{coh}) \geq \operatorname{dim} X$.

Proof. Let $M \in D^{b}(X$-coh $)$ such that $D^{b}(X$-coh $)=\langle M\rangle_{r+1}$.

Pick a closed point $x$ of $X$ with local ring $\mathcal{O}_{x}$ of Krull dimension $\operatorname{dim} X$ such that $M_{x} \in\left\langle\mathcal{O}_{X}\right\rangle$ (given $F$ a coherent sheaf over $X$, there is a dense open affine $U$ such that $F_{\mid U}$ is projective. Now, a complex with projective cohomology splits). Then, $k_{x} \in\left\langle\mathcal{O}_{x}\right\rangle_{r+1}$. It follows from Proposition 7.14 that $r \geq \operatorname{Krulldim} \mathcal{O}_{x}=\operatorname{dim} X$.

From Propositions 7.4 and 7.17, we deduce

Theorem 7.18. Let $X$ be a smooth affine scheme of finite type over $k$. Then, $\operatorname{dim} D^{b}(X$-coh $)=$ $\operatorname{dim} X$.

Remark 7.19. Let $A=k[x] /\left(x^{2}\right)$ be the algebra of dual numbers. The indecomposable objects of $D^{b}\left(A\right.$-mod) are $k[i]$ and $L_{n}[i]$ for $n \geq 1$ and $i \in \mathbf{Z}$, where $L_{n}$ is the cone of a non-zero map $k \rightarrow k[n]$. It follows that $D^{b}(A$-mod $)=\langle k\rangle_{2}$, hence, $\operatorname{dim} D^{b}(A$-mod $)=1($ cf Proposition 7.38 below).

Note that the dimension of the category of perfect complexes of $A$-modules is infinite by Proposition 7.26 below. Let us prove this directly. Given $C$ a perfect complex of $A$-modules, there is an integer $r$ such that $\operatorname{Ext}_{A^{\text {en }}}^{i}(A, A)$ acts as 0 on $\langle C\rangle$ for $i \geq r$. On the other hand, given $d$ an integer, then the canonical map $\left.-\otimes_{A} \operatorname{id}_{L_{r d+1}}: \operatorname{Ext}_{A^{\text {en }}}^{1}(A, A)\right)^{r d} \rightarrow \operatorname{Hom}_{D^{b}(A)}\left(L_{r d+1}, L_{r d+1}[r d]\right)$ is not zero (note that $L_{r d+1}$ is perfect). So, $L_{r d+1} \notin\langle C\rangle_{d}$ by Lemma 4.11.

Remark 7.20. Let $k$ be a field and $A$ a finitely generated $k$-algebra. Can the dimension of $D^{b}(A$-mod) be infinite ? We will show that the dimension is finite if $A$ is finite dimensional (Proposition 7.38) or commutative and $k$ is perfect (Theorem 7.39).

\subsection{Finite global dimension.}

7.2.1. We explain here a method of dévissage for derived categories of abelian categories with finite global dimension.

Lemma 7.21. Let $\mathcal{A}$ be an abelian category and $C$ a complex of objects of $\mathcal{A}$. Assume $H^{1} C=$ $\cdots=H^{i} C=0$ for some $i \geq 0$. Let $0 \rightarrow \operatorname{ker} d^{0} \stackrel{\alpha}{\longrightarrow} L^{0} \stackrel{f^{0}}{\longrightarrow} \cdots \stackrel{f^{i}}{\longrightarrow} L^{i+1} \stackrel{\beta}{\longrightarrow} C^{i+1} / \operatorname{im} d^{i} \rightarrow 0$ be an exact sequence equivalent to $0 \rightarrow \operatorname{ker} d^{0} \rightarrow C^{0} \rightarrow \cdots \rightarrow C^{i+1} \rightarrow C^{i+1} /$ im $d^{i} \rightarrow 0$ (i.e., giving the same element in $\operatorname{Ext}^{i+2}\left(C^{i+1} / \operatorname{im} d^{i}\right.$, $\left.\left.\operatorname{ker} d^{0}\right)\right)$. Then, $C$ is quasi-isomorphic to the complex

$$
\cdots \rightarrow C^{-2} \stackrel{d^{-2}}{\longrightarrow} C^{-1} \stackrel{a}{\longrightarrow} L^{0} \stackrel{f^{0}}{\longrightarrow} \cdots \stackrel{f^{i}}{\longrightarrow} L^{i+1} \stackrel{b}{\longrightarrow} C^{i+2} \stackrel{d^{i+2}}{\longrightarrow} \cdots
$$


where $a$ is the composite $C^{-1} \stackrel{d^{-1}}{\longrightarrow} \operatorname{ker} d^{0} \stackrel{\alpha}{\longrightarrow} L^{0}$ and $b$ the composite $L^{i+1} \stackrel{\beta}{\longrightarrow} C^{i+1} /$ im $d^{i} \stackrel{d^{i+1}}{\longrightarrow}$ $C^{i+2}$.

Proof. It is enough to consider the case of an elementary equivalence between exact sequences. Let

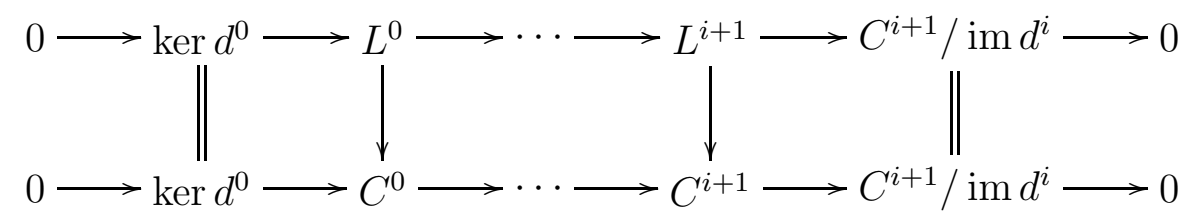

be a commutative diagram, with the rows being exact sequences. Then, there is a commutative diagram

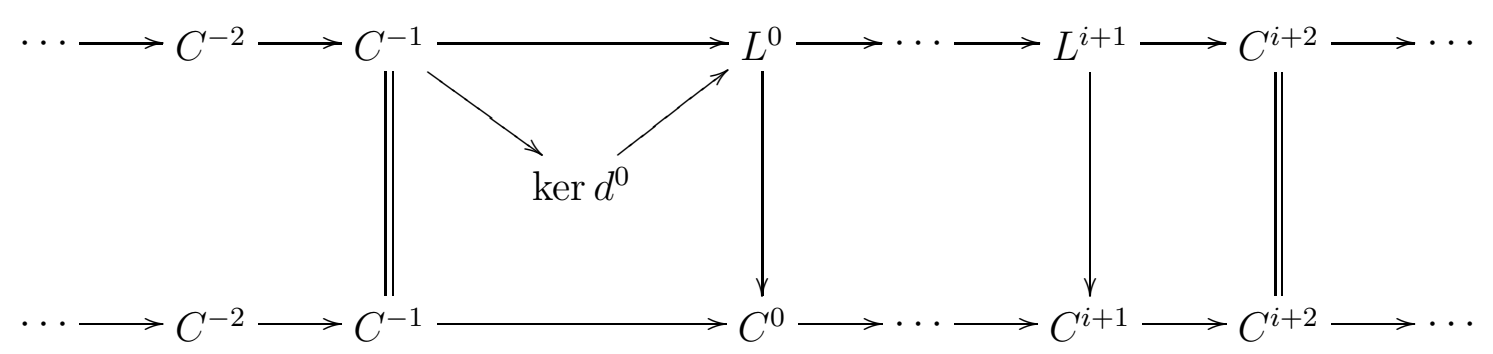

This induces a morphism of complexes from the first row to the last row of the diagram and this is a quasi-isomorphism.

Lemma 7.22. Let $\mathcal{A}$ be an abelian category with finite global dimension $\leq n$. Let $C$ be a complex of objects of $\mathcal{A}$. Assume $H^{i} C=0$ if $n \backslash i$. Then, $C$ is quasi-isomorphic to $\bigoplus_{i}\left(H^{n i} C\right)[-n i]$.

Proof. Pick $i \in \mathbf{Z}$. The sequence $0 \rightarrow \operatorname{ker} d^{n i} \rightarrow C^{n i} \rightarrow \cdots \rightarrow C^{n(i+1)} \rightarrow C^{n(i+1)} / \operatorname{im} d^{n(i+1)-1} \rightarrow$ 0 is exact. It defines an element of $\operatorname{Ext}_{\mathcal{A}}^{n+1}\left(C^{n(i+1)} / \operatorname{im} d^{n(i+1)-1}, \operatorname{ker} d^{n i}\right)$. This group is 0 by assumption, hence the exact sequence is equivalent to $0 \rightarrow \operatorname{ker} d^{n i} \rightarrow \operatorname{ker} d^{n i} \stackrel{0}{\longrightarrow} 0 \cdots 0 \stackrel{0}{\longrightarrow}$ $C^{n(i+1)} / \operatorname{im} d^{n(i+1)-1} \rightarrow C^{n(i+1)} / \operatorname{im} d^{n(i+1)-1} \rightarrow 0$. Lemma 7.21 shows that $C$ is quasi-isomorphic to a complex $D$ with $d_{D}^{n i}=\cdots=d_{D}^{n(i+1)-1}=0$. Now, there is a morphism of complexes $\left(H^{n(i+1)} C\right)[-n(i+1)] \rightarrow D$ that induces an isomorphism on $H^{n(i+1)}$. So, for every $i$, there is a map $\rho_{i}$ in $D(\mathcal{A})$ from $\left(H^{n i} C\right)[-n i]$ to $C$ that induces an isomorphism on $H^{n i}$. Let $\rho=\sum_{i} \rho_{i}$ : $\bigoplus_{i}\left(H^{n i} C\right)[-n i] \rightarrow C$. This is a quasi-isomorphism.

Proposition 7.23. Let $\mathcal{A}$ be an abelian category with finite global dimension $\leq n$ with $n \geq 1$. Let $C$ be a complex of objects of $\mathcal{A}$. Then, there is a distinguished triangle in $D(\mathcal{A})$

$$
\bigoplus_{i} D_{i} \rightarrow C \rightarrow \bigoplus_{i} E_{i} \rightsquigarrow
$$

where $D_{i}=\sigma^{\geq n i+1} \tau^{\leq n(i+1)-1} C$ is a complex with zero terms outside $[n i+1, \ldots, n(i+1)-1]$ and $E_{i}$ is a complex concentrated in degree $n i$.

Proof. Let $i \in \mathbf{Z}$. Let $f_{i}$ be the composition of the canonical maps $\tau^{\leq n(i+1)-1} C \rightarrow C$ with the canonical map $\sigma^{\geq n i+1} \tau^{\leq n(i+1)-1} C \rightarrow \tau^{\leq n(i+1)-1} C$. Then, $H^{r}\left(f_{i}\right)$ is an isomorphism for $n i+2 \leq r \leq n(i+1)-1$ and is surjective for $r=n i+1$. Let $D=\bigoplus_{i} \sigma^{\geq n i+1} \tau^{\leq n(i+1)-1} C$ and 
$f=\sum_{i} f_{i}: D \rightarrow C$. Let $E$ be the cone of $f$. We have an exact sequence

$$
\begin{aligned}
\cdots \rightarrow H^{n i-2} D & \stackrel{\sim}{\rightarrow} H^{n i-2} C \rightarrow H^{n i-2} E \rightarrow H^{n i-1} D \stackrel{\sim}{\rightarrow} H^{n i-1} C \rightarrow H^{n i-1} E \rightarrow H^{n i} D \rightarrow \\
\rightarrow H^{n i} C \rightarrow H^{n i} E & \rightarrow H^{n i+1} D \rightarrow H^{n i+1} C \rightarrow H^{n i+1} E \rightarrow H^{n i+2} D \stackrel{\sim}{\rightarrow} H^{n i+2} C \rightarrow \cdots
\end{aligned}
$$

Since $H^{n i} D=0$ for all $i$, we deduce that $H^{r} E=0$ if $n \backslash \gamma r$. The Proposition follows now from Lemma 7.22 .

Remark 7.24. Note there is a dual version to Proposition 7.23 obtained by passing to the opposite category $\mathcal{A}^{\circ}$.

\subsection{2.}

Proposition 7.25. Let $A$ be a ring with finite global dimension. Then, $D^{b}(A)=\langle\widetilde{A}\rangle_{2+2 \operatorname{gldim} A}$. If $A$ is noetherian, then $D^{b}(A$-mod $)=\langle A\rangle_{2+2 \operatorname{gldim} A}$ and $\operatorname{dim} D^{b}(A$-mod $) \leq 1+2 \operatorname{gldim} A$.

Proof. Put $n=\operatorname{gldim} A$. Let $C \in D^{b}(A)$. Up to quasi-isomorphism, we can assume $C$ is a bounded complex of projective $A$-modules. We now use Proposition 7.23. An $A$-module $M$ has a projective resolution of length $n+1$, hence $M \in\langle\widetilde{A}\rangle_{n+1}$. So, $\bigoplus_{i} E_{i} \in\langle\widetilde{A}\rangle_{n+1}$. Similarly, we have $\bigoplus_{i} D_{i} \in\langle\widetilde{A}\rangle_{n+1}$, hence $C \in\langle\widetilde{A}\rangle_{2+2 n}$.

The second part of the Lemma follows from Corollaries 6.16 and 3.13 .

The following characterization of regular algebras is due to Van den Bergh in the noetherian case. It characterizes regularity as a property of $D(A)$ as a triangulated category.

Proposition 7.26. Let $A$ be a ring. Then, the following conditions are equivalent

(i) $A$ is regular, i.e., gldim $A<\infty$

(ii) $K^{b}\left(A\right.$-Proj) $\stackrel{\sim}{\rightarrow} D^{b}(A)$

(iii) there is $G \in D(A)^{c}$ and $d \in \mathbf{N}$ such that $\left\langle\widetilde{D(A)^{c}}\right\rangle_{\infty}=\langle\tilde{G}\rangle_{d}$

If $A$ is noetherian, these conditions are equivalent to the following

(i') every finitely generated A-module has finite projective dimension

(ii') $D^{b}(A$-mod $)=A$-perf

(iii') A-perf is strongly finitely generated.

Proof. The equivalence between the first two assertions is clear, since $D^{b}(A)$ is classically generated by the $L[i]$, where $L$ runs over the $A$-modules and $i$ over $\mathbf{Z}$.

Put $D(A)^{f}=\left\langle\widetilde{D(A)^{c}}\right\rangle_{\infty}$. Note that the canonical functor $K^{b}(A-\operatorname{Proj}) \stackrel{\sim}{\rightarrow} D(A)^{f}$ is an equivalence. Let $C \in D(A)$. As in Proposition 6.3, one shows that $C \in D^{b}(A)$ if and only if $\operatorname{Hom}(-, C)_{\mid D(A)^{f}}$ is locally finitely presented.

Assume (iii). By Theorem 4.20, we have $C \in D(A)^{f}$ if and only if $\operatorname{Hom}(-, C)_{D(A)^{f}}$ is locally finitely presented. So, $D^{b}(A)=D(A)^{f}$ and (ii) holds.

Finally, (i) $\Rightarrow$ (iii) follows from Proposition 7.25.

The proof for the remaining assertions is similar.

Remark 7.27. For finite dimensional or commutative algebras over a perfect field, we obtained in Proposition 7.4 the better bound $\operatorname{dim} D^{b}(A$-mod $) \leq$ gldim $A$. We don't know whether such a bound holds under the assumption of Proposition 7.25 . 
The construction of Proposition 7.25 is not optimal when $A$ is hereditary, since the $D_{i}$ 's in Proposition 7.23 are then zero, i.e., every object of $D^{b}(A)$ is isomorphic to a direct sums of complexes concentrated in one degree. We get then the following result.

Proposition 7.28. Let $A$ be a hereditary ring. Then, $D^{b}(A)=\langle\widetilde{A}\rangle_{2}$.

Assume now $A$ is noetherian. Then, $D^{b}(A$-mod $)=\langle A\rangle_{2}$.

Remark 7.29. Proposition 7.28 generalizes easily to quasi-hereditary algebras. Let $\mathcal{C}$ be a highest weight category over a field $k$ with weight poset $\Lambda$ (i.e., the category of finitely generated modules over a quasi-hereditary algebra). Then, there is a decomposition $D^{b}(\mathcal{C})=\mathcal{I}_{1} \diamond \cdots \diamond \mathcal{I}_{d}$ such that $\mathcal{I}_{i} \simeq D^{b}\left(k^{n_{i}}\right.$-mod) for some $n_{i}$ and where $d$ is the maximal $i$ such that there is $\lambda_{1}<\cdots<\lambda_{i} \in \Lambda$ [CPS, Theorem 3.9]. It follows from Lemma 3.4 that $\operatorname{dim} D^{b}(\mathcal{C})<d$.

Remark 7.30. It would interesting to classify algebraic triangulated categories of dimension 1. Which differential graded / finite dimensional algebras can have such a derived category ? This relates to work on quasi-tilted algebras.

7.2.3. The following Lemma is related to the non-commutative [BoVdB, Lemma 4.2.4].

Lemma 7.31. Let $X$ be a quasi-projective scheme over a field and $\mathcal{L}$ an ample sheaf. Then, there are $r, l \geq 0$ such that for any $n \in \mathbf{Z}$, we have $\mathcal{L}^{\otimes n} \in \operatorname{add}\left(\{G[i]\}_{|i| \leq r}\right)^{* l}$, where $G=$ $\mathcal{L}^{\otimes-r} \oplus \mathcal{L}^{\otimes-r+1} \oplus \cdots \oplus \mathcal{L}^{\otimes r}$. If $X$ is regular, then we can take $l=1+\operatorname{dim} X$.

Proof. Pick $s>0$ such that $\mathcal{L}^{\otimes s}$ is very ample and let $i: X \rightarrow \mathbf{P}^{N}$ be a corresponding immersion (i.e., $\mathcal{L}^{\otimes s} \simeq i^{*} \mathcal{O}(1)$ ). Beilinson's resolution of the diagonal (cf example 7.7) shows that for every $i<0$, there is an exact sequence of vector bundles on $\mathbf{P}^{N}$

$$
0 \rightarrow \mathcal{O}(i) \rightarrow \mathcal{O} \otimes V_{0} \rightarrow \mathcal{O}(1) \otimes V_{1} \rightarrow \cdots \rightarrow \mathcal{O}(N) \otimes V_{N} \rightarrow 0
$$

where $V_{0}, \ldots, V_{N}$ are finite dimensional vector spaces. By restriction to $X$, we obtain an exact sequence

$$
0 \rightarrow \mathcal{L}^{\otimes s i} \stackrel{f^{-1}}{\longrightarrow} \mathcal{O} \otimes V_{0} \stackrel{f^{0}}{\longrightarrow} \mathcal{L}^{\otimes s} \otimes V_{1} \stackrel{f^{1}}{\longrightarrow} \cdots \stackrel{f^{N-1}}{\longrightarrow} \mathcal{L}^{\otimes s N} \otimes V_{N} \rightarrow 0
$$

We get a similar exact sequence for $i>0$ by dualizing. This shows the first part of the Lemma with $l=N+1$.

Assume now $X$ is regular of dimension $d$. Then, $\operatorname{Ext}^{d+1}\left(M, \mathcal{L}^{\otimes s i}\right)=0$, where $M=\operatorname{coker} f^{d-1}$. Consequently, $\mathcal{L}^{\otimes s i}$ is a direct summand of the complex

$$
0 \rightarrow \mathcal{O} \otimes V_{0} \stackrel{f^{0}}{\longrightarrow} \mathcal{L}^{\otimes s} \otimes V_{1} \stackrel{f^{1}}{\longrightarrow} \cdots \stackrel{f^{d-1}}{\longrightarrow} \mathcal{L}^{\otimes s d} \otimes V_{d} \rightarrow 0 .
$$

Dualizing, we see that, for $i>0$, then $\mathcal{L}^{\otimes s i}$ is a direct summand of a complex

$$
0 \rightarrow \mathcal{L}^{\otimes-s d} \otimes V_{d} \rightarrow \cdots \rightarrow \mathcal{L}^{\otimes-s} \otimes V_{1} \rightarrow \mathcal{O} \otimes V_{0} \rightarrow 0 .
$$

The Lemma follows.

Proposition 7.32. Let $X$ be a regular quasi-projective scheme over a field and $\mathcal{L}$ an ample sheaf. Then, $D^{b}(X$-qcoh $)=\langle\widetilde{G}\rangle_{2(1+\operatorname{dim} X)^{2}}$ and $D^{b}(X$-coh $)=\langle G\rangle_{2(1+\operatorname{dim} X)^{2}}$ for some $r>0$, where $G=\mathcal{L}^{\otimes-r} \oplus \cdots \oplus \mathcal{L}^{\otimes r}$. In particular, $\operatorname{dim} D^{b}(X$-coh $) \leq 2(1+\operatorname{dim} X)^{2}-1$.

Proof. By Lemma 7.31 , there is $r>0$ such that $\overline{\operatorname{add}}\left(\left\{\mathcal{L}^{\otimes i}\right\}_{i \in \mathbf{Z}}\right) \subset\langle\widetilde{G}\rangle_{1+\operatorname{dim} X}$ for all $i$, where $G=\mathcal{L}^{\otimes-r} \oplus \cdots \oplus \mathcal{L}^{\otimes r}$. Let $C \in D^{b}(X$-qcoh). Up to isomorphism, we can assume $C$ is a bounded complex with terms in $\overline{\operatorname{add}}\left(\left\{\mathcal{L}^{\otimes i}\right\}_{i \in \mathbf{Z}}\right)$, because $X$ is regular. Now, proceeding as in the proof of Proposition 7.25 , we get $\left.C \in \overline{\operatorname{add}}\left(\left\{\mathcal{L}^{\otimes i}\right\}_{i \in \mathbf{Z}}\right)\right\rangle_{2+2 \operatorname{dim} X}$. 
In the case of a curve, we have a slightly better (though probably not optimal) result.

Proposition 7.33. Let $X$ be a regular quasi-projective curve over a field. Then, $\operatorname{dim} D^{b}(X$-coh $) \leq$ 3 .

Lemma 7.34. Let $X$ be a separated scheme of finite type over $k$ and $U$ an open subscheme of $X$. We have $\operatorname{dim} D^{b}(U$-coh $) \leq \operatorname{dim} D^{b}(X$-coh $)$.

Proof. Lemma 3.3 gives the result, via the exact sequence $0 \rightarrow D_{X-U}^{b}(X$-coh $) \rightarrow D^{b}(X$-coh $) \rightarrow$ $D^{b}(U-\mathrm{coh}) \rightarrow 0$,

Proposition 7.35. Let $X$ be a quasi-projective scheme over $k$. Then, the following assertions are equivalent

(i) $X$ is regular

(ii) every object of $D^{b}(X$-qcoh) is isomorphic to a bounded complex of locally free sheaves

(iii) $D^{b}(X$-coh $)=X$-perf

(iv) $X$-perf is strongly finitely generated

Proof. It is clear that (ii) $\Rightarrow$ (i) and (iii) $\Rightarrow$ (i).

By Proposition 7.32, we have (i) $\Rightarrow$ (ii)-(iv).

Assume (iv). Since $X$-perf is strongly finitely generated, it follows from Lemmas 3.2 and 3.3 that $U$-perf is strongly finitely generated for any affine open $U$ of $X$ because the restriction functor $X$-perf $\rightarrow U$-perf has dense image (Theorem 5.3). So, $U$ is regular by Proposition 7.26, hence $X$ is regular. So, (iv) $\Rightarrow(\mathrm{i})$.

\subsection{Nilpotent ideals.}

Lemma 7.36. Let $A$ be a noetherian ring and $I$ a nilpotent (two-sided) ideal of $A$ with $I^{r}=0$. Let $M \in D^{b}((A / I)$-mod $)$ such that $D^{b}((A / I)$-mod $)=\langle M\rangle_{n}$. Then, $D^{b}(A$-mod $)=\langle M\rangle_{r n}$.

In particular, $\operatorname{dim} D^{b}(A$-mod $) \leq r\left(1+\operatorname{dim} D^{b}((A / I)\right.$-mod $\left.)\right)-1$.

Proof. Let $C$ be a bounded complex of finitely generated $A$-modules. We have a filtration $0=I^{r} C \subset I^{r-1} C \subset \cdots \subset I C \subset C$ whose successive quotients are bounded complexes of finitely generated $(A / I)$-modules and the Lemma follows.

We have a geometric version as well.

Lemma 7.37. Let $X$ be a separated noetherian scheme, $\mathcal{I}$ a nilpotent ideal sheaf with $\mathcal{I}^{r}=0$ and $i: Z \rightarrow X$ the corresponding closed immersion. Let $M \in D^{b}(Z$-coh $)$ such that $D^{b}(Z$-coh $)=$ $\langle M\rangle_{n}$. Then, $D^{b}(X-\mathrm{coh})=\left\langle i_{*} M\right\rangle_{r n}$. Similarly, for $M \in D^{b}(Z$-qcoh $)$ such that $D^{b}(Z$-qcoh $)=$ $\langle\widetilde{M}\rangle_{n}$, then $D^{b}(X$-qcoh $)=\left\langle\widetilde{i_{*} M}\right\rangle_{r n}$.

In particular, $\operatorname{dim} D^{b}(X-\operatorname{coh}) \leq r\left(1+\operatorname{dim} D^{b}(Z\right.$-coh $\left.)\right)-1$.

For an artinian ring $A$, the Loewy length $\operatorname{ll}(A)$ of $A$ is the smallest integer $i$ such that $J(A)^{i}=0$, where $J(A)$ is the Jacobson radical of $A$.

From Lemma 7.36, we deduce

Proposition 7.38. Let $A$ be an artinian ring. Then, $D^{b}(A$-mod $)=\langle A / J(A)\rangle_{11(A)}$. In particular, $\operatorname{dim} D^{b}(A$-mod $) \leq \operatorname{ll}(A)-1$.

7.4. Finiteness for derived categories of coherent sheaves. Let $k$ be a field. 
7.4.1. The following Theorem is due to Kontsevich, Bondal and Van den Bergh for $X$ non singular BoVdB, Theorem 3.1.4].

Theorem 7.39. Let $X$ be a separated scheme of finite type over a perfect field $k$. Then, there is $E \in D^{b}(X$-coh) and $d \in \mathbf{N}$ such that

$$
D(X \text {-qcoh })=\langle\bar{E}\rangle_{d}, D^{b}(X-\mathrm{qcoh})=\langle\widetilde{E}\rangle_{d} \text { and } D^{b}(X \text {-coh })=\langle E\rangle_{d} .
$$

In particular, $\operatorname{dim} D^{b}(X-\operatorname{coh})<\infty$.

Let us explain how the Theorem will be proved. It is enough to consider the case where $X$ is reduced. Then, the structure sheaf of the diagonal is a direct summand of a perfect complex up to a complex supported on $Z \times X$, where $Z$ is a closed subscheme with smooth dense complement. We conclude by induction by applying the Theorem to $Z$.

Let us start with two Lemmas.

Lemma 7.40. Let $A$ and $B$ be two finitely generated commutative $k$-algebras, where $k$ is perfect. Let $M$ be a finitely generated $(B \otimes A)$-module and $\cdots \rightarrow P^{-1} \stackrel{d^{-2}}{\longrightarrow} P^{0} \stackrel{d^{-1}}{\longrightarrow} M \stackrel{d^{0}}{\longrightarrow} 0$ be an exact complex with $P^{i}$ finitely generated and projective.

If $M$ is flat as an $A$-module and $B$ is regular of dimension $n$, then $\operatorname{ker} d^{-n}$ is a projective $(B \otimes A)$-module.

Proof. Let $i \geq 1, \mathfrak{m}$ a maximal ideal of $A$ and $\mathfrak{n}$ a maximal ideal of $B$. We have

$$
\operatorname{Tor}_{i}^{B \otimes A}\left(\operatorname{ker} d^{-n}, B / \mathfrak{n} \otimes A / \mathfrak{m}\right) \simeq \operatorname{Tor}_{n+i}^{B \otimes A}(M, B / \mathfrak{n} \otimes A / \mathfrak{m}) \simeq \operatorname{Tor}_{n+i}^{B}\left(M \otimes_{A} A / \mathfrak{m}, B / \mathfrak{n}\right)=0
$$

since $B$ is regular with dimension $n$. It follows that $\operatorname{ker} d^{-n}$ is projective (cf Ma, $\S 18$, Lemma $5])$.

Lemma 7.41. Let $X$ be a separated noetherian scheme and $Z$ a closed subscheme of $X$, given by the ideal sheaf $\mathcal{I}$ of $\mathcal{O}_{X}$. For $n \geq 1$, let $Z_{n}$ be the closed subscheme of $X$ with ideal sheaf $\mathcal{I}^{n}$ and $i_{n}: Z_{n} \rightarrow X$ the corresponding immersion.

Then, given $C \in D_{Z}^{b}(X$-coh $)$, there is $n \geq 1$ and $C_{n} \in D^{b}\left(Z_{n}\right.$-coh $)$ such that $C \simeq i_{n *} C_{n}$.

Proof. Let $\mathcal{F}$ be a coherent sheaf on $X$ supported by $Z$. Then, $\mathcal{I}^{n} \mathcal{F}=0$ for some $n$ and it follows that $\mathcal{F} \stackrel{\sim}{\rightarrow} i_{n *}\left(i_{n}^{*} \mathcal{F}\right)$. More generally, a bounded complex of coherent sheaves on $X$ that are supported by $Z$ is isomorphic to the image under $i_{n *}$ of a bounded complex of coherent sheaves on $Z_{n}$ for some $n$.

Let $\mathcal{F}$ be a coherent sheaf on $X$. Let $\mathcal{F}_{Z}$ be the subsheaf of $\mathcal{F}$ of sections supported by $Z$. By Artin-Rees' Theorem [Ma, $\S 11$.C Theorem 15], there is an integer $r$ such that $\left(\mathcal{I}^{m} \mathcal{F}\right) \cap \mathcal{F}_{Z}=$ $\mathcal{I}^{m-r}\left(\mathcal{I}^{r} \cap \mathcal{F}_{Z}\right)$ for $m \geq r$. Since $\mathcal{F}_{Z}$ is a coherent sheaf supported by $Z$, there is an integer $d$ such that $\mathcal{I}^{d} \mathcal{F}_{Z}=0$. So, $\left(\mathcal{I}^{r+d} \mathcal{F}\right) \cap \mathcal{F}_{Z}=0$. It follows that the canonical map $\mathcal{F}_{Z} \rightarrow \mathcal{F} /\left(\mathcal{I}^{r+d} \mathcal{F}\right)$ is injective.

We prove now the Lemma by induction on the number of terms of $C$ that are not supported by $Z$.

Let $C=0 \rightarrow C^{r} \stackrel{d^{r}}{\longrightarrow} \cdots \stackrel{d^{s-1}}{\longrightarrow} C^{s} \rightarrow 0$ be a complex of coherent sheaves on $X$ with cohomology supported by $Z$ and take $i$ minimal such that $C^{i}$ is not supported by $Z$.

Since $C^{i-1}$ and $H^{i}(C)$ are supported by $Z$, it follows that ker $d^{i}$ is supported by $Z$. So, there is an integer $n$ such that the canonical map ker $d^{i} \rightarrow C^{i} /\left(\mathcal{I}^{n} C^{i}\right)$ is injective. Let $R$ be the subcomplex of $C$ with non zero terms $R^{i}=\mathcal{I}^{n} C^{i}$ and $R^{i+1}=d^{i}\left(\mathcal{I}^{n} C^{i}\right)$ - a complex homotopy 
equivalent to 0 . Let $D=C / R$. Then, the canonical map $C \rightarrow D$ is a quasi-isomorphism. By induction, $D$ is quasi-isomorphic to a complex of coherent sheaves on $Z_{n}$ for some $n$ and the Lemma follows.

Proof of Theorem 7.39. We have $D^{b}(X \text {-qcoh })^{c}=D^{b}(X$-coh) (Corollary 6.16). So, the assertion about $D^{b}\left(X\right.$-coh) follows immediately from the one about $D^{b}(X$-qcoh $)$ by Corollary 3.13. We give the proof only for the case $D^{b}(X$-qcoh $)$, the case of $D(X$-qcoh $)$ is similar and easier. By Lemma 7.37, it is enough to prove the Theorem for $X$ reduced.

Assume $X$ is reduced and let $d$ be its dimension. We now prove the Theorem by induction on $d$ (the case $d=0$ is trivial).

Let $U$ be a smooth dense open subscheme of $X$. The structure sheaf $\mathcal{O}_{\Delta U}$ of the diagonal $\Delta U$ in $U \times X$ is a perfect complex by Lemma 7.40. By Thomason and Trobaugh's localization Theorem (Theorem 5.3), there is a perfect complex $C$ on $X \times X$ and a morphism $f: C \rightarrow$ $\mathcal{O}_{\Delta X} \oplus \mathcal{O}_{\Delta X}[1]$ whose restriction to $U \times X$ is an isomorphism. Let $G$ be a compact generator for $D(X$-qcoh $)$. Then, $G \otimes G$ is a compact generator for $D((X \times X)$-qcoh $)$ BoVdB, Lemma 3.4.1]. So, there is $r$ such that $C \in\langle G \otimes G\rangle_{r}$ by Theorem 4.22 (3).

Let $D$ be the cone of $f$. Then, $H^{*}(D)$ is supported by $Z \times X$, where $Z=X-U$. It follows that there is a closed subscheme $Z^{\prime}$ of $X$ with underlying closed subspace $Z$, a bounded complex $D^{\prime}$ of coherent $\mathcal{O}_{Z^{\prime} \times X^{-}}$-modules and an isomorphism $(i \times \text { id })_{*} D^{\prime} \stackrel{\sim}{\rightarrow} D$ in $D^{b}((X \times X)$-coh), where $i: Z^{\prime} \rightarrow X$ is the closed immersion (Lemma 7.41). By induction, there is $M \in D^{b}\left(Z^{\prime}\right.$-coh) and an integer $l$ such that $D^{b}\left(Z^{\prime}\right.$-qcoh $)=\langle\widetilde{M}\rangle_{l}$.

Let $p_{1}$ and $p_{2}$ be the first and second projections $X \times X \rightarrow X$ and $\pi: Z^{\prime} \times X \rightarrow Z^{\prime}$ be the first projection. Let $\mathcal{F} \in D^{b}(X$-qcoh). We have a distinguished triangle

$$
R p_{1 *}\left(C \otimes^{\mathbf{L}} p_{2}^{*} \mathcal{F}\right) \rightarrow \mathcal{F} \oplus \mathcal{F}[1] \rightarrow R p_{1 *}\left(D \otimes^{\mathbf{L}} p_{2}^{*} \mathcal{F}\right) \rightsquigarrow .
$$

Since $C$ is perfect, we have $C \otimes^{\mathbf{L}} p_{2}^{*} \mathcal{F} \in D^{b}\left((X \otimes X)\right.$-qcoh), hence $R p_{1 *}\left(C \otimes{ }^{\mathbf{L}} p_{2}^{*} \mathcal{F}\right)$ has bounded cohomology. It follows that $R p_{1 *}\left(D \otimes^{\mathbf{L}} p_{2}^{*} \mathcal{F}\right)$ has bounded cohomology as well. We have

$$
\left.R p_{1 *}\left(D \otimes \mathbf{L} p_{2}^{*} \mathcal{F}\right) \simeq R p_{1 *}(i \times \mathrm{id})_{*}\left(D^{\prime} \otimes^{\mathbf{L}} \mathbf{L}(i \times \mathrm{id})^{*} p_{2}^{*} \mathcal{F}\right)\right) \simeq i_{*} R \pi_{*}\left(D^{\prime} \otimes^{\mathbf{L}}\left(\mathcal{O}_{Z^{\prime}} \otimes \mathcal{F}\right)\right)
$$

Note that $R \pi_{*}\left(D^{\prime} \otimes \mathbf{L}\left(\mathcal{O}_{Z^{\prime}} \otimes \mathcal{F}\right)\right)$ is an element of $D^{b}\left(Z^{\prime}\right.$-qcoh). So, $R p_{1 *}\left(D \otimes \mathbf{L} p_{2}^{*} \mathcal{F}\right) \in\left\langle\widetilde{i_{*} M}\right\rangle_{l}$.

We have $(G \otimes G) \otimes^{\mathbf{L}} p_{2}^{*} \mathcal{F} \simeq G \otimes\left(G \otimes^{\mathbf{L}} \mathcal{F}\right)$, hence $R p_{1 *}\left((G \otimes G) \otimes \mathbf{L} p_{2}^{*} \mathcal{F}\right) \simeq G \otimes R \Gamma\left(G \otimes^{\mathbf{L}} \mathcal{F}\right) \in\langle\widetilde{G}\rangle$ (note this has bounded cohomology). So, $R p_{1 *}\left(C \otimes \mathbf{L} p_{2}^{*} \mathcal{F}\right) \in\langle\widetilde{G}\rangle_{r}$.

Finally, $\mathcal{F} \in\left\langle i_{*} \widetilde{M \oplus} G\right\rangle_{l+r}$ and we are done.

Remark 7.42. In Theorem 7.39, one can require $E$ to be a sheaf (consider $\bigoplus_{i} H^{i}(E)$ ).

Remark 7.43. Note that when $X$ is smooth, then the proof shows the stronger functorial result as in $\$ 3.2 .2$ - this is Kontsevich's result. This stronger property does not hold in general for singular $X$, cf the case $X=\operatorname{Spec} k[x] / x^{2}$.

Remark 7.44. Theorem 7.39 does not extend to the derived categories $D_{Z}(X$-coh). For example, $D_{\{0\}}^{b}\left(\mathbf{A}_{k}^{1}\right.$-coh) is not strongly finitely generated.

Remark 7.45. Note that the proof works under the weaker assumption that $X$ is a separated scheme of finite type over $k$ and the residue fields at closed points are separable extensions of $k$. 
We don't know how to bound the dimension of $D^{b}(X$-coh) for singular $X$. When $X$ is zero dimensional, then $\operatorname{dim} D^{b}(X-\operatorname{coh})=0$ if and only if $X$ is smooth.

We don't know whether the inequality $\operatorname{dim} D^{b}((X \times Y)$-coh $) \leq \operatorname{dim} D^{b}(X$-coh $)+\operatorname{dim} D^{b}(Y$-coh $)$ holds for $X, Y$ separated schemes of finite type over a perfect field.

Last but not least, we don't know a single case where $X$ is smooth and $\operatorname{dim} D^{b}(X$-coh $)>$ $\operatorname{dim} X$. For example, we don't know whether $\operatorname{dim} D^{b}(X$-coh $)=1$ or 2 for $X$ an elliptic curve over an algebraically closed field.

We now deduce that stable derived categories are strongly finitely generated as well.

Corollary 7.46. Let $X$ be a separated scheme of finite type over a perfect field $k$ and $\mathcal{T}=$ $D^{b}(X$-coh $) / X$-perf. Then, $\operatorname{dim} \mathcal{T}<\infty$.

Assume $X$ is Gorenstein, has enough locally free sheaves and its singular locus is complete. Then $\mathcal{T}$ is Ext-finite, hence every locally finite cohomological functor is representable.

Proof. The first statement is an immediate consequence of Theorem 7.39 and Lemma 3.3. The fact that $\mathcal{T}$ is Ext-finite is $[\mathrm{Or}$, Corollary 2.24 and its proof] and the representability statement is Corollary 4.18 .

7.4.2. Let $X$ be a projective scheme over a field $k$. Given $C \in X$-perf and $D \in D^{b}(X$-coh), then $\operatorname{dim} \bigoplus_{i \in \mathbf{Z}} \operatorname{Hom}(C, D[i])<\infty$.

The following result is given by BoVdB, Theorem A.1].

Lemma 7.47. An object $D \in D(X)$ is in $D^{b}(X$-coh) if and only if for all $C \in X$-perf, we have $\operatorname{dim} \bigoplus_{i \in \mathbf{Z}} \operatorname{Hom}(C, D[i])<\infty$.

Proof. The first implication has been recalled before.

Let $D \in D(X)$ such that $\operatorname{Hom}(-, D)_{\mid X \text {-perf }}$ is locally finite. Then, $\operatorname{Hom}(-, D)_{\mid X \text {-perf }}$ is locally finitely presented (Proposition 4.9), hence $D \in D^{b}(X$-coh) (Proposition 6.12).

Proposition 7.48. There is a fully faithful functor $S: X$-perf $\rightarrow D^{b}(X$-coh) and bifunctorial isomorphisms

$$
\operatorname{Hom}(C, D)^{*} \stackrel{\sim}{\rightarrow} \operatorname{Hom}(D, S(C))
$$

for $C \in X$-perf and $D \in D(X)$.

Proof. The category $D(X)$ is cocomplete and has a compact generator (Theorem 6.8). Corollary 4.23 shows the existence of a functor $S: X$-perf $\rightarrow D(X)$.

By Lemma 7.47, if $C \in X$-perf, then $S(C) \in D^{b}(X$-coh).

We can now prove a "dual version" of Lemma 7.47 :

Lemma 7.49. An object $C \in D(X)$ is in $X$-perf if and only if for all $D \in D^{b}(X$-coh), we have $\operatorname{dim} \bigoplus_{i \in \mathbf{Z}} \operatorname{Hom}(C, D[i])<\infty$.

Proof. The first implication has been recalled before.

Let $C \in D(X)$ such that for all $D \in D^{b}(X$-coh $)$, we have $\operatorname{dim} \bigoplus_{i \in \mathbf{Z}} \operatorname{Hom}(C, D[i])<\infty$. Let $D^{\prime} \in X$-perf. Then,

$$
\operatorname{Hom}\left(D^{\prime}, C[i]\right)^{*} \stackrel{\sim}{\rightarrow} \operatorname{Hom}\left(C, S\left(D^{\prime}\right)[i]\right),
$$

hence $\operatorname{dim} \bigoplus_{i \in \mathbf{Z}} \operatorname{Hom}\left(D^{\prime}, C[i]\right)<\infty$. It follows from Lemma 7.47 that $C \in D^{b}(X$-coh). 
Let $x$ be a closed point of $X$. We have $\operatorname{dim} \bigoplus_{i} \operatorname{Hom}\left(C, \mathcal{O}_{\{x\}}[i]\right)=\operatorname{dim} \bigoplus_{i} \operatorname{Hom}\left(C_{x}, \mathcal{O}_{\{x\}}[i]\right)<$ $\infty$. This shows that $C_{x}$ is a perfect complex of $\mathcal{O}_{x}$-modules. Since $C \in D^{b}(X$-coh), we deduce that $C$ is perfect.

The following result was conjectured by Bondal — the first statement is BoVdB, Theorem A.1].

Corollary 7.50. Let $X$ be a projective scheme over a perfect field $k$.

(i) Every locally finite cohomological functor $(X \text {-perf })^{\circ} \rightarrow k$-mod is representable by an object of $D^{b}(X$-coh).

(ii) Every locally finite cohomological functor $D^{b}(X$-coh $) \rightarrow k$-mod is representable by an object of $X$-perf.

Proof. By Remark 4.30, a finite cohomological functor $(X \text {-perf })^{\circ} \rightarrow k$-mod is representable by an object of $D(X)$ and Lemma 7.47 says that the object must be in $D^{b}(X$-coh). This shows (i).

By Theorem 7.39, $D^{b}(X \text {-coh })^{\circ}$ is strongly finitely generated. So, Proposition 4.9 and Corollary 4.17 show that every locally finite cohomological functor $D^{b}(X$-coh $) \rightarrow k$-mod is representable by an object of $D^{b}(X$-coh) and Lemma 7.49 says that the object must be in $X$-perf. This shows (ii).

Remark 7.51. Similar results should hold for $X$ quasi-projective, with $D^{b}(X$-coh) replaced by its full subcategory of objects with compact support.

\section{Applications to Finite dimensional algebras}

\subsection{Auslander's representation dimension.}

8.1.1. Let $\mathcal{A}$ be an abelian category.

Definition 8.1. The (Auslander) representation dimension repdim $\mathcal{A}$ is the smallest integer $i \geq 2$ such that there is an object $M \in \mathcal{A}$ with the property that given any $L \in \mathcal{A}$,

(a) there is an exact sequence

$$
0 \rightarrow M^{-i+2} \rightarrow M^{-i+3} \rightarrow \cdots \rightarrow M^{0} \rightarrow L \rightarrow 0
$$

with $M^{j} \in \operatorname{add}(M)$ such that the sequence

$$
\begin{aligned}
& 0 \rightarrow \operatorname{Hom}\left(M, M^{-i+2}\right) \rightarrow \operatorname{Hom}\left(M, M^{-i+3}\right) \rightarrow \cdots \rightarrow \operatorname{Hom}\left(M, M^{0}\right) \rightarrow \operatorname{Hom}(M, L) \rightarrow 0 \\
& \quad i s \text { exact }
\end{aligned}
$$

(b) there is an exact sequence

$$
0 \rightarrow L \rightarrow M^{\prime 0} \rightarrow M^{\prime 1} \rightarrow \cdots \rightarrow M^{\prime i-2} \rightarrow 0
$$

with $M^{\prime j} \in \operatorname{add}(M)$ such that the sequence

$$
0 \rightarrow \operatorname{Hom}\left(M^{\prime i-2}, M\right) \rightarrow \cdots \rightarrow \operatorname{Hom}\left(M^{\prime 1}, M\right) \rightarrow \operatorname{Hom}\left(M^{\prime 0}, M\right) \rightarrow \operatorname{Hom}(L, M) \rightarrow 0
$$

is exact. 
An object $M$ that realizes the minimal $i$ is called an Auslander generator.

Note that either condition (a) or (b) implies that gldim $\operatorname{End}_{\mathcal{A}}(M) \leq i$, and gldim $\operatorname{End}_{\mathcal{A}}(M)=$ $i$ if $M$ is an Auslander generator (cf e.g. ErHolyso, Lemma 2.1]). Note also that if condition (a) (resp. (b)) hold for every $L$ in a dense subcategory $\mathcal{I}$ of $\mathcal{A}$, then, it holds for every object of $\mathcal{A}$.

Note that repdim $\mathcal{A}=2$ if and only if $\mathcal{A}$ has only finitely many isomorphism classes of indecomposable objects. Note also that $\operatorname{repdim} \mathcal{A}=\operatorname{repdim} \mathcal{A}^{\circ}$.

8.1.2. Take $\mathcal{A}=A$-mod, where $A$ is a finite dimensional algebra over a field. Then, we write $\operatorname{repdim}(A)$ for $\operatorname{repdim}(A$-mod).

Let $M \in \mathcal{A}$ and $i \geq 2$. If $M$ satisfies (a) of Definition 8.1, then it contains a projective generator as a direct summand (take $L=A$ ). More generally, the following are equivalent

- $M$ satisfies (a) of Definition 8.1 and $M$ contains an injective cogenerator as a direct summand

- $M$ satisfies (b) of Definition 8.1 and $M$ contains a projective generator as a direct summand

- $M$ satisfies (a) and (b) of Definition 8.1.

So, the definition of representation dimension given here coincides with Auslander's original definition (cf [Au, §III.3] and [ErHolySa, Lemma 2.1]) when $A$ is not semi-simple. When $A$ is semi-simple, Auslander assigns the representation dimension 0 whereas we define it to be 2 here. Iyama has shown [Iy] that the representation dimension of a finite dimensional algebra is finite.

Various classes of algebras with representation dimension 3 have been found : algebras with radical square zero [Au, §III.5, Proposition p.56], hereditary algebras [Au, §III.5, Proposition p.58] and more generally stably hereditary algebras [Xi, Theorem 3.5], special biserial algebras ErHoIySd, local algebras of quaternion type $\mathrm{Ho}$.

8.1.3. One can weaken the requirements in the definition of the representation dimension as follows :

Definition 8.2. The weak (resp. left weak, resp. right weak) representation dimension of $\mathcal{A}$, denoted by $\operatorname{wrepdim}(\mathcal{A})$ (resp. $\operatorname{lwrepdim}(\mathcal{A})$, resp. $\operatorname{rwrepdim}(\mathcal{A})$ ) is the smallest integer $i \geq 2$ such that there is an object $M \in \mathcal{A}$ with the property that given any $L \in \mathcal{A}$, there is a bounded complex $C=0 \rightarrow C^{r} \rightarrow \cdots \rightarrow C^{s} \rightarrow 0$ of $\operatorname{add}(M)$ with

- $L$ isomorphic to a direct summand of $H^{0}(C)$

- $H^{d}(C)=0$ for $d \neq 0$ and

- $s-r \leq i-2$ (resp. and $C^{d}=0$ for $d>0$, resp. and $C^{d}=0$ for $\left.d<0\right)$.

Note that lwrepdim $\mathcal{A}=\operatorname{rwrepdim} \mathcal{A}^{\circ}, \operatorname{wrepdim} \mathcal{A}=\operatorname{wrepdim} \mathcal{A}^{\circ}$,

$\inf \{\operatorname{lwrepdim} \mathcal{A}, \operatorname{rwrepdim} \mathcal{A}\} \geq \operatorname{wrepdim} \mathcal{A}$ and $\operatorname{repdim} \mathcal{A} \geq \sup \{\operatorname{lwrepdim} \mathcal{A}, \operatorname{rwrepdim} \mathcal{A}\}$.

Proposition 8.3. We have $\operatorname{dim} D^{b}(A$-mod $) \leq \operatorname{repdim}(A)$ and $\operatorname{dim}\left(D^{b}(A\right.$-mod $) / A$-perf $) \leq$ wrepdim $(A)-2$.

Proof. Let $M$ be an Auslander generator for $A$-mod. Let $C$ be a bounded complex of objects of $\operatorname{add}(M)$. Let $L \in A$-mod and let $D$ be a bounded complex of add $(M)$ together with a 
map $f: D \rightarrow L$ such that $\operatorname{Hom}(M, f)$ is a quasi-isomorphism (in particular, $f$ is a quasiisomorphism). Then, $\operatorname{Hom}^{\bullet}(C, f): \operatorname{Hom}^{\bullet}(C, D) \rightarrow \operatorname{Hom}^{\bullet}(C, L)$ is a quasi-isomorphism, hence $\operatorname{Hom}(C, f): \operatorname{Hom}_{K^{b}(A)}(C, D) \rightarrow \operatorname{Hom}_{K^{b}(A)}(C, L)$ is an isomorphism.

It follows by induction that every bounded complex of $A$-mod is quasi-isomorphic to a bounded complex of add $(M)$, i.e., the canonical functor $K^{b}(\operatorname{add}(M)) \rightarrow D^{b}(A$-mod $)$ is essentially surjective. We have equivalences $K^{b}(\operatorname{End}(M)$-proj $) \stackrel{\sim}{\rightarrow} D^{b}(\operatorname{End}(M))$ and $K^{b}(\operatorname{End}(M)$-proj $) \stackrel{\sim}{\rightarrow}$ $K^{b}(\operatorname{add}(M))$ and $\operatorname{dim} D^{b}(\operatorname{End}(M)) \leq \operatorname{gldim} \operatorname{End}(M)$ by Proposition 7.4. So, $\operatorname{dim} D^{b}(A-\bmod ) \leq$ $\operatorname{repdim}(A)$ by Lemma 3.3 .

Let $n=\operatorname{wrepdim} A$. There is $N \in A$-mod with the property that given $L \in A$-mod, there is a bounded complex of $\operatorname{add}(N) C=0 \rightarrow C^{r} \rightarrow \cdots \rightarrow C^{s} \rightarrow 0$ with $H^{i}(C)=0$ for $i \neq 0$, $L$ is a direct summand of $H^{0}(C)$ and $s-r \leq n-2$. Then $L \in\left\langle C^{s}\right\rangle \diamond \cdots \diamond\left\langle C^{r}\right\rangle$. Every object of $D^{b}(A$-mod) $/ A$-perf is isomorphic to an object $L[r]$ for some $L \in A$-mod and $r \in \mathbf{Z}$. Consequently, $D^{b}(A$-mod $) / A$-perf $=\langle M\rangle_{n-1}$.

In order to obtain lower bounds for the representation dimension of certain algebras, we will actually construct lower bounds for the weak representation dimension.

Remark 8.4. Note that the representation dimension as well as the invariants of Definition 8.2 are not invariant by derived equivalence (consider for instance a derived equivalence between an algebra with finite representation type and an algebra with infinite representation type).

Remark 8.5. All the definitions given here for abelian categories make sense for exact categories.

\subsection{Stable categories of self-injective algebras. Let $k$ be a field.}

8.2.1. For $A$ a self-injective finite dimensional $k$-algebra, we denote by $A$-stab the stable category of $A$. This is the quotient of the additive category $A$-mod by the additive subcategory $A$-proj. The canonical functor $A$-mod $\rightarrow D^{b}(A$-mod) induces an equivalence $A$-stab $\stackrel{\sim}{\rightarrow}$ $D^{b}(A$-mod) $/ A$-perf ( $\mathbb{K e V 0}$, Example 2.3] and $\mathbb{R} i$, Theorem 2.1]). This provides $A$-stab with a structure of triangulated category. Recall that $\operatorname{ll}(A)$ denotes the Loewy length of $A$ (cf $\$(7.3)$.

Proposition 8.6. Let $A$ be a non-semisimple self-injective algebra. Then,

$$
\mathrm{ll}(A) \geq \operatorname{repdim} A \geq \operatorname{wrepdim} A \geq 2+\operatorname{dim} A \text {-stab. }
$$

Proof. The first inequality is [Au, $\S$ III.5, Proposition p.55] (use $M=A \oplus A / J(A) \oplus A / J(A)^{2} \oplus$ ...). The second inequality is trivial (cf $\oint 8.1 .3)$. The last inequality is given by Proposition 8.3 .

8.2.2. We study here self-injective algebras with representation dimension 3 .

Recently, various properties have been found for algebras of representation dimension 3 (cf for example $\operatorname{IgT0})$. Here is a result in this direction concerning self-injective algebras.

Note that $\operatorname{repdim} A=2$ if and only if $\operatorname{dim} A$-stab $=0$. Consequently, if $\operatorname{repdim} A=3$, then $\operatorname{dim} A$-stab $=1$ (cf Proposition 8.6).

Given $M$ an $A$-module, we denote by $\Omega M$ the kernel of a surjective map from a projective cover of $M$ to $M$ and by $\Omega^{-1} M$ the cokernel of an injective map from $M$ to an injective hull of $M$. 
Lemma 8.7. Let $A$ be a self-injective $k$-algebra and $C=0 \rightarrow C^{0} \rightarrow C^{1} \rightarrow 0$ an indecomposable complex of finitely generated $A$-modules with $H^{0}(C)=0$ and $H^{1}(C)=S$ simple. Then,

- $C^{0}$ and $C^{1}$ have no non-zero projective direct summand

- or $C^{1}$ is projective indecomposable and $C^{0} \simeq \Omega S$.

Proof. If $C^{0}$ has a non-zero projective summand $L$, then $L$ is injective and the restriction of $d=d_{C}^{0}$ to $L$ is a split injection. In particular, $C$ has a direct summand isomorphic to $0 \rightarrow L \stackrel{\text { id }}{\longrightarrow} L \rightarrow 0$, which is impossible.

Assume now that $C^{1}$ has a submodule $N$ such that $C^{1} / N$ is projective indecomposable. If $N \nsubseteq$ im $d$, then there is $P \subseteq$ im $d$ such that $C^{1}=N \oplus P$. So, $C$ has a direct summand isomorphic to $0 \rightarrow P \stackrel{\text { id }}{\longrightarrow} P \rightarrow 0$ : this is impossible. So, $C^{0} \stackrel{\sim}{\rightarrow} \operatorname{im} d=N \oplus N^{\prime}$ with $N^{\prime} \simeq \Omega S$. The indecomposability of $C$ gives $N=0$.

Lemma 8.8. Let $A$ be a self-injective k-algebra with repdim $A=3$ and $M$ an Auslander generator. Assume $\Omega^{-1} M$ has no simple direct summand. Then, the number of simple $A$ modules (up to isomorphism) is less than or equal to the number of isomorphism classes of non projective indecomposable summands of $M$.

Proof. Since repdim $A=3$, for every simple $A$-module $S$, there is an exact sequence $0 \rightarrow M_{1} \rightarrow$ $M_{0} \rightarrow S \rightarrow 0$ with $M_{0}$ and $M_{1}$ in $\operatorname{add}(M)$. By Lemma 8.7, we can assume that $M_{0}$ and $M_{1}$ have no non-zero projective direct summands. Then, we have $[S]=\left[M_{0}\right]-\left[M_{1}\right]$ in $K_{0}(A$-mod). It follows that the non-projective indecomposable summands of $M$ generate $K_{0}(A$-mod $)$.

Let $A$ and $B$ be two self-injective algebras. A stable equivalence of Morita type between $A$ and $B$ is the data of a finite dimensional $(A, B)$-bimodule $X$, projective as an $A$-module and as a right $B$-module, and of a finite dimensional $(B, A)$-bimodule $Y$, projective as a $B$-module and as a right $A$-module, such that

$$
\begin{gathered}
X \otimes_{B} Y \simeq A \oplus \text { projective as }(A, A) \text { - bimodules } \\
Y \otimes_{A} X \simeq B \oplus \text { projective as }(B, B)-\text { bimodules. }
\end{gathered}
$$

Stable equivalences of Morita type preserve the representation dimension [Xi], Theorem 4.1] :

Proposition 8.9. Let $A$ and $B$ be two self-injective $k$-algebras and $X$ be an $(A, B)$-bimodule inducing a stable equivalence between $A$ and $B$.

Let $M$ be an Auslander generator for $B$. Then, $X \otimes_{B} M$ is an Auslander generator for $A$.

In particular, $\operatorname{repdim} A=\operatorname{repdim} B$.

Proof. Given $M$ containing a progenerator as a direct summand, the property (a) (resp. (b)) for $L$ in Definition 8.1 is equivalent to the same property for $L \oplus P$, where $P$ is some fixed projective module.

Let $Y$ be a $(B, A)$-bimodule inverse to $X$. Let $V$ be an $A$-module. Then, $X \otimes_{B} Y \otimes_{A} V \simeq V \oplus P$ with $P$ projective. Starting with an exact sequence resolving $Y \otimes_{A} V$ as in (a) or (b) of Definition 8.1, we get one for $V \oplus P$ by applying $X \otimes_{B}-$ Now, applying $\operatorname{Hom}_{B}\left(X \otimes_{B} M,-\right)$ to that new exact sequence gives the same result as applying $\operatorname{Hom}_{A}\left(Y \otimes_{A} X \otimes_{B} M,-\right)$ to the original exact sequence. Since $Y \otimes_{A} X \otimes_{B} M \simeq M \oplus$ projective, we indeed get an exact sequence.

The following Proposition gives a bound for the number of non-projective simple modules of a self-injective algebra which is stably equivalent (à la Morita) to a given self-injective algebra with representation dimension 3 . 
Proposition 8.10. Let $A$ be a self-injective $k$-algebra with repdim $A=3$ and $M$ an Auslander generator. Let $B$ be a self-injective $k$-algebra. Assume there is a stable equivalence of Morita type between $A$ and $B$.

Then, the number of simple non-projective B-modules (up to isomorphism) is less than or equal to twice the number of isomorphism classes of indecomposable summands of $M$.

Proof. Replacing $B$ by a direct factor, one can assume $B$ has no simple projective module.

Let $Y$ be a $(B, A)$-bimodule inducing a stable equivalence and $N=Y \otimes_{A} M$. Then, $N$ is an Auslander generator for $B$ and $\operatorname{repdim} B=3$ (Proposition 8.9). Let $R$ be the subgroup of $K_{0}(B$-mod) generated by the classes of the non-projective indecomposable summands of $N$. Note that the rank of $R$ is at most the number of isomorphism classes of indecomposable non-projective summands of $M$.

Let $S$ be a simple $B$-module with $[S] \notin R$. There is an exact sequence $0 \rightarrow N_{1} \rightarrow N_{0} \rightarrow S \rightarrow$ 0 with $N_{0}$ and $N_{1}$ in add $(N)$ and by Lemma 8.7, $N_{0}$ is a projective cover of $S$ and $N_{1} \simeq \Omega S$. In particular, $\Omega S$ is a direct summand of $N$.

So, the number of simple $B$-modules with $[S] \notin R$ is at most the number of isomorphism classes of indecomposable non-projective summands of $N$.

Remark 8.11. This Proposition, which was the starting point of this paper, led us to investigate the existence of self-injective algebras with representation dimension greater than 3 . This Proposition is related to the problem of the equality of the number of simple non projective modules for two stably equivalent algebras.

8.2.3. The following Theorem gives the first known examples of algebras with representation dimension $>3$.

Theorem 8.12. Let $n \geq 1$ be an integer. Then, $\operatorname{dim} \Lambda\left(k^{n}\right)$-stab $=\operatorname{repdim} \Lambda\left(k^{n}\right)-2=n-1$ and $\operatorname{dim} D^{b}\left(\Lambda\left(k^{n}\right)\right.$-mod $)=n$.

Proof. Put $A=\Lambda\left(k^{n}\right)$ and $B=k\left[x_{1}, \ldots, x_{n}\right]$.

Let us recall a version of Koszul duality [Ke, §10.5, Lemma "The "exterior' case"]. We have an equivalence of triangulated categories $R \operatorname{Hom}^{\bullet}(k,-)$ between $D^{b}(A$-mod) and $\mathcal{T}$, the subcategory of the derived category of differential graded $B$-modules classically generated by $B(\mathcal{T}$ is also the subcategory of compact objects by Corollary 6.1). Note that this is a special case of $\S 3.4$, using the fact that $R \operatorname{End}^{\bullet}(k)$ is a dg algebra quasi-isomorphic to its cohomology algebra $B$. This equivalence sends $A$ to $k$, so it induces an equivalence of triangulated categories between $A$-stab and $\mathcal{T} / \mathcal{I}$, where $\mathcal{I}$ is the thick subcategory of $\mathcal{T}$ classically generated by $k$. Denote by $F: \mathcal{T} \rightarrow \mathcal{T} / \mathcal{I}$ the quotient functor.

Let $M \in \mathcal{T}$ such that $\mathcal{T} / \mathcal{I}=\langle F(M)\rangle_{\mathcal{T} / \mathcal{I}, r+1}$. Up to isomorphism, we can assume $M$ is finitely generated and projective as a $B$-module. Let $\mathcal{F}$ be the sheaf over $\mathrm{P}^{n-1}$ corresponding to the graded $B$-module $M$. The differential on $M$ gives a map $d: \mathcal{F} \rightarrow \mathcal{F}(1)$. Let $\mathcal{G}=$ ker $d(1) /$ im $d$. Pick $x$ a closed point of $\mathbf{P}^{n-1}$ such that $\mathcal{G}_{x}$ is a projective $\mathcal{O}_{x}$-module. Then, there is a projective $\mathcal{O}_{x}$-module $R$ such that $\operatorname{ker} d_{x}=\operatorname{im} d_{x} \oplus R$. We have an exact sequence $0 \rightarrow R \rightarrow \mathcal{F}_{x} \rightarrow \mathcal{F}_{x} / R \rightarrow 0$ of differential $\mathcal{O}_{x}$-modules. Since $\mathcal{F}_{x} / R$ is acyclic, it follows that $R \rightarrow \mathcal{F}_{x}$ is an isomorphism in $\operatorname{Ddiff}\left(\mathcal{O}_{x}\right)$, the derived category of differential $\mathcal{O}_{x}$-modules. Let $I(x)$ be the prime ideal of $B$ corresponding to the line $x$ of $\mathbf{A}^{n}$. Note that the differential graded $B$-module $B / I(x)$ (the differential is 0 ) is in $\mathcal{T}$. So, $F(B / I(x)) \in\langle F(M)\rangle_{\mathcal{T} / \mathcal{I}, r+1}$, hence 
$k_{x} \in\left\langle\mathcal{F}_{x}\right\rangle_{D \operatorname{diff}\left(\mathcal{O}_{x}\right), r+1}$, hence $k_{x} \in\left\langle\mathcal{O}_{x}\right\rangle_{D \operatorname{diff}\left(\mathcal{O}_{x}\right), r+1}$. By Proposition 7.14, we get $r \geq n-1$. Hence, $\operatorname{dim} A$-stab $\geq n-1=\operatorname{ll}(A)-2$. Now, Proposition 8.6 gives the conclusion.

The proof of the inequality $\operatorname{dim} D^{b}\left(\Lambda\left(k^{n}\right)\right.$-mod) $\geq n$ is similar (and easier). Proposition 7.38 gives the inequality $\operatorname{dim} D^{b}\left(\Lambda\left(k^{n}\right)-\bmod \right) \leq n$.

8.2.4. We assume here that $k$ is a field of characteristic $p>0$.

Proposition 8.13. Let $G$ be a finite group and $B$ a block of $k G$. Let $D$ be a defect group of $B$. Then, $\operatorname{dim} B$-stab $=\operatorname{dim}(k D)$-stab and $\operatorname{dim} D^{b}(B$-mod $)=\operatorname{dim} D^{b}((k D)$-mod $)$

Proof. Recall that a defect group $D$ of $B$ is a (smallest) subgroup such that the identity functor of $B$-mod is a direct summand of $\operatorname{Ind}_{D}^{G} \operatorname{Res}_{D}^{G}$. Since $k D$ is a direct summand of $B$ as a $(k D, k D)$ bimodule, the Proposition follows from Lemma 3.3 .

Given $P$ a finite $p$-group and $Q$ a maximal subgroup of $P$, we denote by $\beta_{Q} \in H^{2}(P, \mathbf{Z} / p)$ the class of the exact sequence

$$
0 \rightarrow \mathbf{Z} / p \rightarrow \operatorname{Ind}_{Q}^{P} \mathbf{Z} / p \stackrel{x-1}{\longrightarrow} \operatorname{Ind}_{Q}^{P} \mathbf{Z} / p \rightarrow \mathbf{Z} / p \rightarrow 0
$$

where $x \in P-Q$.

The following Proposition gives a recursive bound for the dimension of the stable category.

Proposition 8.14. Let $G$ be a finite group and $B$ a block of $k G$. Let $D$ be a defect group of $B$. Let $D_{1}, \ldots, D_{n}$ be a family of maximal subgroups of $D$ such that $\beta_{D_{1}} \cdots \beta_{D_{n}}=0$ (such a family exists and one can assume $\left.n \leq \frac{p+1}{p^{2}}|D: \Phi(D)|\right)$.

Then, $\operatorname{dim} B$-stab $<2 \sum_{i}\left(1+\operatorname{dim} k D_{i}\right.$-stab $)$ and $\operatorname{dim} D^{b}(B$-mod $)<2 \sum_{i}\left(1+\operatorname{dim} D^{b}\left(k D_{i}\right.\right.$-mod $\left.)\right)$.

Proof. By Proposition 8.13, it is enough to consider the case where $G=D$. Then, [Ca, Lemma 3.9] asserts that there is a $k G$-module $M$ which has $k$ as a direct summand and has a filtration $0=M_{0} \subset M_{1} \subset \cdots \subset M_{2 n}=M$ with $M_{2 i-1} / M_{2 i-2} \simeq \operatorname{Ind}_{D_{i}}^{G} \Omega^{t_{i}} k$ and $M_{2 i} / M_{2 i-1} \simeq \operatorname{Ind}_{D_{i}}^{G} \Omega^{t_{i}^{\prime}} k$ for some integers $t_{i}, t_{i}^{\prime}$. We conclude as in $\S 3.2 .2$.

The existence of the family is Serre's Theorem on product of Bockstein's, cf e.g. Ben, Theorem 7.4.3].

Theorem 8.15. Let $G$ be a finite group, $B$ a block of $k G$ over a field $k$ of characteristic 2 . Let $D$ be a defect group of $B$. Then, $\operatorname{repdim} B \geq 2+\operatorname{dim} B$-stab $>r$ and $\operatorname{dim} D^{b}(B$-mod $) \geq r$, where $r$ is the 2-rank of $D$.

Proof. The first inequality is given by Proposition 8.6. By Proposition 8.13, it suffices to prove the Theorem for $G=D$ and $B=k D$. Let $P$ be an elementary abelian 2-subgroup of $D$ with rank the 2 -rank of $D$. Then, $\operatorname{dim} k P$-stab $\leq \operatorname{dim} k D$-stab by Lemma 3.3. Now, $k P \simeq \Lambda\left(k^{r}\right)$ and the Theorem follows from Theorem 8.12. The derived category assertion has a similar proof.

Let us recall a conjecture of D. Benson :

Conjecture 8.16 (Benson). Let $G$ be a finite group, $B$ a block of $k G$ over a field $k$ of characteristic $p$. Then, $\operatorname{ll}(B)>p-\operatorname{rank}(D)$.

From Theorem 8.15 and Proposition 8.6, we deduce :

Theorem 8.17. Benson's conjecture 8.10 holds for $p=2$. 


\section{REFERENCES}

[Au M. Auslander, Representation dimension of Artin algebras, Queen Mary College Mathematics Notes, London, 1971.

[Ba] J. Backelin, On the rate of growth of the homologies of Veronese subrings, Lecture Notes in Math. 1183, 79-100, Springer Verlag, 1986.

[Bei] A.A. Beilinson, Coherent sheaves on $\mathbf{P}^{n}$ and problems of linear algebra, Funct. Anal. Appl. 12 (1978), 214-216.

[Bel] A. Beligiannis, Relative homological algebra and purity in triangulated categories, J. Alg. 227 (2000), 268-361.

[Ben] D.J. Benson, "Representations and cohomology", vol. II, Cambridge University Press, 1991.

[BeCaRi] D.J. Benson, J.F. Carlson and J. Rickard, Thick subcategories of the stable module category, Fundamenta Mathematicae 153 (1997), 59-80

[BöNee] M. Bökstedt and A. Neeman, Homotopy limits in triangulated categories, Compositio Math. 86 (1993), 209-234.

[BoVdB] A. Bondal and M. Van den Bergh, Generators and representability of functors in commutative and noncommutative geometry, Moscow Math. J. 3 (2003), 1-36.

[Ca] J.F. Carlson, Cohomology and induction from elementary abelian subgroups, Q. J. Math. 51 (2000), 169-181.

[ChKeNee] J.D. Christensen, B. Keller and A. Neeman, Failure of Brown representability in derived categories, Topology 40 (2001), 1339-1361.

[CPS] E. Cline, B. Parshall and L. Scott, Finite dimensional algebras and highest weight categories, J. reine angew. Math. 391 (1988), 85-99.

[ErHoIySc] K. Erdmann, T. Holm, O. Iyama and J. Schröer, Radical embeddings and representation dimension, Adv. Math. 185 (2004), 159-177.

[GeMa] S.I. Gelfand and Yu.I. Manin, Homological Algebra, Springer Verlag, 1999.

[Ho] T. Holm, Representation dimension of some tame blocks of finite groups, Alg. Colloq. 10 (2003), 275-284.

[IgTo] K. Igusa and G. Todorov, On the finistic global dimension conjecture for Artin algebras, preprint, 2002 .

[Iy] O. Iyama, Finiteness of representation dimension, Proc. Amer. Math. Soc. 131 (2003), $1011-1014$.

[Kap] M. Kapranov, On the derived category of coherent sheaves on some homogeneous spaces, Inv. Math. 92 (1988), 479-508.

[Kaw] Y. Kawamata, Equivalences of derived categories of smooth stacks, preprint math.AG/0210439.

[Ke] B. Keller, Deriving DG Categories, Ann. Sci. Ec. Norm. Sup. 27 (1994), 63-102.

[KeVo] B. Keller and D. Vossieck, Sous les catégories dérivées, C. R. Acad. Sci. Paris, Série I, 305 (1987), 225-228.

[Ma] H. Matsumura, Commutative Algebra, second edition, Benjamin Cummings, 1980.

[Nee1] A. Neeman, The chromatic tower for $D(R)$, Topology 31 (1992), 519-532.

[Nee2] A. Neeman, The connection between the K-theory localization theorem of Thomason, Trobaugh and Yao and the smashing subcategories of Bousfield and Ravenel, Ann. Sci. Éc. Norm. Sup. 25 (1992), 546-566.

[Nee3] A. Neeman, The Grothendieck duality theorem via Bousfield's techniques and Brown representability, J. Amer. Math. Soc. 9 (1996), 205-236.

[Nee4] A. Neeman, On a Theorem of Brown and Adams, Topology 36 (1997), 619-645.

[Nee5] A. Neeman, "Triangulated categories", Princeton University Press, 2001.

[Or] D. Orlov, Triangulated categories of singularities and D-branes in Laudau-Ginzburg models, preprint math.AG/0302304.

[Ri] J. Rickard, Derived categories and stable equivalence, J. Pure and Appl. Alg 61 (1989), 303-317.

[SGA6] P. Berthelot, A. Grothendieck and L. Illusie, "Théorie des intersections et théorème de RiemannRoch", Lecture Notes in Mathematics 225, Springer Verlag, 1971.

[Ste] B. Stenström, "Rings of quotients", Springer Verlag, 1975. 
[Th] R.W. Thomason, The classification of triangulated subcategories, Compositio Math. 105 (1997), 1-27.

[ThTr] R.W. Thomason and T.F. Trobaugh, Higher algebraic K-theory of schemes and of derived categories, in "The Grothendieck Festschrift", vol. III, 247-435, Birkhauser, 1990.

[Xi] C. Xi, Representation dimension and quasi-hereditary algebras, Adv. in Math. 168 (2002), $193-212$. 